\title{
GAMMA SPACES AND INFORMATION
}

\author{
MATILDE MARCOLLI
}

\begin{abstract}
We investigate the role of Segal's Gamma-spaces in the context of classical and quantum information, based on categories of finite probabilities with stochastic maps and density matrices with quantum channels. The information loss functional extends to the setting of probabilistic Gamma-spaces considered here. The Segal construction of connective spectra from Gamma-spaces can be used in this setting to obtain spectra associated to certain categories of gapped systems.
\end{abstract}

\section{Contents}

1. Introduction

2. From pointed sets to probabilities

3. Information loss 11

4. Gamma spaces and finite probabilities [15]

5. Stochastic Gamma spaces 21

6. Information loss on probabilistic categories

7. Information loss and probabilistic Gamma spaces 28

8. Quantum Information and Categories 32

9. Quantum Information and Gamma Spaces 37

10. Gamma spaces, spectra, and gapped systems 41

References

\section{INTRODUCTION}

Segal introduced $\Gamma$-spaces in [30], as a way of constructing connective spectra from the data of a category $\mathcal{C}$ with a zero object and a categorical sum. A $\Gamma$-space is a functor $F_{\mathcal{C}}: \Gamma^{0} \rightarrow \Delta_{*}$ from the category of pointed finite sets to the category of pointed simplicial sets. Spectra associated to $\Gamma$-spaces are obtained by extending the functor to an endofunctor of $\Delta_{*}$ and applying it to the spheres $S^{n}$, so that one obtains a spectrum given by the sequence of spaces $X_{n}=F_{\mathcal{C}}\left(S^{n}\right)$ with structure maps $S^{1} \wedge F_{\mathcal{C}}\left(S^{n}\right) \rightarrow F_{\mathcal{C}}\left(S^{1} \wedge S^{n}\right)=X_{n+1}$. In particular, when $F_{\mathcal{C}}: \Gamma_{0} \hookrightarrow \Delta_{*}$ is the natural inclusion, that is, the $\Gamma$-space associated to the category $\mathcal{C}=\Gamma^{0}$ itself, one obtains the sphere spectrum. More generally, it is known that all connective spectra can in fact be obtained in this way, [4], [30], [33]. Moreover, $\Gamma$-spaces provide a very transparent description of the smash product of spectra, [25].

Date: 2018. 
Throughout this paper we will use equivalently the $\Gamma$ notation of Segal for the category of finite pointed sets as well as the notation $\mathcal{S}_{*}$. We trust that this will not be a source of confusion.

The main purpose of this paper is developing probabilistic versions of the Segal construction, in the setting of classical and quantum information. In the classical setting, we replace the usual category of finite pointed sets used in homotopy theoretic construction with a probabilistic version. Heuristically this corresponds to finite sets where each of the points can be the base point with certain assigned probabilities. The more precise formulation is in terms of a form of wreath product between the category of finite pointed sets and the category of finite probabilities with stochastic maps. More generally, one can start with any category $\mathcal{C}$ with a zero object and a categorical sum (coproduct) and form a similar wreath product with the category $\mathcal{F} \mathcal{P}$ of finite probabilities. The resulting category $\mathcal{P C}$ still has a zero object and a coproduct, where on the probabilities the coproduct is a product of statistically independent distributions. The Segal construction [30] of $\Gamma$-spaces from categories with zero object and sum can then be applied to the categories $\mathcal{P C}$ leading to associated spectra. For the purpose of obtaining an explicit description in the particular case where $\mathcal{C}$ is the category $\mathcal{S}_{*}$ of finite pointed sets, we reformulate the usual notion of $\Gamma$-spaces using the category of cubical sets $\square_{*}$ instead of simplicial sets. We then analyze the explicit form of the $\Gamma$-space $F_{\mathcal{P S}}: \Gamma^{0} \rightarrow \square_{*}$. We then show that the notion of $\Gamma$-space itself can be made probabilistic, by considering functors $F: \mathcal{P} \mathcal{S}_{*} \rightarrow \mathcal{P} \square_{*}$ between the corresponding probabilistic categories. These map a probabilistic pointed set $\Lambda X$ to a probabilistic pointed cubical set given by a $\Lambda$-convex combination of the cubical sets associated to the pointed sets in $\Lambda X$ by the original (non-probabilistic) $\Gamma$-space.

An information loss functional of the kind considered in [2] can be defined on the category of finite probabilities $\mathcal{F P}$ and extended to the categories $\mathcal{P} \mathcal{S}_{*}$ and $\mathcal{P} \square_{*}$ of probabilistic pointed sets and probabilistic pointed cubical sets. As in the case of [2] these information loss functionals are determined by a set of Khinchin-type axioms and are always expressible in terms of a difference of Shannon entropies and of topological invariants of cubical (or simplicial) sets.

We consider then a quantum information version $\mathcal{Q C}$ of the probabilistic categories $\mathcal{P C}$, again starting with a category $\mathcal{C}$ with zero object and sum. In this case we consider a category $\mathcal{F} \mathcal{Q}$ of finite quantum probabilities (density matrices) with morphisms given by quantum channels. The objects of the category $\mathcal{Q C}$ are collections of pairs of objects in $\mathcal{C}$ with an assigned amount of quantum interference (coherence) specified by the entries of a density matrix. A similar version $\mathcal{Q} \mathcal{A C}$ uses a category $\mathcal{A C}$ of arrows of $\mathcal{C}$ with associated density matrices. The category $\mathcal{Q C}$ still has a zero object and coproduct, hence one can again apply the Segal construction and obtain $\Gamma$-spaces (as well as corresponding probabilistic $\Gamma$-spaces) and associated spectra. In the case where the underlying deterministic category is $\mathcal{C}=\mathcal{S}_{*}$ the category of finite pointed sets, one can again describe explicitly the resulting topological spaces, from which one can see directly that they are topologically more complex than their counterparts in the case of classical probabilities. 
Finally, we consider a category $\mathcal{F} \mathcal{Q}^{\Delta}$ of gapped systems related by gap preserving quantum channels, and corresponding categories $\mathcal{Q C}^{\Delta}$ realized as subcategories of $\mathcal{Q C}$. We compute explicltly the associated $\Gamma$-space in the case of $\mathcal{C}=\mathcal{S}_{*}$. These $\Gamma$-spaces provide a natural construction of connective spectra associated to gapped systems.

In a broader perspective, we regard this as a first step in the direction of developing a probabilistic version of homotopy theory. There are several reasons why this would be a desirable goal. Mainly, one can think of the following sources of motivation:

- Physics: as mentioned above spectra and other homotopy theoretic methods are widely regarded as an important approach to study gapped systems and topological phases of matter, [15], [36]. Because of the quantum information formulation of the problem, it is expected that a development of homotopy theoretic methods that incorporate classical and quantum probabilities will be a useful tool in this setting.

- Statistics: data analysis has incorporated topological methods in the form of persistent topology and persistent homology, which are based on simplicial sets associated to a collection of data points, 6]. A version of simplicial sets and homotopy theoretic methods that incorporate probabilities may be useful in developing better categorical structures in persistent topology (model categories, etc.). This question was suggested by Jack Morava.

- Neuroscience: the idea of enriching algebraic structures with probabilities is not new, and was in fact developed in great generality in [16] and found a wide range of applications, including "pattern theory" in models of computer vision and neuroscience. The use of simplicial and homotopy theoretic methods in neuroscience is also advocated in [7], 26] and one expects that an adequate treatment will have to include probabilities along with combinatorics, [27].

While the present paper only focuses on one particular aspect, namely probabilistic versions of the Segal construction of spectra through $\Gamma$-spaces, and does not touch upon these broader motivations and applications, one should regard the purpose of this investigation within this more general context.

\section{From pointed Sets to probabilities}

The category of pointed finite sets is a category with a zero object and a categorical sum, hence one can apply to it the construction of [30] that produces a $\Gamma$-space and a spectrum, which in this case is the sphere spectrum $\mathbb{S}=\left\{S^{n}\right\}$, a result known as the Barratt-Priddy-Quillen theorem.

The main heuristic observation that we want to formalize in this paper is the fact that the Segal notion of $\Gamma$-spaces relies crucially on the construction of a category of summing functors (which we will review briefly). These behave very much like measures, so that one is lead to believe that the use of pointed sets in the construction should in fact be seen as a proxy for a measure theoretic setting. The first step in making this heuristic observation more rigorous is to consider generalizations of the 
category of pointed sets based on finite probability spaces. The main idea is to think of pointed sets as a special case where the probability is a delta function supported at the base point and morphisms are measure preserving maps (hence pointed maps).

The category of finite sets with probability measures considered in [2], 3] with measure preserving morphisms satisfying (3.3) is not directly suitable for the application of the Segal construction of [30]. Thus, we consider here some possible modifications, in the form of categories of finite sets with probability distributions and with stochastic maps.

2.1. Finite probabilities and stochastic map. We consider a category of finite probability spaces and stochastic matrices defined as follows.

Definition 2.1. Let $\mathcal{F P}$ be the category whose objects are pairs $(X, P)$ of a finite set $X$ with a probability measure $P$. Morphisms

$$
S \in \operatorname{Mor}_{\mathcal{F P}}((X, P),(Y, Q))
$$

are stochastic $(\# Y \times \# X)$-matrices $S$, with the following properties:

(1) $S_{y x} \geq 0$, for all $x \in X, y \in Y$;

(2) $\sum_{y \in Y} S_{y x}=1$ for all $x \in X$;

(3) the probability measures are related by $Q=S P$.

Remark 2.2. This category of stochastic matrices is not the same as the category FinStoch of [13], where objects are finite sets without measures, so that the third condition of Definition 2.1 above is not required. However, $\mathcal{F P}$ is the under category $1 /$ FinStoch, as discussed after Definition 3 of [3].

Note that to a stochastic matrix $S$ as above we can associate a multivalued function $f_{S}: X \rightarrow Y$ with $f_{S}\left(x_{j}\right)=\left\{y_{i} \in Y: S_{i j}>0\right\}$. The morphisms are measure preserving in the sense that the relation

$$
Q_{y}=\sum_{x \in X} S_{y x} P_{x}
$$

holds, replacing the original (3.3). The category of finite probability measures considered in [2] is a subcategory of $\mathcal{F P}$ consisting of those morphisms where, for each $x$ there is a unique $y=y(x)$ such that $S_{y x}>0$. In this case, by the stochastic condition this value must be $S_{y x}=1$ hence $S$ corresponds to a (single valued) function $f: X \rightarrow Y$ satisfying (2.1), which in this case becomes the same as (3.3),

$$
Q_{y}=\sum_{x \in f^{-1}(y)} P_{x}
$$

The sets of morphisms $\operatorname{Mor}_{\mathcal{F P}}((X, P),(Y, Q))$ are convex sets.

Remark 2.3. The usual category of finite pointed sets can be seen as the subcategory of $\mathcal{F P}$ given by elements of the form $\left(X, \delta_{x_{0}}\right)$ and morphisms given by stochastic maps $S:\left(X, \delta_{x_{0}}\right) \rightarrow\left(Y, \delta_{y_{0}}\right)$ of the form $S_{y x}=\chi_{f^{-1}(y)}(x)$, with $\chi$ the indicator function, so that $\delta_{y_{0}, y}=\sum_{x} S_{y x} \delta_{x_{0}, x}=\sum_{x \in f^{-1}(y)} \delta_{x_{0}, x}$. 
Remark 2.4. The objects $(X, P)$ of the category $\mathcal{F P}$ can be thought of as fuzzy sets, with $P_{x}$ the value at the point $x \in X$ of the membership function of the fuzzy set, [37].

2.1.1. Zero object. The singletons in the category of finite probabilities are zero objects.

Lemma 2.5. The category $\mathcal{F P}$ has zero objects given by singleton sets $(\{x\}, 1)$.

Proof. For all objects $(Y, Q)$ there is a unique morphism $\hat{Q}:(\{x\}, 1) \rightarrow(Y, Q)$ which is given by $\hat{Q}_{y x}=Q_{y}$, and a unique morphism $\hat{1}:(Y, Q) \rightarrow(\{x\}, 1)$ given by $\hat{1}_{x y}=1$ for all $y \in Y$, so that $1=\sum_{y \in Y} Q_{y}=\sum_{y \in Y} \hat{1}_{x y} Q_{y}$. Thus, $(\{x\}, 1)$ is a zero-object.

We will use the notation $\hat{1}_{(X, P)}$ for the unique morphism $\hat{1}_{(X, P)}:(X, P) \rightarrow(\{x\}, 1)$, whenever it is useful to keep track explicitly of the source object $(X, P)$.

2.1.2. The target morphism. Given any pair of objects $(X, P)$ and $(Y, Q)$ in $\mathcal{F P}$, there is always a distinguished morphism, which we denote by $\hat{Q}:(X, P) \rightarrow(Y, Q)$, which is defined by $\hat{Q}_{b a}=Q_{b}$. This clearly satisfies $Q_{b}=\sum_{a} \hat{Q}_{b a} P_{a}$. We refer to this morphism as "the target morphism".

Lemma 2.6. The target morphism $\hat{Q}:(X, P) \rightarrow(Y, Q)$ has the property that, given any morphism $S:\left(X^{\prime}, P^{\prime}\right) \rightarrow(X, P)$ and any morphism $S^{\prime}:(Y, Q) \rightarrow\left(Y^{\prime}, Q^{\prime}\right)$ the compositions satisfy $\hat{Q} \circ S=\hat{Q}$ and $S^{\prime} \circ \hat{Q}=\hat{Q}^{\prime}$.

Proof. We have $(\hat{Q} \circ S)_{b a^{\prime}}=\sum_{a} \hat{Q}_{b a} S_{a a^{\prime}}=Q_{b} \sum_{a} S_{a a^{\prime}}=Q_{b}$ for all $a^{\prime} \in X^{\prime}$, and $\left(S^{\prime} \circ \hat{Q}\right)_{b^{\prime} a}=\sum_{a^{\prime}} S_{b^{\prime} a^{\prime}}^{\prime} Q_{a^{\prime}}=Q_{b^{\prime}}^{\prime}$ for all $a \in X$.

Remark 2.7. The target morphisms are the categorical zero morphisms, that is, the morphisms that factor through the zero object.

2.2. Coproduct of finite probabilities. We want to construct a coproduct of finite probabilities which reduces to the coproduct of pointed sets in the case where the measures are delta measures.

2.2.1. Finite probabilities as combinations of pointed sets. We can equivalently regard a finite probability $(X, P)$ as a finite set $X$ where each point $x \in X$ can be chosen as the base point with probability $P_{x}$. Thus, we can regard the object $(X, P)$ as a formal convex combination of pointed sets,

$$
(X, P)=\sum_{x \in X} P_{x}(X, x) .
$$

This interpretation means that we can embed the category $\mathcal{F P}$ of finite probabilities in a category $\mathcal{P} \mathcal{S}_{*}$ of probabilistic pointed sets defined as follows. 
Definition 2.8. The category $\mathcal{P} \mathcal{S}_{*}$ of probabilistic pointed sets has objects that are convex combinations of pointed sets

$$
\Lambda X=\sum_{i} \lambda_{i}\left(X_{i}, x_{i}\right)
$$

where $\Lambda=\left(\lambda_{i}\right)$ wtih $\lambda_{i} \geq 0$ and $\sum_{i} \lambda_{i}=1$ and $X=\left\{\left(X_{i}, x_{i}\right)\right\}$ a finite collection of pointed sets. The morphisms in $\mathcal{P S}_{*}$ are given by $\Phi \in \operatorname{Mor}_{\mathcal{P} \mathcal{S}_{*}}\left(\Lambda X, \Lambda^{\prime} X^{\prime}\right)$ consisting of a pair $\Phi=(S, F)$

(1) $S$ is a stochastic map with $S \Lambda=\Lambda^{\prime}$

(2) $F=\left(F_{j i}\right)$ is a collection of probabilistic pointed maps $F_{j i}:\left(X_{i}, x_{i}\right) \rightarrow\left(X_{j}^{\prime}, x_{j}^{\prime}\right)$. Here a probabilistic pointed map $F_{j i}$ is a finite set $\left\{F_{j i, a}\right\}$ of pointed maps $F_{j i, a}$ : $\left(X_{i}, x_{i}\right) \rightarrow\left(X_{j}^{\prime}, x_{j}^{\prime}\right)$ together with a set of probabilities $\mu_{a}^{(j i)}$ with $\sum_{a} \mu_{a}^{(j i)}=S_{j i}$.

Thus we regard a morphism between two probabilistic pointed sets as a collection of probabilistic pointed maps $F=\left\{F_{j i, a}\right\}$. This means that for a fixed source $\left(X_{i}, x_{i}\right)$ and a point $x \in\left(X_{i}, x_{i}\right)$ the value $F(x)$ is obtained by first choosing a map $F_{j i, a}$ with probability $\mu_{a}^{(j i)}$. Equivalently, one chooses the set $F_{j i}=\left\{F_{j i, a}\right\}$ with probability $S_{j i}$, which is the sum of all the probabilities $\mu_{a}^{(j i)}$ of choosing one of the maps $F_{j i, a}$ in the set. This includes the case where $F_{j i}$ consists of a single pointed map applied with probability $S_{j i}$.

Remark 2.9. The composition of two morphisms $\Phi=(S, F): \Lambda X \rightarrow \Lambda^{\prime} X^{\prime}$ and $\Phi^{\prime}=$ $\left(S^{\prime}, F^{\prime}\right): \Lambda^{\prime} X^{\prime} \rightarrow \Sigma Y$ is given by $\Phi^{\prime} \circ \Phi=\left(S^{\prime} \circ S, F^{\prime} \circ F\right)$, where $S^{\prime} \circ S$ is the product of the stochastic matrices and $F^{\prime} \circ F=\left\{\left(F^{\prime} \circ F\right)_{k i}\right\}$ with the set $\left(F^{\prime} \circ F\right)_{k i}=\left\{F_{k j, a}^{\prime} \circ F_{j i, b}\right\}$ with probabilities $\mu_{a}^{(k j)} \mu_{b}^{(j i)}$ with $\sum_{a, b, j} \mu_{a}^{(k j)} \mu_{b}^{(j i)}=\sum_{j} S_{k j}^{\prime} S_{j i}=\left(S^{\prime} \circ S\right)_{k i}$, so that the probability associated to the set $\left(F^{\prime} \circ F\right)_{k i}$ in $F^{\prime} \circ F$ is $\left(S^{\prime} \circ S\right)_{k i}$.

Remark 2.10. An embedding of the category $\mathcal{F} \mathcal{P}$ in the category $\mathcal{P} \mathcal{S}_{*}$ is obtained by mapping $\Lambda=\left(\lambda_{i}\right)$ to the set $\Lambda \star=\sum_{i} \lambda_{i}\left(\left\{\star_{i}\right\}, \star_{i}\right)$. and morphisms $S \Lambda=\Lambda^{\prime}$ to $\Phi=(S, \mathbf{1})$ with $\mathbf{1}=\left\{1_{j i}\right\}$ with probabilities $S_{j i}$.

Remark 2.11. There is a forgetful functor from $\mathcal{P} \mathcal{S}_{*}$ to $\mathcal{F P}$ that maps $\Lambda X$ to the finite probability $\Lambda$ and a morphism $\Phi=(S, F)$ to the stochastic matrix $S$.

2.2.2. Zero objects. The category $\mathcal{P} \mathcal{S}_{*}$ of probabilistic pointes sets also has zero objects given by singletons.

Lemma 2.12. The objects $\Lambda X$ given by a singleton set $X=(\{x\}, x)$ and $\Lambda=1$ are zero objects in $\mathcal{P} \mathcal{S}_{*}$.

Proof. Given any object $\Lambda X=\sum_{i} \lambda_{i}\left(X_{i}, x_{i}\right)$, there is a unique morphism $\Phi=(S, F)$ : $(\{x\}, x) \rightarrow \sum_{i} \lambda_{i}\left(X_{i}, x_{i}\right)$ with $S=\hat{\Lambda}$, the unique morphism $\hat{\Lambda}$ from the zero-obejct $(\{x\}, 1)$ of $\mathcal{F} \mathcal{P}$ to the finite probability $\Lambda$ and with $F=\left(F_{i}\right)$ with $F_{i}: x \mapsto x_{i}$ with probability $\lambda_{i}$. Moreover, there is also a unique morphism $\Phi=(S, F): \Lambda X \rightarrow$ 
$(\{x\}, x)$ where $S=\hat{1}_{\Lambda}$ is the unique morphism in $\mathcal{F P}$ from the finite probability $\Lambda$ to the zero object $(\{x\}, 1)$ and $F=\left(F_{i}\right)$ with $F_{i}:\left(X_{i}, x_{i}\right) \rightarrow(\{x\}, x)$ the constant function with probability 1 .

2.2.3. Coproduct of probabilistic pointed sets. The category $\mathcal{P} \mathcal{S}_{*}$ has a coproduct inherited from the category of pointed sets.

Definition 2.13. Given $\Lambda X=\sum_{i=1}^{N}\left(X_{i}, x_{i}\right)$ and $\Lambda^{\prime} X^{\prime}=\sum_{j=1}^{M} \lambda_{j}^{\prime}\left(X_{j}^{\prime}, x_{j}^{\prime}\right)$ we have

$$
\Lambda X \amalg \Lambda^{\prime} X^{\prime}:=\sum_{i j} \lambda_{i} \lambda_{j}^{\prime}\left(X_{i}, x_{i}\right) \vee\left(Y_{j}, x_{j}^{\prime}\right),
$$

with the usual coproduct of pointed sets

$$
\left(X_{i}, x_{i}\right) \vee\left(Y_{j}, x_{j}^{\prime}\right)=\left(X_{i} \sqcup Y_{j} / x_{i} \sim y_{j}, x_{i} \sim y_{j}\right) .
$$

In the case of two probabilistic pointed sets obtained from two finite probability distributions as in (2.2), this corresponds to the intuition that one considers each point $x \in X$ as the base point with probability $P_{x}$, and similarly with $X^{\prime}$. Thus, when forming the coproduct, the probability that it is obtained by identifying $x \in X$ with $x^{\prime} \in X^{\prime}$ is the product of the probabilities $P_{x}$ and $P_{x^{\prime}}^{\prime}$, namely the probability of independently choosing $x$ and $x^{\prime}$ as the respective base points.

Lemma 2.14. The coproduct induced by (2.3) on probabilistic pointed sets obtained from finite probability distributions as in (2.2) is the product of statistically independent probabilities

$$
(X, P) \amalg\left(X^{\prime}, P^{\prime}\right)=\left(X \times X^{\prime}, P \cdot P^{\prime}\right) .
$$

Proof. In the case of two probabilistic pointed sets obtained from two finite probability distributions as in (2.2), this coproduct is given by

$$
(X, P) \amalg\left(X^{\prime}, P^{\prime}\right)=\sum_{x, x^{\prime}} P_{x} P_{x^{\prime}}^{\prime}(X, x) \vee\left(X^{\prime}, x^{\prime}\right) .
$$

We can interpret the right-hand-side of (2.4) as a probability space by applying the forgetful functor from $\mathcal{P} \mathcal{S}_{*}$ to $\mathcal{F P}$. This identifies it with the finite probability $\left(X \times X^{\prime}, P \cdot P^{\prime}\right)$.

Remark 2.15. It is preferable to work with the category $\mathcal{P} \mathcal{S}_{*}$ rather than with $\mathcal{F P}$, since just retaining the information of the finite probability would give the same product probability space for any underlying binary operation on probabilistic pointed sets, without remembering the specific operation on the underlying sets. 
2.2.4. Statistical independence: product or coproduct? It is well known that the category of measure spaces or of finite probability spaces with measure preserving map (hence also the category $\mathcal{F P}$ considered here) does not have a universal categorical product, that is, an object $X_{1} \times X_{2}$ such that, for all morphisms $f_{1}: X \rightarrow X_{1}$ and $f_{2}: X \rightarrow X_{2}$ there exists a unique morphism $h: X \rightarrow X_{1} \times X_{2}$ such that the diagram commutes

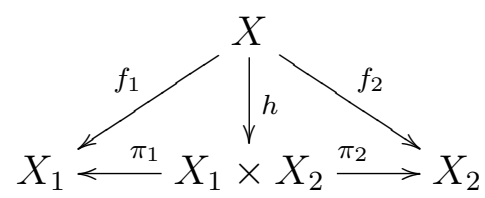

However, as shown in [11], the category of finite probability measures with measure preserving maps is a "tensor category with projections", namely a tensor category $(\mathcal{C}, \otimes)$ together with two natural transformations $\pi_{i}: \otimes \rightarrow \Pi_{i}$ where $\Pi_{i}\left(X_{1}, X_{2}\right)=X_{i}$, such that for any pairs of morphisms $f_{i}: Y_{i} \rightarrow X_{i}$, the diagram commutes:

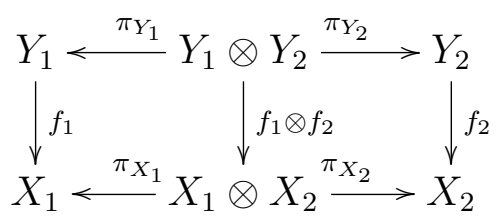

In a tensor category with projections two morphisms $f_{i}: X \rightarrow X_{i}$ are independent if there exists a morphism $h: X \rightarrow X_{1} \otimes X_{2}$ such that the product diagram (2.6) commutes. In the category of finite probabilities with measure preserving maps this notion of independence agrees with the usual notion of stochastic independence, with

$$
\left(X_{1}, P_{1}\right) \otimes\left(X_{2}, P_{2}\right)=\left(X_{1} \times X_{2}, P_{1} P_{2}\right)
$$

the product of independent probability spaces.

Tensor categories with projections (semicartesian monoidal categories) have the property that projections are unique when they exist, and their existence is equivalent to the terminality of the unit, [23].

The point of view discussed here shows that the product of statistically independent measures may be interpreted as a coproduct instead of a product on the category $\mathcal{F} \mathcal{P}$ of finite measures.

2.3. Universal property of the coproduct of probabilistic pointed sets. The coproduct of Definition 2.13 in the category $\mathcal{P} \mathcal{S}_{*}$ satisfies the universal property.

Definition 2.16. Given objects $\Lambda X=\sum_{a} \lambda_{a}\left(X_{a}, x_{a}\right)$ and $\Sigma Y=\sum_{k} \sigma_{k}\left(Y_{k}, y_{k}\right)$ and morphisms $\Phi=(S, F): \Lambda X \rightarrow \Sigma Y$ and $\Phi^{\prime}=\left(S^{\prime}, F^{\prime}\right): \Lambda^{\prime} X^{\prime} \rightarrow \Sigma Y$ with $S \Lambda=$ $\Sigma$ and $S^{\prime} \Lambda^{\prime}=\Sigma$ and with $F=\left\{f_{k a, r}\right\}_{r=1}^{N}$ with probabilities $\sum_{r} \mu_{r}^{(k a)}=S_{k a}$ and $F^{\prime}=\left\{f_{k a^{\prime}, r^{\prime}}^{\prime}\right\}_{r^{\prime}=1}^{M}$ with probabilities $\sum_{r^{\prime}} \mu_{r^{\prime}}^{(k a)}=S_{k a}^{\prime}$ we define $F \vee F^{\prime}$ as the collection $\left\{f_{k a, r} \vee f_{k a^{\prime}, r^{\prime}}^{\prime}\right\}$ of pointed maps from the coproducts of pointed sets with probabilities $\sigma_{k}^{-1} \mu_{r}^{(k a)} \mu_{r^{\prime}}^{\left(k a^{\prime}\right)}$ for $\sigma_{k} \neq 0$ and $M^{-1} \mu_{r}^{(k a)}+N^{-1} \mu_{r^{\prime}}^{\left(k a^{\prime}\right)}$ for $\sigma_{k}=0$. 
Theorem 2.17. For any objects $\Lambda X$ and $\Lambda^{\prime} X^{\prime}$ in $\mathcal{P} \mathcal{S}_{*}$, there are unique morphisms $\Psi: \Lambda X \rightarrow \Lambda X \amalg \Lambda^{\prime} X^{\prime}$ and $\Psi^{\prime}: \Lambda^{\prime} X^{\prime} \rightarrow \Lambda X \amalg \Lambda^{\prime} X^{\prime}$ such that, for any object $\Sigma Y$, with $\Sigma=\left(\sigma_{k}\right)$, in $\mathcal{P} \mathcal{S}_{*}$ and any choice of morphisms $\Phi=(S, F): \Lambda X \rightarrow \Sigma Y$ and $\Phi^{\prime}=\left(S^{\prime}, F^{\prime}\right): \Lambda^{\prime} X^{\prime} \rightarrow \Sigma Y$, there is a unique morphism $\Phi \amalg \Phi^{\prime}: \Lambda X \amalg \Lambda^{\prime} X^{\prime} \rightarrow \Sigma Y$ such that the diagram commutes

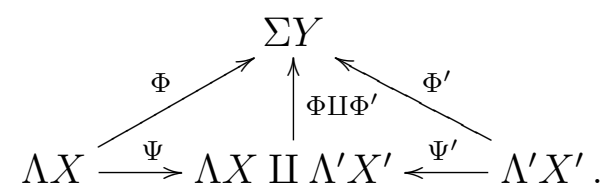

where $\left(\Phi \amalg_{\lambda} \Phi^{\prime}\right)=\left(S \amalg S^{\prime}, F \vee F^{\prime}\right)$ with

$$
\left(S \amalg_{\lambda} S^{\prime}\right)_{k,\left(a, a^{\prime}\right)}= \begin{cases}\sigma_{k}^{-1} \cdot S_{k, a} \cdot S_{k, a^{\prime}}^{\prime} & \sigma_{k} \neq 0 \\ S_{k, a}+S_{k, a^{\prime}}^{\prime} & \sigma_{k}=0 .\end{cases}
$$

and with $F \vee F^{\prime}$ as in Definition 2.16.

Proof. We have $\Psi=(\mathcal{I}, \mathcal{F})$ and $\Psi^{\prime}=\left(\mathcal{I}^{\prime}, \mathcal{F}^{\prime}\right)$ where the morphisms $\mathcal{I} \in \operatorname{Mor}_{\mathcal{F P}}(\Lambda, \Lambda$. $\left.\Lambda^{\prime}\right), \mathcal{I}^{\prime} \in \operatorname{Mor}_{\mathcal{F P}}\left(\Lambda^{\prime}, \Lambda \cdot \Lambda^{\prime}\right)$ are given by

$$
(\mathcal{I})_{\left(b, b^{\prime}\right), a}=\delta_{a b} \lambda_{b^{\prime}}^{\prime} \quad \text { and } \quad\left(\mathcal{I}^{\prime}\right)_{\left(b, b^{\prime}\right), a^{\prime}}=\delta_{a^{\prime} b^{\prime}} \lambda_{b}
$$

These satisfy $\mathcal{I} \Lambda=\Lambda \cdot \Lambda^{\prime}$ and $\mathcal{I}^{\prime} \Lambda^{\prime}=\Lambda \cdot \Lambda^{\prime}$. The probabilistic pointed maps $\mathcal{F}=$ $\left(\mathcal{F}_{\left(b, b^{\prime}\right), a}\right)$ and $\mathcal{F}^{\prime}=\left(\mathcal{F}_{\left(b, b^{\prime}\right), a^{\prime}}^{\prime}\right)$ are given by the standard pointed inclusion maps to the coproduct of pointed sets $\mathcal{F}_{\left(b, b^{\prime}\right), a}=\delta_{a b} \mathcal{F}_{b^{\prime} b}$ with $\mathcal{F}_{b^{\prime} b}:\left(X_{b}, x_{b}\right) \hookrightarrow\left(X_{b}, x_{b}\right) \vee\left(X_{b^{\prime}}^{\prime}, x_{b^{\prime}}^{\prime}\right)$ the inclusion map of the coproduct of pointed sets, chosen with probability $\lambda_{b^{\prime}}^{\prime}$, and similarly $\mathcal{F}_{\left(b, b^{\prime}\right), a^{\prime}}^{\prime}=\delta_{a^{\prime} b^{\prime}} \mathcal{F}_{b b^{\prime}}^{\prime}$ with and $\mathcal{F}_{b b^{\prime}}^{\prime}:\left(X_{b^{\prime}}^{\prime}, x_{b^{\prime}}^{\prime}\right) \hookrightarrow\left(X_{b}, x_{b}\right) \vee\left(X_{b^{\prime}}^{\prime}, x_{b^{\prime}}^{\prime}\right)$ the inclusions taken with probabilities $\lambda_{b}$.

A morphism $\Theta: \Lambda X \amalg \Lambda^{\prime} X^{\prime} \rightarrow \Sigma Y$ is given by $\Theta=(\tilde{S}, \tilde{F})$ where $\tilde{S} \in \operatorname{Mor}_{\mathcal{F P}}(\Lambda$. $\left.\Lambda^{\prime}, \Sigma\right)$ and $\tilde{F}=\left\{\tilde{F}_{k,(i, j)}\right\}$ is a collection of pointed maps $\tilde{F}_{k,(i, j)}=\left\{\tilde{F}_{k,(i, j), s}\right\}$ from the pointed sets $\left(X_{i}, x_{i}\right) \vee\left(X_{j}^{\prime}, x_{j}^{\prime}\right)$ to the pointed set $\left(Y_{k}, y_{k}\right)$, with probabilities $\sum_{s} \mu_{s}^{(k,(i, j))}=\tilde{S}_{k,(i, j)}$.

The compositions $\Theta \circ \Psi$ and $\Theta \circ \Psi^{\prime}$ are given on the stochastic matrices by

$$
\sum_{a, a^{\prime}} \tilde{S}_{k,\left(a, a^{\prime}\right)} \lambda_{a} \lambda_{a^{\prime}}^{\prime}=\sigma_{k}, \quad \text { for } \quad \Sigma=\left(\sigma_{k}\right)
$$

while the compositions with $\mathcal{I}$ and $\mathcal{I}^{\prime}$ are given by

$$
\begin{gathered}
\sum_{a, a^{\prime}} \tilde{S}_{k,\left(a, a^{\prime}\right)} \mathcal{I}_{\left(a, a^{\prime}\right), b}=\sum_{a, a^{\prime}} \tilde{S}_{k,\left(a, a^{\prime}\right)} \delta_{a b} \lambda_{a^{\prime}}^{\prime}=\sum_{a^{\prime}} \tilde{S}_{k,\left(b, a^{\prime}\right)} \lambda_{a^{\prime}}^{\prime} \\
\sum_{a, a^{\prime}} \tilde{S}_{k,\left(a, a^{\prime}\right)} \mathcal{I}_{\left(a, a^{\prime}\right), b^{\prime}}^{\prime}=\sum_{a} \tilde{S}_{k,\left(a, b^{\prime}\right)} \lambda_{a} .
\end{gathered}
$$

At the level of the pointed maps we have the compositions $\tilde{F} \circ \mathcal{F}$ and $\tilde{F} \circ \mathcal{F}^{\prime}$, which are compositions with the inclusions $\tilde{F} \circ \mathcal{F}=\left\{\tilde{F}_{k,(i, j), s} \circ \mathcal{F}_{j i}\right\}$ with probabilities $\mu_{s}^{(k,(i, j))} \lambda_{j}^{\prime}$ and $\tilde{F} \circ \mathcal{F}^{\prime}=\left\{\tilde{F}_{k,(i, j), s} \circ \mathcal{F}_{i j}^{\prime}\right\}$, with probabilities $\mu_{s}^{(k,(i, j))} \lambda_{i}$. 
The morphism $\tilde{S}_{k,\left(a, a^{\prime}\right)}=\sigma_{k}^{-1} S_{k, a} S_{k, a^{\prime}}^{\prime}$ for $\sigma_{k} \neq 0$ and $\tilde{S}_{k,\left(a, a^{\prime}\right)}=S_{k, a}+S_{k, a^{\prime}}^{\prime}$ when $\sigma_{k}=0$ satisfies $(2.9)$, since in the case $\sigma_{k} \neq 0$

$$
\sum_{a, a^{\prime}} \sigma_{k}^{-1} S_{k, a} S_{k, a^{\prime}}^{\prime} \lambda_{a} \lambda_{a^{\prime}}^{\prime}=\sigma_{k}^{-1} \sigma_{k}^{2}
$$

while in the case with $\sigma_{k}=0$ we have $\sum_{a, a^{\prime}}\left(S_{k, a}+S_{k, a^{\prime}}^{\prime}\right) \lambda_{a} \lambda_{a^{\prime}}^{\prime}=0$ since $\sum_{a} S_{k, a} \lambda_{a}=$ $\sum_{a^{\prime}} S_{k, a^{\prime}}^{\prime} \lambda_{a^{\prime}}^{\prime}=0$. Moreover, we have

$$
\begin{aligned}
& \sum_{a, a^{\prime}} \sigma_{k}^{-1} S_{k, a} S_{k, a^{\prime}}^{\prime} \mathcal{I}_{\left(a, a^{\prime}\right), b}=\sigma_{k}^{-1} S_{k, b} \sum_{a^{\prime}} S_{k, a^{\prime}}^{\prime} \lambda_{a^{\prime}}=S_{k, b} \quad \sigma_{k} \neq 0 \\
& \sum_{a, a^{\prime}}\left(S_{k, a}+S_{k, a^{\prime}}^{\prime}\right) \mathcal{I}_{\left(a, a^{\prime}\right), b}=S_{k, b}+\sum_{a^{\prime}} S_{k, a^{\prime}}^{\prime} \lambda_{a^{\prime}}=S_{k, b} \quad \sigma_{k}=0 .
\end{aligned}
$$

By the universal property of the coproduct of pointed sets, there is a unique map $f_{k i, r} \vee f_{k j, r^{\prime}}^{\prime}$ with the property that $\left(f_{k i, r} \vee f_{k j, r^{\prime}}^{\prime}\right) \circ \mathcal{F}_{j i}=f_{k i, r}$ and $\left(f_{k i, r} \vee f_{k j, r^{\prime}}^{\prime}\right) \circ \mathcal{F}_{i j}^{\prime}=$ $f_{k j, r^{\prime}}^{\prime}$. We consider the resulting map $F \vee F^{\prime}$ as in Definition 2.16, with the probability assigned to $f_{k i, r} \vee f_{k j, r^{\prime}}^{\prime}$ given by $\sigma_{k}^{-1} \mu_{r}^{(k i)} \mu_{r^{\prime}}^{(k j)}$ for $\sigma_{k} \neq 0$ and $\mu_{r}^{(k i)}+\mu_{r^{\prime}}^{(k j)}$ for $\sigma_{k}=0$. Then the probability associated to the composition $\left(f_{k i, r} \vee f_{k j, r^{\prime}}^{\prime}\right) \circ \mathcal{F}_{j i}$ is given by $\sigma_{k}^{-1} \mu_{r}^{(k i)} \mu_{r^{\prime}}^{(k j)} \lambda_{j}^{\prime}$ when $\sigma_{k} \neq 0$ and $\left(M^{-1} \mu_{r}^{(k i)}+N^{-1} \mu_{r^{\prime}}^{(k j)}\right) \lambda_{j}^{\prime}$ when $\sigma_{k}=0$. Since for all $j, r^{\prime}$ the composition is equal to $f_{k i, r}$ the probabilities correspondingly should add up. Indeed, we have

$$
\sum_{j, r^{\prime}} \sigma_{k}^{-1} \mu_{r}^{(k i)} \mu_{r^{\prime}}^{(k j)} \lambda_{j}^{\prime}=\mu_{r}^{(k i)} \sigma_{k}^{-1} \sum_{j} S_{k j}^{\prime} \lambda_{j}^{\prime}=\mu_{r}^{(k i)}
$$

when $\sigma_{k} \neq 0$, while in the case with $\sigma_{k}=0$ we have

$$
\sum_{j, r^{\prime}}\left(M^{-1} \mu_{r}^{(k i)}+N^{-1} \mu_{r^{\prime}}^{(k j)}\right) \lambda_{j}^{\prime}=\mu_{r}^{(k i)}+N^{-1} \sum_{j} S_{k j}^{\prime} \lambda_{j}^{\prime}=\mu_{r}^{k i} .
$$

The counting of probabilities for the compositions $\left(f_{k i, r} \vee f_{k j, r^{\prime}}^{\prime}\right) \circ \mathcal{F}_{i j}^{\prime}=f_{k j, r^{\prime}}^{\prime}$ is analogous. Thus, we find that there is a unique choice of $\tilde{F}=F \vee F^{\prime}$ with the property that $\tilde{F} \circ \mathcal{F}=F$ and $\tilde{F} \circ \mathcal{F}^{\prime}=F^{\prime}$. Thus, we obtain that $\left(\Phi \amalg_{\lambda} \Phi^{\prime}\right) \circ \Psi=\Phi$ and $\left(\Phi \amalg_{\lambda} \Phi^{\prime}\right) \circ \Psi^{\prime}=\Phi^{\prime}$.

2.4. Probabilistic categories as wreath products. The same procedure we used to pass from the category of finite pointed sets $\mathcal{S}_{*}$ to its probabilistic counterpart $\mathcal{P} \mathcal{S}_{*}$ can be generalized to a procedure that associates to a category $\mathcal{C}$ with a zero object 0 and a categorical sum (coproduct) $\oplus$ a new category $\mathcal{P C}$, which is the probabilistic version of $\mathcal{C}$.

Definition 2.18. $\mathcal{P C}$ is the category whose objects are formal finite convex combinations

$$
\Lambda C=\sum_{i} \lambda_{i} C_{i}
$$

with $\Lambda=\left(\lambda_{i}\right)$ with $\sum_{i} \lambda_{i}=1$ and $C_{i} \in \operatorname{Obj}(\mathcal{C})$ and with morphisms $\Phi: \Lambda C \rightarrow \Lambda^{\prime} C^{\prime}$ given by pairs $\Phi=(S, F)$ with $S$ a stochastic matrix with $S \Lambda=\Lambda^{\prime}$ and $F=\left\{F_{a b, r}\right\}$ 
a finite collection of morphisms $F_{a b, r}: C_{b} \rightarrow C_{a}^{\prime}$ with assigned probabilities $\mu_{r}^{a b}$ with $\sum_{r} \mu_{r}^{a b}=S_{a b}$

As before, we interpret the collection $F$ as a mapping of $C_{a}$ to $C_{b}^{\prime}$ obtained by choosing one of the morphism in the collection $\left\{F_{a b, r}\right\}$ so that the probability of choosing $F_{a b, r}$ is $\mu_{r}^{a b}$.

Remark 2.19. The same argument used in Lemma 2.12 and Theorem 2.17 shows that if $\mathcal{C}$ has a zero object and a categorical sum, then $\mathcal{P C}$ also has a zero object, given by the zero object of $\mathcal{C}$ with $\Lambda=1$ and a categorical sum given by $\Lambda C \amalg \Lambda^{\prime} C^{\prime}=$ $\sum_{i, j} \lambda_{i} \lambda_{j}^{\prime} C_{i} \amalg_{\mathcal{C}} C_{j}^{\prime}$, which satisfies the the universal property, with $\Phi \amalg \Phi^{\prime}: \Lambda C \amalg \Lambda^{\prime} C^{\prime} \rightarrow$ $\Sigma C^{\prime \prime}$ given by $\Phi \amalg \Phi^{\prime}=\left(S \amalg S^{\prime}, F \amalg F^{\prime}\right)$, where $\left(S \amalg S^{\prime}\right)_{u,\left(a, a^{\prime}\right)}=\sigma_{u}^{-1} S_{u a} S_{u a^{\prime}}^{\prime}$ for $\sigma_{u} \neq 0$ and $\left(S \amalg S^{\prime}\right)_{u,\left(a, a^{\prime}\right)}=S_{u a}+S_{u, a^{\prime}}^{\prime}$ if $\sigma_{u}=0$, and with $F \amalg F^{\prime}=\left\{F_{u a, r} \amalg_{\mathcal{C}} F_{u a^{\prime}, r^{\prime}}\right\}$ with probabilities $\sigma_{u}^{-1} \mu_{r}^{u a} \mu_{r^{\prime}}^{u a^{\prime}}$ for $\sigma_{u} \neq 0$ and $M^{-1} \mu_{r}^{u a}+N^{-1} \mu_{r^{\prime}}^{u a^{\prime}}$ for $\sigma_{u}=0$.

Remark 2.20. The construction of $\mathcal{P C}$ from $\mathcal{C}$ can be seen as a wreath product $\mathcal{F P}$ \& $\mathcal{C}$ of the category $\mathcal{C}$ with the category $\mathcal{F P}$ of finite probabilities.

\section{INFORMATION LOSS}

We recall here some results about information loss from [2] that will be useful in the following sections.

3.1. Information loss. In classical information, the Shannon entropy of a measure $P=\left(P_{i}\right)$ on a finite set of cardinality $n$ is defined as

$$
S(P)=-\sum_{i=1}^{n} P_{i} \log P_{i} .
$$

It is well known that the function (3.1) can be characterized uniquely (up to an overall multiplicative constant $C>0$ ) by a set of simple axioms, the Khinchin axioms [20] expressing the properties of continuity, maximality at the equidistribution, additivity over subsystems $S(A \cup B)=S(A)+S(B \mid A)$, and expansibility (compatibility with respect to changing the dimension $n$ ). These axioms were also formulated in a more concise way in [9].

The Kullback-Leibler divergence, or relative entropy of two probability distributions $P, Q$ on the same finite set $\Sigma$ with $\# \Sigma=n$ is given by

$$
K L(P \| Q)=-\sum_{i} P_{i} \log \frac{Q_{i}}{P_{i}}
$$

It is not a metric (it is not symmetric, it can take infinite value, and does not satisfy a triangle inequality) but it defines a metric (the Fisher-Rao information metric) when considering the leading term in the expansion $K L\left(P+h \| P+h^{\prime}\right)$. In the case of probability distributions $P, Q$ on different sets related through a map $f: \Sigma \rightarrow \Sigma^{\prime}$, 
it is possible to compare them in a similar way via a particular case of conditional entropy $H(P \mid Q)$ (see $\S 3$ of [2]).

More precisely, consider then the category whose objects are finite sets $\Sigma$ with probability measures $P$ with morphisms $f:(\Sigma, P) \rightarrow\left(\Sigma^{\prime}, Q\right)$ given by measure preserving maps, satisfying

$$
Q_{j}=\sum_{i \in f^{-1}(j)} P_{i}
$$

Then the information loss of a morphism $f:(\Sigma, P) \rightarrow\left(\Sigma^{\prime}, Q\right)$ is defined as the conditional entropy

$$
\mathcal{I}(f)=\sum_{s \in \Sigma} P_{s} \log \frac{Q_{f(s)}}{P_{s}}=S(P)-S(Q) .
$$

The last equality holds as a consequence of the measure preserving assumption (3.3). The setting can be generalized by considering finite measures not necessarily normalized to probability measures, see [2], 3. The expression in (3.4) can be viewed as a Kullback-Leibler divergence between $P$ and a non-normalized pullback measure of $Q$ along $f$.

It was proved in [2] that the information loss function $\mathcal{I}(f)$ of $(3.4)$ satisfies an axiomatic characterization (up to a constant multiplicative factor), which follows from the Khinchin axioms of the Shannon entropy (as reformulated in [9]). The characterizing axioms in this setting are

- Additivity under composition of morphisms: $\mathcal{I}(f \circ g)=\mathcal{I}(f)+\mathcal{I}(g)$;

- Additivity under direct sums: $\mathcal{I}(f \oplus g)=\mathcal{I}(f)+\mathcal{I}(g)$;

- Homogeneity under scaling: $\mathcal{I}(\lambda f)=\lambda \mathcal{I}(f)$, for $\lambda \in \mathbb{R}_{+}^{*}$.

The last two properties are replaced by the single additivity over convex combinations

$$
\mathcal{I}(\lambda f \oplus(1-\lambda) g)=\lambda \mathcal{I}(f)+(1-\lambda) \mathcal{I}(g),
$$

for $\lambda \in[0,1]$, if the normalization of measures is preserved, see [2]. Additivity under composition plays the role of a functoriality property in the framework of [2].

3.2. Information loss and the category of stochastic maps. The argument of [2] on the unique characterization of the information loss functional can be easily adapted to the category $\mathcal{F} \mathcal{P}$ of finite probability measures introduced above.

The sets of morphisms $\operatorname{Mor}_{\mathcal{F P}}((X, P),(Y, Q))$ are convex sets, hence in particular they are topological spaces, so we can consider continuous functions on these sets.

Definition 3.1. An information loss functional on $\mathcal{F P}$ is a continuous real valued map on the set of morphisms $\mathcal{H}:$ Mor $_{\mathcal{F P}} \rightarrow \mathbb{R}$ with the properties

(1) the function $\mathcal{H}(S)=0$ on isomorphisms;

(2) for all $S \in \operatorname{Mor}_{\mathcal{F P}}((X, P),(Y, Q))$ and all $S^{\prime} \in \operatorname{Mor}_{\mathcal{F P}}\left((Y, Q),\left(Z, Q^{\prime}\right)\right)$

$$
\mathcal{H}\left(S^{\prime} \circ S\right)=\mathcal{H}\left(S^{\prime}\right)+\mathcal{H}(S)
$$


(3) for all $S \in \operatorname{Mor}_{\mathcal{F P}}((X, P),(Y, Q))$ and $S^{\prime} \in \operatorname{Mor}_{\mathcal{F P}}\left(\left(X^{\prime}, P^{\prime}\right),(Y, Q)\right)$ and $\lambda S \oplus$ $(1-\lambda) S^{\prime} \in \operatorname{Mor}_{\mathcal{F P}}\left(\left(X \sqcup X^{\prime}, \lambda P \oplus(1-\lambda) P^{\prime}\right),(Y, Q)\right)$

$$
\mathcal{H}\left(\lambda S \oplus(1-\lambda) S^{\prime}\right)=\lambda \mathcal{H}(S)+(1-\lambda) \mathcal{H}\left(S^{\prime}\right)+\mathcal{H}\left(\hat{1}_{(\lambda, 1-\lambda)}\right),
$$

for $\hat{1}_{(\lambda, 1-\lambda)}$ the unique morphism from $(\{x, y\},(\lambda, 1-\lambda))$ to the zero object.

With respect to the last property listed above, note that the operation of taking a disjoint union $X \sqcup X^{\prime}$ with the weighted sum of probabilities $\lambda P \oplus(1-\lambda) P^{\prime}$ is not the coproduct in the category. We discuss the behavior with respect to the coproduct in Corollary 3.5 below.

The following is essentially the same argument given in [2].

Lemma 3.2. For $S \in \operatorname{Mor}_{\mathcal{F}}((X, P),(Y, Q))$, setting $\mathcal{H}(S)=H(Q)-H(P)$, with $H(P)=-\sum_{x \in X} P_{x} \log P_{x}$ the Shannon entropy satisfies all the properties of Definition 3.1 .

Proof. The first three properties are clearly satisfied, since $\mathcal{H}(S)$ depends only on the source and target probabilities $P, Q$, through the difference of values of $H$ and the Shannon entropy of finite probability distributions is invariant under isomorphisms. The last property is satisfied because of the additivity over subsystems of the Shannon entropy, namely the property that, for all probabilities $P=\left(P_{1}, \ldots, P_{n}\right)$ and $Q_{i}$

$$
H\left(P_{1} Q_{1} \oplus \cdots \oplus P_{n} Q_{n}\right)=H(P)+\sum_{i=1}^{n} P_{i} H\left(Q_{i}\right) .
$$

In particular, for $P=(\lambda, 1-\lambda)$, we obtain

$$
H\left(\lambda P \oplus(1-\lambda) P^{\prime}\right)=H(\lambda, 1-\lambda)+\lambda H(P)+(1-\lambda) H\left(P^{\prime}\right) .
$$

This implies that, for $S \in \operatorname{Mor}_{\mathcal{F P}}((X, P),(Y, Q))$ and $S^{\prime} \in \operatorname{Mor}_{\mathcal{F P}}\left(\left(X^{\prime}, P^{\prime}\right),(Y, Q)\right)$

$$
H\left(\lambda S \oplus(1-\lambda) S^{\prime}\right)=H(Q)-\lambda H(P)-(1-\lambda) H\left(P^{\prime}\right)-H(\lambda, 1-\lambda),
$$

where $-H(\lambda, 1-\lambda)$ is identified with

$$
\mathcal{H}\left(\hat{1}_{(\lambda, 1-\lambda)}\right)=H(1)-H(\lambda, 1-\lambda),
$$

where $H(1)=0$.

Remark 3.3. Similarly, for $S \in \operatorname{Mor}_{\mathcal{F P}}((X, P),(Y, Q))$ and $S^{\prime} \in \operatorname{Mor}_{\mathcal{F P}}\left(\left(X, P^{\prime}\right),(Y, Q)\right)$ with $\lambda S+(1-\lambda) S^{\prime} \in \operatorname{Mor}_{\mathcal{F P}}\left(\left(X, \lambda P+(1-\lambda) P^{\prime}\right),(Y, Q)\right)$

$$
\mathcal{H}\left(\lambda S+(1-\lambda) S^{\prime}\right)=\lambda \mathcal{H}(S)+(1-\lambda) \mathcal{H}\left(S^{\prime}\right)+\mathcal{H}\left(\hat{1}_{(\lambda, 1-\lambda)}\right),
$$

and for $S \in \operatorname{Mor}_{\mathcal{F P}}((X, P),(Y, Q))$ and $S^{\prime} \in \operatorname{Mor}_{\mathcal{F P}}\left((X, P),\left(Y, Q^{\prime}\right)\right)$ and $\lambda S+(1-$ $\lambda) S^{\prime} \in \operatorname{Mor}_{\mathcal{F P}}\left((X, P),\left(Y, \lambda Q+(1-\lambda) Q^{\prime}\right)\right)$

$$
\mathcal{H}\left(\lambda S+(1-\lambda) S^{\prime}\right)=\lambda \mathcal{H}(S)+(1-\lambda) \mathcal{H}\left(S^{\prime}\right)-\mathcal{H}\left(\hat{1}_{(\lambda, 1-\lambda)}\right),
$$

while for $S \in \operatorname{Mor}_{\mathcal{F P}}((X, P),(Y, Q))$ and $S^{\prime} \in \operatorname{Mor}_{\mathcal{F P}}\left(\left(X, P^{\prime}\right),\left(Y, Q^{\prime}\right)\right)$ and $\lambda S+(1-$ $\lambda) S^{\prime} \in \operatorname{Mor}_{\mathcal{F P}}\left(\left(X, \lambda P+(1-\lambda) P^{\prime}\right),\left(Y, \lambda Q+(1-\lambda) Q^{\prime}\right)\right)$ we just have

$$
\mathcal{H}\left(\lambda S+(1-\lambda) S^{\prime}\right)=\lambda \mathcal{H}(S)+(1-\lambda) \mathcal{H}\left(S^{\prime}\right) .
$$


Proposition 3.4. The properties (3.6) and (3.7) determine uniquely the information loss functional, up to an overall non-zero multiplicative constant $C$. For a morphism $S \in \operatorname{Mor}_{\mathcal{F P}}((X, P),(Y, Q))$, the information loss is given by

$$
\mathcal{H}(S)=C \cdot(H(Q)-H(P)),
$$

where $H$ is the Shannon entropy

$$
H(P)=-\sum_{x \in X} P_{x} \log P_{x}
$$

Proof. The argument is essentially the same as in 2]. First observe that the composition of any morphism $S \in \operatorname{Mor}_{\mathcal{F P}}((X, P),(Y, Q))$ with the unique morphism $\hat{1}_{(Y, Q)}:(Y, Q) \rightarrow(\{x\}, 1)$ is

$$
\hat{1}_{(X, P)}=\hat{1}_{(Y, Q)} \circ S:(X, P) \rightarrow(\{x\}, 1) .
$$

Thus, property (3.6) gives

$$
\mathcal{H}(S)=\mathcal{H}\left(\hat{1}_{(X, P)}\right)-\mathcal{H}\left(\hat{1}_{(Y, Q)}\right) .
$$

It then suffices to show that

$$
\tilde{H}(P):=-\mathcal{H}\left(\hat{1}_{(X, P)}\right)=C \cdot H(P)
$$

is the Shannon entropy, up to a multiplicative constant. To this purpose it is sufficient to check that $\mathcal{H}\left(\hat{1}_{(X, P)}\right)$ satisfies the Khinchin axioms as formulated in [9]. The vanishing of $\mathcal{H}(S)$ on isomorphisms $S$ implies that $\mathcal{H}\left(\hat{1}_{(X, P)}\right)$ is invariant under isomorphisms of $(X, P)$. Invariance under isomorphisms also implies that $\mathcal{H}\left(\hat{1}_{(\{x\}, 1)}\right)=\tilde{H}(1)=0$. Continuity follows from the continuity of $\mathcal{H}(S)$. Thus, we only need to show that $\tilde{H}(P)$ satisfies the "additivity over subsystems" property of the Shannon entropy,

$$
\tilde{H}\left(P_{1} Q_{1}, \ldots, P_{n} Q_{n}\right)=\tilde{H}(P)+\sum_{i=1}^{n} P_{i} \tilde{H}\left(Q_{i}\right) .
$$

We proceed inductively. We have

$$
\tilde{H}\left(\lambda Q,(1-\lambda) Q^{\prime}\right)=\tilde{H}(\lambda, 1-\lambda)+\lambda \tilde{H}(Q)+(1-\lambda) \tilde{H}\left(Q^{\prime}\right)
$$

since

$$
-\tilde{H}\left(\lambda Q,(1-\lambda) Q^{\prime}\right)=\tilde{H}(1)-\tilde{H}\left(\lambda Q,(1-\lambda) Q^{\prime}\right)=-\mathcal{H}\left(\lambda \hat{1}_{(Y, Q)} \oplus(1-\lambda) \hat{1}_{\left(Y^{\prime}, Q^{\prime}\right)}\right),
$$

with $\hat{1}:(Y, Q) \rightarrow(\{x\}, 1)$ and $\hat{1}_{\left(Y^{\prime}, Q^{\prime}\right)}:\left(Y^{\prime}, Q^{\prime}\right) \rightarrow(\{x\}, 1)$, and with $\tilde{H}(1)=0$. Then we write

$$
\begin{gathered}
\tilde{H}\left(P_{0} Q_{0}, P_{1} Q_{1}, \ldots, P_{n} Q_{n}\right)=\tilde{H}\left(\lambda Q,(1-\lambda) P_{1}^{\prime} Q_{1}, \ldots,(1-\lambda) P_{n}^{\prime} Q_{n}\right) \\
=\tilde{H}(\lambda, 1-\lambda)+\lambda \tilde{H}(Q)+(1-\lambda) \tilde{H}\left(P_{1}^{\prime} Q_{1}, \ldots, P_{n}^{\prime} Q_{n}\right) \\
=\tilde{H}(\lambda, 1-\lambda)+\lambda \tilde{H}(Q)+(1-\lambda)\left(\tilde{H}\left(P^{\prime}\right)+\sum_{i} P_{i}^{\prime} \tilde{H}\left(Q_{i}\right)\right) .
\end{gathered}
$$

We identify

$$
\tilde{H}\left(P_{0}, \ldots, P_{n}\right)=\tilde{H}\left(\lambda,(1-\lambda) P^{\prime}\right)=-\mathcal{H}\left(\lambda \hat{1}_{(\{x\}, 1)} \oplus(1-\lambda) \hat{1}_{\left(X^{\prime}, P^{\prime}\right)}\right)
$$


hence we obtain

$$
\tilde{H}(\lambda, 1-\lambda)+\lambda \tilde{H}(1)+(1-\lambda) \tilde{H}\left(P^{\prime}\right)
$$

so that

$$
\begin{gathered}
\left.\tilde{H}\left(P_{0} Q_{0}, P_{1} Q_{1}, \ldots, P_{n} Q_{n}\right)=\tilde{H}\left(\lambda,(1-\lambda) P^{\prime}\right)+\lambda \tilde{H}(Q)+(1-\lambda) \sum_{i=1}^{n} P_{i}^{\prime} \tilde{H}\left(Q_{i}\right)\right) \\
=\tilde{H}\left(P_{0}, \ldots, P_{n}\right)+\sum_{i=0}^{n} P_{i} \tilde{H}\left(Q_{i}\right) .
\end{gathered}
$$

Corollary 3.5. The information loss functional evaluated on the coproduct $S \amalg S^{\prime}$ of morphisms $S:(X, P) \rightarrow(Y, Q)$ and $S^{\prime}:\left(X^{\prime}, P^{\prime}\right) \rightarrow(Y, Q)$ satisfies

$$
\mathcal{H}\left(S \amalg S^{\prime}\right)=\mathcal{H}(S)+\mathcal{H}\left(S^{\prime}\right)-\mathcal{H}(\hat{Q}),
$$

with $\hat{Q}:(\{x\}, 1) \rightarrow(Y, Q)$ the unique morphism from the zero object.

Proof. We have

$$
\mathcal{H}\left(S \amalg S^{\prime}\right)=H(Q)-H(P)-H\left(P^{\prime}\right)=\mathcal{H}(S)+\mathcal{H}\left(S^{\prime}\right)-H(Q),
$$

where we identify $H(Q)=H(Q)-H(1)=\mathcal{H}(\hat{Q})$.

\section{Gamma SPACES AND Finite PROBABIlities}

We have seen in the previous section that the category $\mathcal{P} \mathcal{S}_{*}$ of probabilistic pointed sets and the category $\mathcal{F} \mathcal{P}$ of finite probability spaces have a zero object and a categorical sum. Thus, one can apply to both of these categories the Segal construction of $\Gamma$-spaces.

4.1. Gamma spaces. We recall the main idea of the Segal construction of $\Gamma$-spaces, [30]. A $\Gamma$-space is a functor $F: \Gamma^{0} \rightarrow \Delta_{*}$ from the category of pointed finite sets to the category of pointed simplicial sets.

Given a category $\mathcal{C}$ with zero object and categorical sum, one can construct an associated $\Gamma$-space $F_{\mathcal{C}}: \Gamma^{0} \rightarrow \Delta_{*}$ in the following way. For a given pointed set $X \in \Gamma^{0}$ one considers the category $\Sigma_{\mathcal{C}}(X)$ of summing functors $\Phi_{X}: P(X) \rightarrow \mathcal{C}$, where $P(X)$ is the category with objects the pointed subsets of $X$ and morphisms the inclusions and the functors satisfy the summing properties

(1) $\Phi_{X}(\star)=0$, the base point of $X$ maps to the zero orbject of $\mathcal{C}$

(2) $\Phi_{X}\left(A \cup A^{\prime}\right)=\Phi_{X}(A) \amalg \Phi_{X}\left(A^{\prime}\right)$ for any two points sets with $A \cap A^{\prime}=\{\star\}$ and with $\amalg$ the categorical sum of $\mathcal{C}$. 
The morphisms of $\Sigma_{\mathcal{C}}(X)$ are natural transformations that are isomorphisms. The simplicial set $F_{\mathcal{C}}(X)=\mathcal{N} \Sigma_{\mathcal{C}}(X)$ is the nerve of the category of summing functors.

Given a functor $F: \mathcal{C}^{o p} \times \mathcal{C} \rightarrow \mathcal{D}$, the coend $\int^{C \in \mathcal{C}} F(C, C)$ is the initial cowedge, where a cowedge to an object $X$ in $\mathcal{C}$ is a family of morphisms $h_{A}: A \rightarrow X$, for each $A \in \mathcal{C}$, such that, for all morphisms $f: A \rightarrow B$ in $\mathcal{C}$ the following diagrams commute

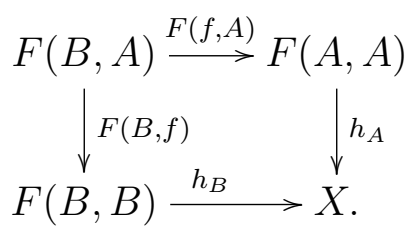

Given a $\Gamma$-space $F: \Gamma^{0} \rightarrow \Delta_{*}$, it is possible to extend it to an endofunctor $F$ : $\Delta_{*} \rightarrow \Delta_{*}$. Let $X_{n}$ denote the finite pointed set in $\Gamma^{0}$ with $\# X_{n}=n+1$. The endofunctor of $\Delta_{*}$ is obtained [4] (see also [29]) as the functor (still denoted by $F$ ) that maps a pointed simpliciat set $K \in \Delta_{*}$ to the coend

$$
F: K \mapsto \int^{X_{n} \in \Gamma^{0}} K_{n} \wedge F\left(X_{n}\right),
$$

with natural assembly maps $K \wedge F\left(K^{\prime}\right) \rightarrow F\left(K \wedge K^{\prime}\right)$. Here the smash product $K_{n} \wedge F\left(X_{n}\right)$ attaches a copy of the simplicial set $F\left(X_{n}\right)$ to each element in the set $K_{n}$, which is the list of $n$-simplexes of $K$. Taking the coend then ensures that these are glued together correctly according to the prescription of the face and degeneracy maps of the simplicial set $K$.

The spectrum associated to the $\Gamma$-space is then obtained by considering the simplicial sets given by the spheres $S^{n}=S^{1} \wedge \cdots \wedge S^{1}$, with the simplicial structure induced by the simplicial circle $S^{1}=\Delta_{1} / \partial \Delta_{1}$. The maps $K^{\prime} \wedge F(K) \rightarrow F\left(K^{\prime} \wedge K\right)$ give rise to the structure maps $S^{1} \wedge F\left(S^{n}\right) \rightarrow F\left(S^{n+1}\right)$ of the spectrum defined by the sequence of pointed simplicial sets $X_{n}=F\left(S^{n}\right)$.

4.2. Cubical sets. It is possible to reformulate homotopy constructions in terms of cubical sets rather than simplicial sets. Heuristically, while simplicial sets are modeled on the combinatorics of finite sets, cubical sets are modeled on power sets. We will see why this shift of viewpoint is relevant to the setting of probabilistic pointed sets.

We recall the basic definition of cubical sets, [5], [18], [19]. Let $\mathcal{I}$ be the unit interval, considered as the combinatorial structure consisting of two vertices and an edge connecting them. We also write $|\mathcal{I}|=[0,1]$ for the geometric unit interval given by its realization. Similarly, we write $\mathcal{I}^{n}$ for the $n$-cube, and $\left|\mathcal{I}^{n}\right|=[0,1]^{n}$ for its geometric realization, for any $n \geq 0$, with $\mathcal{I}^{0}$ a single point. The face maps $\delta_{i}^{a}: \mathcal{I}^{n} \rightarrow \mathcal{I}^{n+1}$, for $a \in\{0,1\}$ and $i=1, \ldots, n$ are given by

$$
\delta_{i}^{a}\left(t_{1}, \ldots, t_{n}\right)=\left(t_{1}, \ldots, t_{i-1}, a, t_{i}, \ldots, t_{n}\right)
$$

while the degeneracy maps $s_{i}: \mathcal{I}^{n} \rightarrow \mathcal{I}^{n-1}$ are given by

$$
s_{i}\left(t_{1}, \ldots, t_{n}\right)=\left(t_{1}, \ldots, t_{i-1}, t_{i+1}, \ldots, t_{n}\right) .
$$


These maps satisfy the cubical relations for $i<j$

$$
\delta_{j}^{b} \circ \delta_{i}^{a}=\delta_{i}^{a} \circ \delta_{j-1}^{b} \quad \text { and } \quad s_{i} \circ s_{j}=s_{j-1} \circ s_{i}
$$

as well as the relations

$$
\begin{array}{ll}
\delta_{i}^{a} \circ s_{j-1}=s_{j} \circ \delta_{i}^{a} & i<j \\
s_{j} \circ \delta_{i}^{a}=1 & i=j \\
\delta_{i-1}^{a} \circ s_{j}=s_{j} \circ \delta_{i}^{a} & i>j
\end{array}
$$

The cube category $\mathfrak{C}$ has objects $\mathcal{I}^{n}$ for $n \geq 0$ and morphisms generated by the face and degeneracy maps $\delta_{i}^{a}$ and $s_{i}$. A cubical set is a functor $C: \mathfrak{C}^{o p} \rightarrow \mathcal{S}$ to the category of sets. We write $C_{n}=C\left(\mathcal{I}^{n}\right)$.

One can enlarge the category $\mathfrak{C}$ to a category $\mathfrak{C}_{c}$ that has additional degeneracy maps $\gamma_{i}: \mathcal{I}^{n} \rightarrow \mathcal{I}^{n-1}$ called connections (see [5])

$$
\gamma_{i}\left(t_{1}, \ldots, t_{n}\right)=\left(t_{1}, \ldots, t_{i-1}, \max \left\{t_{i}, t_{i+1}\right\}, t_{i+2}, \ldots, t_{n}\right) .
$$

These satisfy the relations

$$
\begin{gathered}
\gamma_{i} \gamma_{j}=\gamma_{j} \gamma_{i+1}, i \leq j ; \quad s_{j} \gamma_{i}= \begin{cases}\gamma_{i} s_{j+1} & i<j \\
s_{i}^{2}=s_{i} s_{i+1} & i=j \\
\gamma_{i-1} s_{j} & i>j\end{cases} \\
\gamma_{j} \delta_{i}^{a}= \begin{cases}\delta_{i}^{a} \gamma_{j-1} & i<j \\
1 & i=j, j+1, a=0 \\
\delta_{j}^{a} s_{j} & i=j, j+1, a=1 \\
\delta_{i-1}^{a} \gamma_{j} & i>j+1 .\end{cases}
\end{gathered}
$$

The connection maps $\gamma_{i}$ are extra degeneracies. While the usual degeneracy maps $s_{i}$ identify opposite faces of a cube these additional degeneracies identify adjacent faces.

A cubical set with connection is a functor $C: \mathfrak{C}_{c}^{o p} \rightarrow \mathcal{S}$ to the category of sets.

The category of cubical sets has these functors as objects and natural transformations as morphisms, that is, a collection $\alpha=\left(\alpha_{n}\right)$ of morphisms $\alpha_{n}: C_{n} \rightarrow C_{n}^{\prime}$ satisfying the compatibilities $\alpha \circ \delta_{i}^{a}=\delta_{i}^{a} \circ \alpha$ and $\alpha \circ s_{i}=s_{i} \circ \alpha$ (and in the case with connection $\left.\alpha \circ \gamma_{i}=\gamma_{i} \circ \alpha\right)$.

It is convenient to work with cubical sets with connection, as this corrects the problem that the realization functor from cubical sets to topological spaces does not preserve finite products. Moreover, when working with cubical sets with connection, as shown in [1, there is a cubical classifying space and cubical nerve construction (see also [10]) which is homotopy equivalent to the usual simplicial one.

We refer to the cubical nerve of a category $\mathcal{C}$ with the notation $\mathcal{N}_{\mathfrak{C}} \mathcal{C}$. It is defined as the cubical set with

$$
\left(\mathcal{N}_{\mathfrak{C}} \mathcal{C}\right)_{n}=\operatorname{Fun}\left(\mathcal{I}^{n}, \mathcal{C}\right)
$$

where $\mathcal{I}^{n}$ is the $n$-cube seen as a category with objects the vertices and morphisms generated by the 1 -faces (edges), and $\operatorname{Fun}\left(\mathcal{I}^{n}, \mathcal{C}\right)$ is the set of functors from $\mathcal{I}^{n}$ to $\mathcal{C}$. 
4.3. Cubical Gamma spaces. We can consider the analog of $\Gamma$-spaces in the cubical setting.

Definition 4.1. Let $\Gamma=\mathcal{S}_{*}$ be the category of pointed sets. A pointed cubical set with connection is a functor $K: \mathfrak{C}_{c}^{o p} \rightarrow \mathcal{S}_{*}$. We denote by $K_{n}=K\left(\mathcal{I}^{n}\right)$ with $\star \in K_{n}$ the base point. We denote by $\square_{*}$ the category of pointed cubical sets with connection, with objects the functors $K: \mathfrak{C}_{c}^{o p} \rightarrow \mathcal{S}_{*}$ and morphisms the natural transformations. A cubical $\Gamma$-space is a functor $F: \Gamma^{0} \rightarrow \square_{*}$.

The Segal construction can be adapted to obtain cubical $\Gamma$-spaces from categories with zero object and a categorical sum. One proceeds in the same way, by constructing the category of summing functors $\Sigma_{\mathcal{C}}(X)$ for finite pointed sets $X \in \Gamma^{0}$, and then one takes the cubical nerve $\mathcal{N}_{\mathfrak{C}} \Sigma_{\mathcal{C}}(X)$. The resulting cubical $\Gamma$ space $F_{\mathcal{C}}^{\mathfrak{C}}: \Gamma^{0} \rightarrow \square_{*}$ assigns to a pointed finite set $X$ the pointed cubical set with connection $F_{\mathcal{C}}^{\mathfrak{C}}(X)=\mathcal{N}_{\mathfrak{C}} \Sigma_{\mathcal{C}}(X)$.

Since $\mathcal{N}_{\mathfrak{C}} \Sigma_{\mathcal{C}}(X)$ is homotopy equivalent to the simplicial nerve $\mathcal{N} \Sigma_{\mathcal{C}}(X)$ (see [1]) there is no loss of generality in adopting this cubical setting for $\Gamma$-spaces.

4.4. The summing functors of probabilistic pointed sets. We consider again the category $\mathcal{P} \mathcal{S}_{*}$ of probabilistic pointed set. For a choice of a finite pointed set $X \in \Gamma$, we construct the category of summing functors $\Sigma_{\mathcal{P} \mathcal{S}_{*}}(X)$.

Theorem 4.2. Objects in the category $\Sigma_{\mathcal{P} \mathcal{S}_{*}}(X)$ of summing functors can be identified with choices of a point $\Lambda=\left\{\lambda_{x}\right\}_{x \in X \backslash\{\star\}} \in\left|\mathcal{I}^{N}\right|$, with $\# X=N+1$, with the summing functor $\Phi_{\Lambda}(A)=\Lambda_{A} X_{A}$ a combination of $2^{N_{A}}$ pointed sets of cardinality $N_{A}=\# A-1$, with probability distribution

$$
\Lambda_{A}=\left\{\left(t_{1}, \ldots, t_{N_{A}}\right): t_{x} \in\left\{\lambda_{x},\left(1-\lambda_{x}\right)\right\}\right\},
$$

with morphisms given by permutations of the $2^{N_{A}}$ sequences in (4.8).

Proof. Given a finite pointed set $X \in \Gamma$ with base point $\star$, let $P(X)$ be the category with objects the pointed subsets of $X$ and morphisms given by inclusions. The objects of $\Sigma_{\mathcal{P} \mathcal{S}_{*}}(X)$ consist of functors $\Phi: P(X) \rightarrow \mathcal{P} \mathcal{S}_{*}$ satisfying $\Phi(\star)=(\{x\}, x)$, the zero object of $\mathcal{P} \mathcal{S}_{*}$ with $\Lambda=1$, and, for any sets $A, B \in P(X)$ with $A \cap B=\{\star\}$,

$$
\Phi(A \cup B)=\Phi(A) \amalg \Phi(B),
$$

the coproduct in $\mathcal{P S}_{*}$. In particular the condition (4.9) implies that, given a pointed subset $A \in P(X)$ the value $\Phi(A)$ is given by

$$
\Phi(A)=\amalg_{a \in A \backslash\{\star\}} \Phi(\{\star, a\}) .
$$

Given a morphism in $P(X)$, namely a pointed inclusion $j: A \hookrightarrow A^{\prime}$, we write $A^{\prime}=A \vee B$ with $B=\left(A^{\prime} \backslash A\right) \cup\{\star\}$ so that $\Phi\left(A^{\prime}\right)=\Phi(A) \amalg \Phi(B)$. Then the morphism $\Phi(j)$ in $\mathcal{P} \mathcal{S}_{*}$ is the map $\Psi=(\mathcal{I}, \mathcal{F})$ to the coproduct as in Theorem 2.17

$$
\Phi(j)=\Psi=(\mathcal{I}, \mathcal{F}): \Phi(A) \rightarrow \Phi(A) \amalg \Phi(B) .
$$

In terms of probabilistic pointed sets, when we consider the union $X_{a} \cup X_{b}$ of two sets of the form $X_{a}=\{\star, a\}$ and $X_{b}=\{\star, b\}$, this means that we select for both 
$X_{a}$ and $X_{b}$ the point $\star$ as base point and then we take the coproduct of pointed sets $\left(X_{a}, \star\right) \vee\left(X_{b}, \star\right)=\{\star, a, b\}$. We can think of $X_{a}$ and $X_{b}$ as probabilistic pointed sets of the form $\Lambda X_{a}=\lambda_{a}\left(X_{a}, \star\right)+\left(1-\lambda_{a}\right)\left(X_{a}, a\right)$ and $\Lambda X_{b}=\lambda_{b}\left(X_{b}, \star\right)+\left(1-\lambda_{b}\right)\left(X_{b}, b\right)$, where $\lambda_{a}$ and $\lambda_{b}$ in $[0,1]$ are the respective probabilities of selecting $\star$ as the basepoint, instead of $a$ or $b$. Thus, when we describe a pointed subset $A \subset X$ as the coproduct of pointed sets $A=\vee_{a \in A} X_{a}$ we are selecting for each $X_{a}$ the same basepoint $\star$, when we view them as probabilistic pointed sets. Thus, $A$ can be obtained from the probabilistic pointed sets $\left\{\Lambda X_{a}\right\}_{a \in A \backslash\{\star\}}$ with probability

$$
\lambda_{A}=\prod_{a \in A \backslash\{\star\}} \lambda_{a}
$$

If $\lambda_{x} \neq 0$ for all $x \in X$, these probabilities satisfy the multiplicative inclusionexclusion relation

$$
\lambda_{A \cup B}=\frac{\lambda_{A} \cdot \lambda_{B}}{\lambda_{A \cap B}}
$$

Given a pointed subset $A \in P(X)$ and an assignment of values $\left\{\lambda_{a}\right\}_{a \in A \backslash\{\star\}}$, with $\lambda_{a} \in(0,1)$, there is a uniquely determined probabilistic pointed set, which we denote by $\Lambda_{A} X_{A}$, such that the pointed set $A$ occurs in the combination $\Lambda_{A} X_{A}$ with probability $\prod_{a \in A \backslash\{\star\}} \lambda_{a}$. For example, for $A=\{\star, a, b\}$, the associated probabilistic pointed set is of the form

$$
\begin{gathered}
\Lambda_{A} X_{A}=\lambda_{a} \lambda_{b}(\{\star, a\} \vee\{\star b\}, \star \sim \star)+\lambda_{a}\left(1-\lambda_{b}\right)(\{\star, a\} \vee\{\star b\}, \star \sim b) \\
+\left(1-\lambda_{a}\right) \lambda_{b}(\{\star, a\} \vee\{\star b\}, a \sim \star)+\left(1-\lambda_{a}\right)\left(1-\lambda_{b}\right)(\{\star, a\} \vee\{\star b\}, a \sim b) .
\end{gathered}
$$

Similarly for $\# A=N_{A}+1$, the probabilistic pointed set $\Lambda_{A} X_{A}$ is a combination of $2^{N_{A}}$ terms with probability distribution $\Lambda_{A}=\left\{t_{1} \cdots t_{\# A}: t_{a} \in\left\{\lambda_{a},(1-\lambda)_{a}\right\}\right\}$.

This shows that in order to construct a summing functor $\Phi: P(X) \rightarrow \mathcal{P} \mathcal{S}_{*}$ it suffices to assign a choice of coefficients $\left\{\lambda_{x}\right\}_{x \in X \backslash\{\star\}} \in\left|\mathcal{I}^{N}\right|$ with $N=\# X-1$ and that, conversely, a summing functor determines a collection of $\lambda_{x}$ as the probabilities assigned to the pointed sets $\{\star, a\}$ in $\Phi(\{\star, a\})=\Lambda X_{a}$. Morphisms in the category $\Sigma_{\mathcal{P} \mathcal{S}_{*}}(X)$ are natural transformations that are isomorphisms on objects, $\eta_{A}: \Phi(A) \stackrel{\widetilde{\sim}}{\rightarrow} \Phi^{\prime}(A)$ compatible with inclusions $j: A \hookrightarrow A^{\prime}$, with $\Phi^{\prime}(j) \circ \eta_{A}=\eta_{A^{\prime}} \circ \Phi(j)$. These are morphisms $\eta_{A}: \Lambda_{A} X_{A} \rightarrow \Lambda_{A}^{\prime} X_{A}^{\prime}$ in $\mathcal{P} \mathcal{S}_{*}$, with $\eta_{A}=\left(S_{A}, F_{A}\right)$ with $S_{A} \in \operatorname{Mor}_{\mathcal{F P}}\left(\Lambda_{A}, \Lambda_{A}^{\prime}\right)$ an isomorphism and $F_{A}=\left\{\left(F_{A}\right)_{a b, r}\right\}$ a collection of pointed isomorphisms between the pointed sets in the combination $X_{A}$ and the pointed sets in the combination $X_{A}^{\prime}$, with probabilities $\sum_{r} \mu_{r}^{(a b)}=\left(S_{A}\right)_{a b}$. The only stochastic matrices with a stochastic inverse are permutation matrices, hence the probabilities $\Lambda_{A}$ and $\Lambda_{A}^{\prime}$ are related by a permutation so $\left(S_{A}\right)_{a b}$ are either 0 or 1 . Thus, the underlying pointed sets are identified by isomorphisms $F_{a, r}$ with probabilities $\sum_{r} \mu_{r}=1$. The probability $\Lambda_{A}$ consists of all sequences $t_{1} \cdots t_{N}$ with $t_{i} \in\left\{\lambda_{a_{i}},\left(1-\lambda_{a_{i}}\right)\right\}$, for $\# A=N+1$ and $\left\{\lambda_{x}\right\}_{x \in X \backslash\{\star\}}$ specifying the summing functor $\Phi$. Thus, a permutation relating $\Lambda_{A}$ and $\Lambda_{A^{\prime}}$, compatibly with morphisms $\Phi(j)$ and $\Phi^{\prime}(j)$ induced by the inclusions $j: A \hookrightarrow B$, is a permutation of the $2^{N}$ sequences $t_{1} \cdots t_{N}$. 
Proposition 4.3. The cubical nerve $\mathcal{N}_{\mathfrak{C}} \Sigma_{\mathcal{P S}}(X)$ has sets

$$
K_{n}=\left(\mathcal{N}_{\mathfrak{C}} \Sigma_{\mathcal{P S}}(X)\right)_{n}=\operatorname{Fun}\left(\mathcal{I}^{n}, \Sigma_{\mathcal{P S}}(X)\right)
$$

given by all the assignments of a pair $\left(\Lambda, \mathbb{P}_{n}\right)$ consisting of a point $\Lambda=\left\{\lambda_{x}\right\}_{x \in X \backslash\{\star\}} \in$ $\left|\mathcal{I}^{\# X-1}\right|$ and a pointed polytope with $2^{n}$ vertices $\mathbb{P}_{n}=\left\{\left(t_{1}, \ldots, t_{n}\right): t_{i} \in\left\{\lambda_{x_{i}}, 1-\right.\right.$ $\left.\left.\lambda_{x_{i}}\right\}\right\}$, and distinguished vertex the sequence $\left(\lambda_{x_{1}}, \ldots, \lambda_{x_{n}}\right)$.

Proof. The cubical nerve of the category $\Sigma_{\mathcal{P S}}(X)=\left\{\Sigma_{\mathcal{P} \mathcal{S}_{*}}(X)_{n}\right\}$ is constructed by considering, for all $n \geq 0$, the set of functors $\Theta: \mathcal{I}^{n} \rightarrow \Sigma_{\mathcal{P} \mathcal{S}_{*}}(X)$. The objects of $\mathcal{I}^{n}$ are the vertices of the cube $\mathcal{I}^{n}$, namely all the sequences $v=\left(s_{1}, \ldots, s_{n}\right)$ with $s_{i} \in\{0,1\}$. To each vertex $v$ of $\mathcal{I}^{n}$, a functor $\Theta: \mathcal{I}^{n} \rightarrow \Sigma_{\mathcal{P S}}(X)$ associates an object of $\Sigma_{\mathcal{P S}}(X)$, that is, a summing functor $\Phi_{v}: P(X) \rightarrow \mathcal{P} \mathcal{S}_{*}$, which is specified, as above, by the choice of a point $\left\{\lambda_{x}\right\}_{x \in X \backslash\{\star\}}$ in the realization cube $\left|\mathcal{I}^{\# X-1}\right|$. The morphisms in $\mathcal{I}^{n}$ are generated by the edges $e$ of the cube and the image of any of these morphisms under $\Theta$ is a natural transformation of functors $\Phi_{v}$ and $\Phi_{v^{\prime}}$ associated to adjacent vertices $\left\{v, v^{\prime}\right\}=\partial e$ of $\mathcal{I}^{n}$. These natural transformations are permutations, corresponding to the exchange of the sequences $v, v^{\prime} \in\{0,1\}^{n}$, relating the $2^{n}$ sequences of $\Lambda_{A} X_{A}=\Phi_{v}(A)$ and $\Lambda_{A}^{\prime} X_{A}^{\prime}=\Phi_{v^{\prime}}(A)$. Thus, we can identify the datum of a functor $\Theta: \mathcal{I}^{n} \rightarrow \Sigma_{\mathcal{P} \mathcal{S}_{*}}(X)$ with the assignment of a point $\left\{\lambda_{x}^{v}\right\}_{x \in X \backslash\{\star\}} \in\left|\mathcal{I}^{\# X-1}\right|$ at each vertex $v \in \mathcal{I}^{n}$ together with a product probability space

$$
\mathbb{P}(v)=\prod_{i=1}^{n}\left(\lambda_{x_{i}}^{v}, 1-\lambda_{x_{i}}^{v}\right)=\left(\{0,1\}^{n},\left(t_{1}, \ldots, t_{n}\right)_{t_{i} \in\left\{\lambda_{x_{i}}^{v}, 1-\lambda_{x_{i}}^{v}\right\}}\right) .
$$

The set $\mathbb{P}(v)$ has a distinguished point given by the sequence $\left(\lambda_{x_{1}}, \ldots, \lambda_{x_{n}}\right)$, by 4.11$)$. The sequences $\Lambda^{v}$ and $\Lambda^{v^{\prime}}$ associated to adjacent vertices are related by the permutation that exchanges $v=\left(s_{1}, \ldots, s_{n}\right)$ and $v^{\prime}=\left(s_{1}^{\prime}, \ldots, s_{n}^{\prime}\right)$ in $\{0,1\}^{n}$, so that the sequences $\left(\lambda_{x_{1}}^{v}, \ldots, \lambda_{x_{n}}^{v}\right)$ and $\left(\lambda_{x_{1}}^{v^{\prime}}, \ldots, \lambda_{x_{n}}^{v^{\prime}}\right)$ are related by the same permutation. Adjacent vertices $v, v^{\prime}$ are sequences in $\{0,1\}^{n}$ that differ at a single digit. Thus, the condition implies that the corresponding sequences $\Lambda^{v}$ and $\Lambda^{v^{\prime}}$ also differ at a single $x_{i}$ where $\lambda_{x_{i}}$ is exchanged with $\left(1-\lambda_{x_{i}}\right)$. We can then identify the pairs $\left(\Lambda^{v}, \mathbb{P}(v)\right)$ consisting of a point $\Lambda^{v}=\left\{\lambda_{x}^{v}\right\}_{x \in X} \in\left|\mathcal{I}^{\# X}\right|$ and a pointed polytope with $2^{n}$ vertices $\mathbb{P}(v)=\left\{\left(t_{1}, \ldots, t_{n}\right): t_{i} \in\left\{\lambda_{x_{i}}^{v}, 1-\lambda_{x_{i}}^{v}\right\}\right.$, that satisfy these permutation conditions for all adjacent vertices with a single choice of $\Lambda=\left\{\lambda_{x}\right\}_{x \in X \backslash\{\star\}} \in\left|\mathcal{I}^{\# X-1}\right|$ (independent of $n)$ and a single pointed polytope $\mathbb{P}_{n}=\left\{\left(t_{1}, \ldots, t_{n}\right): t_{i} \in\left\{\lambda_{x_{i}}, 1-\lambda_{x_{i}}\right\}\right\}$ with base point $\left(\lambda_{x_{1}}, \ldots, \lambda_{x_{n}}\right)$.

The geometric realization $|K|$ of $K=\left\{K_{n}\right\}$ is obtained as

$$
|K|=\bigcup_{n=1}^{\infty} K_{n} \times\left|\mathcal{I}^{n}\right| / \sim
$$

with the relation identifying faces $\left(x, \delta_{i}^{a}(t)\right) \sim\left(\delta_{i}^{a}(x), t\right)$, degeneracies $\left(x, s_{i}(t)\right) \sim$ $\left(s_{i}(x), t\right)$, and connections $\left(x, \gamma_{i}(t)\right)=\left(\gamma_{i}(x), t\right)$. 
Proposition 4.4. The geometric realization $\left|\mathcal{N}_{\mathfrak{C}} \Sigma_{\mathcal{P S}}(X)\right|$ is given by

$$
\bigcup_{Z \in\left|\mathcal{I}^{N}\right|}\left|\mathcal{I}_{Z}^{N}\right|
$$

where $\# X=N+1$ and $\left|\mathcal{I}_{Z}^{N}\right|$ the geometric $N$-cube with vertices $\left\{\left(t_{1}, \ldots, t_{N}\right): t_{i} \in\right.$ $\left.\left\{z_{i}, 1-z_{i}\right\}\right\}$, for $Z=\left\{z_{i}\right\}_{i=1}^{N} \in\left|\mathcal{I}^{N}\right|=[0,1]^{N}$.

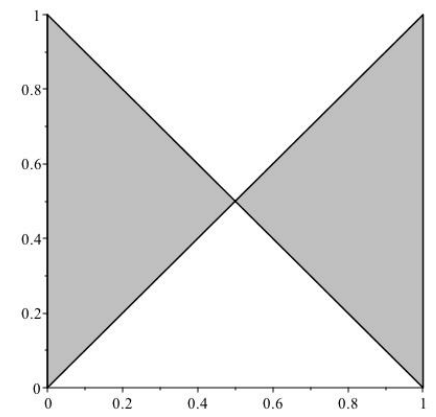

Figure 1. The realization $\left|\mathcal{N}_{\mathfrak{C}} \Sigma_{\mathcal{P} \mathcal{S}_{*}}(X)\right|$ for $X=\{\star, x\}$.

4.5. Smash product. The category $\mathcal{S}_{*}$ of pointed finite sets has a smash product operation given by $(X, x) \wedge(Y, y)=(X \times Y /(X \times\{y\} \cup\{x\} \times Y)$, z) with $z$ the base point obtained from quotienting the subspace $X \times\{y\} \cup\{x\} \times Y$.

This extends to a smash product $K \wedge K^{\prime}$ of pointed cubical sets $K, K^{\prime}: \mathfrak{C}_{c}^{o p} \rightarrow \mathcal{S}_{*}$, as in the case of pointed simplicial sets.

The smash product can be extended to the category $\mathcal{P} \mathcal{S}_{*}$ by taking

$$
\Lambda X \wedge \Lambda^{\prime} X^{\prime}=\sum_{i j} \lambda_{i} \lambda_{j}^{\prime}\left(X_{i} \wedge Y_{j}, z_{i j}\right)
$$

for $\Lambda X=\sum_{i} \lambda_{i}\left(X, x_{i}\right)$ and $\Lambda^{\prime} X^{\prime}=\sum_{j} \lambda_{j}^{\prime}\left(X_{j}^{\prime}, x_{j}^{\prime}\right)$.

Note, however, that as observed in $\$ 2.2 .4$ the product of statistically independent probability spaces does not satisfy the universal property of a categorical product.

\section{Stochastic Gamma spaces}

In the previous section we constructed a category $\mathcal{P} \mathcal{S}_{*}$ of probabilistic pointed sets and a related category $\mathcal{F} \mathcal{P}$ of finite probability spaces, which have with zero objects and a categorical sum and we have applied the Segal construction of Gamma spaces [30]. The resulting $\Gamma$ space $F_{\mathcal{P} \mathcal{S}_{*}}$ can be seen as a generalization of the $\Gamma$-space $F_{\mathcal{S}_{*}}$ associated to the sphere spectrum, with $\mathcal{S}_{*}$ the category of pointed finite sets. 
Here we change the viewpoint, and we modify the Segal construction in the same manner in which we have modified the category $\Gamma^{0}$ of pointed finite sets to its probabilistic version $\mathcal{P} \mathcal{S}_{*}$. Namely we make both the notion of Gamma spaces and the associated construction of spectra stochastic.

5.1. probabilistic pointed cubical sets. We consider here a probabilistic version of the category $\square_{*}$ of pointed cubical sets, modeled on the probabilistic version $\mathcal{P} \mathcal{S}_{*}$ of the category $\mathcal{S}_{*}$ of finite pointed sets.

Definition 5.1. The category $\mathcal{P} \square_{*}$ is obtained as a wreath product of the category $\square_{*}$ and the category of finite sets $\mathcal{F P}$ according to the general procedure of Definition 2.18. Namely, objects of $\mathcal{P} \square_{*}$ are formal convex combinations $\Lambda K=\sum_{i} \lambda_{i} K_{i}$ of objects $K_{i} \in \mathcal{P} \square_{*}$, that is, functors $K_{i}: \mathfrak{C}_{c}^{o p} \rightarrow \mathcal{S}_{*}$. Morphisms in $\mathcal{P} \square_{*}$ between objects $\Lambda K$ and $\Lambda^{\prime} K^{\prime}$ are pairs $\Phi=(S, \eta)$ of a stochastic matrix $S$ with $S \Lambda=\Lambda^{\prime}$ and a collection $\eta=\left\{\eta_{i j, r}\right\}$ with $\eta_{i j, r}: K_{j} \rightarrow K_{i}^{\prime}$ natural transformations chosen with probabilities $\mu_{r}^{i j}$ with $\sum_{r} \mu_{r}^{i j}=S_{i j}$. A probabilistic pointed cubical set with connection is an object in $\mathcal{P} \square_{*}$.

Lemma 5.2. The category $\mathcal{P} \square_{*}$ of probabilistic pointed cubical sets as in Definition 5.1 can be identified with the category of functors

$$
\mathbb{K}: \mathfrak{C}_{c}^{o p} \rightarrow \mathcal{P} \mathcal{S}_{*}
$$

with morphisms given by natural transformations.

Proof. An object $\Lambda K$ in $\mathcal{P} \square_{*}$ defines a fuctor $\mathbb{K}$ with $\mathbb{K}\left(\mathcal{I}^{n}\right)=\sum_{i} \lambda_{i} K_{i}\left(\mathcal{I}^{n}\right)$ in $\mathcal{P} \mathcal{S}_{*}$. Moreover, a morphism $\left(S, \eta=\left\{\eta_{i j, r}\right\}\right)$ in $\mathcal{P} \square_{*}$ defines a natural transformation $\eta$ : $\mathbb{K}=\Lambda K \rightarrow \mathbb{K}^{\prime}=\Lambda^{\prime} K^{\prime}$ with $\left(S, \eta_{n}\right)$ the morphisms in $\mathcal{P} \mathcal{S}_{*} \Lambda^{\prime}=S \Lambda$ and $\eta_{n}=$ $\left\{\eta_{n, i j, r}\right\}: K_{j}\left(\mathcal{I}^{n}\right) \rightarrow K_{i}\left(\mathcal{I}^{n}\right)$. Conversely, a functor $\mathbb{K}: \mathfrak{C}_{c}^{o p} \rightarrow \mathcal{P} \mathcal{S}_{*}$ given, for each $n$, an object $\mathbb{K}\left(\mathcal{I}^{n}\right)=\Lambda^{(n)} K^{(n)} \in \mathcal{P} \mathcal{S}_{*}$. The compatibility with the faces, degeneracies, and connections $\delta_{i}^{a}, \sigma_{i}$, and $\gamma_{i}$ implies that there are morphisms

$$
\Phi^{\delta}=\left(S^{\delta}, \underline{\delta}\right), \quad \Phi^{\sigma}=\left(S^{\sigma}, \underline{\sigma}\right), \quad \Phi^{\gamma}=\left(S^{\gamma}, \underline{\gamma}\right)
$$

with $\Phi^{\delta}=\left(\Phi_{i}^{\delta^{a}}\right)_{u, u^{\prime}}, \Phi^{\sigma}=\left(\Phi_{i}^{\sigma}\right)_{u, u^{\prime}}$, and $\Phi^{\gamma}=\left(\Phi_{i}^{\gamma}\right)_{u, u^{\prime}}$ satisfying the cubical relations. In particular, the relations 4.3), 4.6) imply that the stochastic matrices $S_{\delta_{i}^{a}}, S_{\sigma_{i}}$ and $S_{\gamma_{i}}$ are invertible with stochastic inverses, hence all $\Lambda^{(n)}=\Lambda$ up to permutations and all the morphisms $\underline{\delta}, \underline{\sigma}, \underline{\gamma}$ consist of a single morphism applied with probability one. Thus, up to a permutation of the terms, the $\Phi^{\delta}, \Phi^{\sigma}$ and $\Phi^{\gamma}$ are diagonal, given by the identity on $\Lambda$ and the usual faces, degeneracies, and connections on the underlying pointed cubical sets. Thus, $\mathbb{K}=\Lambda K$ is an object of $\mathcal{P} \square_{*}$ as in Definition 5.1 and the natural transformations are also given by morphisms in $\mathcal{P} \square_{*}$. 
5.2. Stochastic Gamma spaces. We extend then the definition of $\Gamma$-spaces to the probabilistic setting as follows.

Definition 5.3. A probabilistic cubical $\Gamma$-space is a functor $F: \mathcal{P} \mathcal{S}_{*} \rightarrow \mathcal{P} \square_{*}$.

The Segal construction assigns a $\Gamma$-space $F_{\mathcal{C}}$ to a category $\mathcal{C}$ with zero objects and a categorical sum. We show here that the construction extends to obtain a probabilistic $\Gamma$ space.

5.2.1. Stochastic summing functors. Given an object $\Lambda X=\sum_{i} \lambda_{i}\left(X_{i}, x_{i}\right)$ in $\mathcal{P} \mathcal{S}_{*}$, we consider a category $\mathcal{P}(\Lambda X)$ defined as follows.

Definition 5.4. The category $\mathcal{P}(\Lambda X)$ has objects $\Lambda A=\sum_{i} \lambda_{i}\left(A_{i}, x_{i}\right)$ with $\left(A_{i}, x_{i}\right) \in$ $P\left(X_{i}, x_{i}\right)$, where $P\left(X_{i}, x_{i}\right)$ is the category of pointed subsets and inclusions. Morphisms in $\mathcal{P}(\Lambda X)$ are morphisms $\Phi_{A, A^{\prime}}: \Lambda A \rightarrow \Lambda A^{\prime}$ where the stochastic matrix $S$ is the identity and $F_{A, A^{\prime}}=\left\{F_{A_{i}, A_{i}^{\prime}}\right\}$ is a collection of pointed inclusions $F_{A_{i}, A_{i}^{\prime}}:\left(A_{i}, x_{i}\right) \hookrightarrow\left(A_{i}^{\prime}, x_{i}\right)$ applied with probability 1 .

Let $\mathcal{C}$ be a category with zero object and coproduct and let $\mathcal{P C}$ be its probabilistic category as in Definition 2.18.

Definition 5.5. Let $\mathcal{C}$ be a category with zero objects and a categorical sum and let $\Lambda X$ be an object in $\mathcal{P} \mathcal{S}_{*}$. The category $\mathcal{P} \Sigma_{\mathcal{C}}(\Lambda X)$ has objects the summing functors $\Theta: \mathcal{P}(\Lambda X) \rightarrow \mathcal{C}$ and morphisms the natural transformations that are isomorphisms on objects.

Definition 5.6. A summing functor $\Theta: \mathcal{P}(\Lambda X) \rightarrow \mathcal{P C}$ is a functor satisfying $\Theta\left(\sum_{i} \lambda_{i}\left(A_{i}, x_{i}\right)\right)=\sum_{i} \lambda_{i} \Theta_{i}\left(A_{i}, x_{i}\right)$, where $\Theta_{i}: P\left(X_{i}, x_{i}\right) \rightarrow \mathcal{P C}$ are summing functors in $\Sigma_{\mathcal{P C}}\left(X_{i}, x_{i}\right)$. The category $\mathcal{P} \Sigma_{\mathcal{P S}}(\Lambda X)$ has objects the summing functors $\Theta: \mathcal{P}(\Lambda X) \rightarrow \mathcal{P C}$ and morphisms given by natural transformations $\left\{\eta_{i}\right\}$ of the summing functors $\Theta_{i}$ that are isomorphisms on objects.

Lemma 5.7. The the cubical nerve $\mathcal{N}_{\mathfrak{C}}\left(\mathcal{P} \Sigma_{\mathcal{P C}}(\Lambda X)\right)$ can be identified with the probabilistic pointed cubical set $\sum_{i} \lambda_{i} \mathcal{N}_{\mathfrak{C}}\left(\Sigma_{\mathcal{P C}}\left(X_{i}, x_{i}\right)\right)$.

Proof. The cubical nerve is given by $\left(\mathcal{N}_{\mathfrak{C}}\left(\mathcal{P} \Sigma_{\mathcal{P C}}(\Lambda X)\right)_{n}=\operatorname{Fun}\left(\mathcal{I}^{n}, \mathcal{P} \Sigma_{\mathcal{P C}}(\Lambda X)\right)\right.$. Thus, to each vertex $v \in \mathcal{I}^{n}$ one assigns a summing functor $\Theta_{v}=\sum_{i} \lambda_{i} \Theta_{v, i}$ and to each edge $e \in \mathcal{I}^{n}$ one assigns a natural transformation $\eta_{e}=\left\{\eta_{e, i}\right\}$. Thus, a point in $\mathcal{N}_{\mathfrak{C}}\left(\mathcal{P} \Sigma_{\mathcal{P C}}(\Lambda X)\right)$ is specified uniquely by a choice of points in the $\mathcal{N}_{\mathfrak{C}}\left(\Sigma_{\mathcal{P C}}\left(X_{i}, x_{i}\right)\right)$ and viceversa, so that we can identify $\mathcal{N}_{\mathfrak{C}}\left(\mathcal{P} \Sigma_{\mathcal{P C}}(\Lambda X)\right)$ with the data of a probabilistic pointed cubical set given by the formal combination $\sum_{i} \lambda_{i} \mathcal{N}_{\mathfrak{C}}\left(\Sigma_{\mathcal{P C}}\left(X_{i}, x_{i}\right)\right)$.

Lemma 5.8. Given $\mathcal{C}$ a category with zero object and coproduct and $\mathcal{P C}$ its probabilistic category as in Definition 2.18, one obtains an associated probabilistic cubical $\Gamma$-space $F_{\mathcal{P C}}: \mathcal{P} \mathcal{S}_{*} \rightarrow \mathcal{P} \square_{*}$ 
Proof. The functor $F_{\mathcal{P C}}: \mathcal{P} \mathcal{S}_{*} \rightarrow \mathcal{P} \square_{*}$ assigns to an object $\Lambda X=\sum_{i} \lambda_{i}\left(X_{i}, x_{i}\right) \in$ $\operatorname{Obj}\left(\mathcal{P} \mathcal{S}_{*}\right)$ the probabilistic pointed cubical set $\sum_{i} \lambda_{i} \mathcal{N}_{\mathfrak{C}}\left(\Sigma_{\mathcal{P C}}\left(X_{i}, x_{i}\right)\right)$. To a morphism $\Phi=(S, F) \in \operatorname{Mor}_{\mathcal{P S}}\left(\Lambda X, \Lambda^{\prime} X^{\prime}\right)$ with $S \Lambda=\Lambda^{\prime}$ and $F=\left\{F_{i j, r}\right\}$ with probabilities with $\sum_{r} \mu_{r}^{i j}=S_{i j}$, the functor assigns the morphism of probabilistic pointed cubical sets with the same stochastic matrix $S$ and with a collection $\phi_{i j, r}: \mathcal{N}_{\mathfrak{C}}\left(\Sigma_{\mathcal{P C}}\left(X_{j}, x_{j}\right)\right) \rightarrow$ $\mathcal{N}_{\mathfrak{C}}\left(\Sigma_{\mathcal{P C}}\left(X_{i}^{\prime}, x_{i}^{\prime}\right)\right)$ applied with the same probabilities $\mu_{r}^{i j}$.

Remark 5.9. As in the case of ordinary $\Gamma$-spaces, we can extend the functors $F$ : $\mathcal{P} \mathcal{S}_{*} \rightarrow \mathcal{P} \square_{*}$ to endofunctors $F: \mathcal{P} \square_{*} \rightarrow \mathcal{P} \square_{*}$ by an analogous coend construction.

5.3. Smash product of stochastic $\Gamma$-spaces. In the usual setting of pointed sets and pointed simplicial sets, an advantage of using $\Gamma$-spaces to construct spectra lies in the fact that there is a simple construction of a smash product for $\Gamma$-spaces, [25]. The main drawback of restricting attention to spectra obtained from $\Gamma$-spaces is that the construction only gives rise to connective spectra, [30], [33].

The category $\Gamma^{0}$ of finite pointed sets has a smash product functor $\wedge: \Gamma^{0} \times \Gamma^{0} \rightarrow \Gamma^{0}$, with $((X, x),(Y, y) \mapsto(X, x) \wedge(Y, y)=(X \times Y /(X \times\{y\} \cup\{x\} \times Y)$, $)$, which extends to a smash product $K \wedge K^{\prime}$ of arbitrary pointed (simplicial) sets.

The smash product of $\Gamma$-spaces constructed in [25] is obtained by first associating to a pair $F, F^{\prime}: \Gamma^{0} \rightarrow \Delta_{*}$ of $\Gamma$-spaces a bi- $\Gamma$-space $F \tilde{\wedge} F^{\prime}: \Gamma^{0} \times \Gamma^{0} \rightarrow \Delta_{*}$

$$
\left(F \tilde{\wedge} F^{\prime}\right)((X, x),(Y, y))=F(X, x) \wedge F^{\prime}(Y, y)
$$

and then defining

$$
\left(F \wedge F^{\prime}\right)\left((X, x)=\operatorname{colim}_{\left(X_{1}, x_{1}\right) \wedge\left(X_{2}, x_{2}\right) \rightarrow(X, x)}\left(F \wedge F^{\prime}\right)\left(\left(X_{1}, x_{1}\right),\left(X_{2}, x_{2}\right)\right),\right.
$$

where $\left(X_{1}, x_{1}\right) \wedge\left(X_{2}, x_{2}\right)$ is the smash product $\wedge: \Gamma^{0} \times \Gamma^{0} \rightarrow \Gamma^{0}$. It is shown in [25] that, up to natural isomorphism, this smash product is associative and commutative and with unit given by the $\Gamma$-space $\mathbb{S}$, and that the category of $\Gamma$-spaces is symmetric monoidal with respect to this product.

Definition 5.10. Given two probabilistic cubical $\Gamma$-spaces $F, F^{\prime}: \mathcal{P} \mathcal{S}_{*} \rightarrow \mathcal{P} \square_{*}$, we set

$$
\left(F \tilde{\wedge} F^{\prime}\right)\left(\Lambda X, \Lambda^{\prime} Y\right)=F(\Lambda X) \wedge F^{\prime}\left(\Lambda^{\prime} Y\right)
$$

where $\Lambda K \wedge \Lambda^{\prime} K^{\prime}$ in $\mathcal{P} \square_{*}$ is defined as in (4.14)

$$
\Lambda K \wedge \Lambda^{\prime} K^{\prime}=\sum_{i, j} \lambda_{i} \lambda_{j} K_{i} \wedge K_{j}^{\prime},
$$

with $K_{i} \wedge K_{j}^{\prime}$ the smash product in $\square_{*}$. One then defines

$$
\left(F \wedge F^{\prime}\right)(\Lambda X)=\operatorname{colim}_{\Lambda_{1} X_{1} \wedge \Lambda_{2} X_{2} \rightarrow \Lambda X}\left(F \tilde{\wedge} F^{\prime}\right)\left(\left(\Lambda_{1} X_{1}\right),\left(\Lambda_{2} X_{2}\right)\right)
$$

The morphisms $\Lambda_{1} X_{1} \wedge \Lambda_{2} X_{2} \rightarrow \Lambda X$ are of the form $S_{u,\left(a, a^{\prime}\right)}=\lambda_{u}^{-1} S_{u a} S_{u a^{\prime}}^{\prime}$ when $\lambda_{u} \neq 0$ and $S_{u,\left(a, a^{\prime}\right)}=S_{u a}+S_{u a^{\prime}}^{\prime}$ otherwise and $f \wedge f^{\prime}=\left\{f_{u a, r} \wedge f_{u a^{\prime}, r^{\prime}}^{\prime}\right\}$ with probabilities $\lambda_{u}^{-1} \mu_{r}^{u a} \mu_{r^{\prime}}^{u a^{\prime}}$ or $M^{-1} \mu_{r}^{u a}+N^{-1} \mu_{r^{\prime}}^{u a^{\prime}}$, respectively when $\lambda_{u} \neq 0$ or $\lambda_{u}=0$. 


\section{INFORMATION LOSS ON PROBABILISTIC CATEGORIES}

We extend here the information loss functional on $\mathcal{F P}$ discussed in $\$ 3.1$ to probabilistic categories $\mathcal{P C}$ and in particular to the category $\mathcal{P} \mathcal{S}_{*}$ of probabilistic pointed sets and the category $\mathcal{P} \square_{*}$ of probabilistic pointed cubical sets.

6.1. Probabilistic categories and information. Let $\mathcal{C}$ be a category with zero object and coproduct and let $\mathcal{P C}$ be the associated probabilistic category $\mathcal{F} \mathcal{P}<\mathcal{C}$ constructed as in Definition 2.18.

Lemma 6.1. The sets of morphisms $\operatorname{Mor}_{\mathcal{P C}}\left(\Lambda C, \Lambda^{\prime} C^{\prime}\right)$ are convex sets.

Proof. Given $\Phi, \Phi^{\prime} \in \operatorname{Mor}_{\mathcal{P C}}\left(\Lambda C, \Lambda^{\prime} C^{\prime}\right)$ and any $\lambda \in[0,1]$ we obtain a morphism $\lambda \Phi+(1-\lambda) \Phi^{\prime}$ as follows. We have $\Phi=(S, F)$ and $\Phi^{\prime}=\left(S^{\prime}, F^{\prime}\right)$ with $S, S^{\prime}$ stochastic matrices with $S \Lambda=\Lambda^{\prime}$ and $S^{\prime} \Lambda=\Lambda^{\prime}$ and $F=\left\{F_{a b, r}\right\}$ and $F^{\prime}=\left\{F_{a b, r^{\prime}}^{\prime}\right\}$ with respective probabilities $\mu_{r}^{a b}$ and $\nu_{r^{\prime}}^{a b}$ with $\sum_{r} \mu_{r}^{a b}=S_{a b}$ and $\sum_{r^{\prime}} \nu_{r^{\prime}}^{a b}=S_{a b}^{\prime}$. The combination $S_{\lambda}=\lambda S+(1-\lambda) S^{\prime}$ is a stochastic matrix with $S_{\lambda} \Lambda=\Lambda^{\prime}$. We take $F_{\lambda}=\left\{F_{a b, r}\right\} \cup\left\{F_{a b, r^{\prime}}^{\prime}\right\}$ with probabilities $\lambda \mu_{r}^{a b}$ and $(1-\lambda) \nu_{r^{\prime}}^{a b}$. We obtain in this way a morphism $\lambda \Phi+(1-\lambda) \Phi^{\prime}=\left(S_{\lambda}, F_{\lambda}\right) \in \operatorname{Mor}_{\mathcal{P C}}\left(\Lambda C, \Lambda^{\prime} C^{\prime}\right)$.

In the same way, we also have the following forms of convex combinations in the category $\mathcal{P C}$.

Lemma 6.2. Given objects $\Lambda C, \Lambda^{\prime} C^{\prime}$ and $\Sigma C^{\prime \prime}$ in $\mathcal{P C}$, and given $\lambda \in[0,1]$, consider the object

$$
\lambda \Lambda C+(1-\lambda) \Lambda^{\prime} C^{\prime}:=\sum_{i} \lambda \lambda_{i} C_{i}+\sum_{j}(1-\lambda) \lambda_{j}^{\prime} C_{j}^{\prime} .
$$

A choice of morphisms $\Phi \in \operatorname{Mor}_{\mathcal{P C}}\left(\Lambda C, \Sigma C^{\prime \prime}\right)$ and $\Phi^{\prime} \in \operatorname{Mor}_{\mathcal{P C}}\left(\Lambda^{\prime} C^{\prime}, \Sigma C^{\prime \prime}\right)$ determines a morphism

$$
\lambda \Phi+(1-\lambda) \Phi^{\prime} \in \operatorname{Mor}_{\mathcal{P C}}\left(\lambda \Lambda C+(1-\lambda) \Lambda^{\prime} C^{\prime}, \Sigma C^{\prime \prime}\right)
$$

with stochastic matrix $\lambda S+(1-\lambda) S^{\prime}$ and morphisms $\left\{F_{u a, r}\right\} \cup\left\{F_{u a^{\prime}, r^{\prime}}^{\prime}\right\}$ with probabilities $\lambda \mu_{r}^{u a}$ and $(1-\lambda) \mu_{r^{\prime}}^{u a^{\prime}}$. Similarly, given objects $\Lambda C, \Sigma C^{\prime}, \Sigma C^{\prime \prime}$, one can form an object $\lambda \Sigma C^{\prime}+(1-\lambda) \Sigma C^{\prime \prime}$ and morphisms $\lambda \Phi+(1-\lambda) \Phi^{\prime} \in \operatorname{Mor}_{\mathcal{P C}}\left(\Lambda C, \lambda \Sigma C^{\prime}+(1-\lambda) \Sigma C^{\prime \prime}\right)$ with $\lambda S+(1-\lambda) S^{\prime}$ and with $\left\{F_{u a, r}\right\} \cup\left\{F_{u^{\prime} a, r^{\prime}}^{\prime}\right\}$ with probabilities $\lambda \mu_{r}^{u a}$ and $(1-\lambda) \mu_{r^{\prime}}^{u^{\prime} a}$.

Definition 6.3. An information loss functional

$$
\mathcal{H}: \cup_{\Lambda C, \Lambda^{\prime} C^{\prime}} \operatorname{Mor}_{\mathcal{P C}}\left(\Lambda C, \Lambda^{\prime} C^{\prime}\right) \rightarrow \mathbb{R}
$$

is characterized by the properties:

(1) vanishing on isomorphisms: $\mathcal{H}(\Phi)=0$ if $\Phi$ is an isomorphism

(2) additivity under composition: $\mathcal{H}\left(\Phi \circ \Phi^{\prime}\right)=\mathcal{H}(\Phi)+\mathcal{H}\left(\Phi^{\prime}\right)$ 
(3) extensivity under convex combinations:

$$
\mathcal{H}\left(\lambda \Phi+(1-\lambda) \Phi^{\prime}\right)=\lambda \mathcal{H}(\Phi)+(1-\lambda) \mathcal{H}\left(\Phi^{\prime}\right)+\mathcal{H}\left(\hat{1}_{(\lambda, 1-\lambda)}\right)
$$

for objects and morphisms as in (6.1), 6.2), where $\hat{1}_{(\lambda, 1-\lambda)}$ is the unique morphism in $\mathcal{P C}$ from the object $\Lambda 0$, with $\Lambda=(\lambda, 1-\lambda)$ and 0 the zero object of $\mathcal{C}$ to the zero object of $\mathcal{P C}$.

We say that $\mathcal{H}$ is a strong information loss functional if it also satisfies the property

(4) inclusion-exclusion on coproducts:

$$
\mathcal{H}\left(\Phi \amalg_{\mathcal{P C}} \Phi^{\prime}\right)=\mathcal{H}(\Phi)+\mathcal{H}\left(\Phi^{\prime}\right)-\mathcal{H}\left(\widehat{\Sigma C^{\prime \prime}}\right),
$$

for $\Phi \in \operatorname{Mor}\left(\Lambda C, \Sigma C^{\prime \prime}\right), \Phi^{\prime} \in \operatorname{Mor}\left(\Lambda^{\prime} C^{\prime}, \Sigma C^{\prime \prime}\right)$ and $\widehat{\Sigma C^{\prime \prime}}$ the unique morphism from the zero object of $\mathcal{P C}$ to $\Sigma C^{\prime \prime}$.

Remark 6.4. Let $\mathcal{C}$ be a category with zero object and categorical sum, and let $\mathcal{P C}$ be the associated probabilistic category. The category $\mathcal{F} \mathcal{P}$ of finite probabilities embeds in $\mathcal{P C}$ via the functor $\mathcal{J}: \mathcal{F P} \rightarrow \mathcal{P C}$ that maps $\Lambda=\left(\lambda_{i}\right) \mapsto \sum_{i} \lambda_{i} 0_{i}$, a sum of copies of the zero object of $\mathcal{C}$ and morphisms $S$ to $\Phi=(S, 1)$.

Lemma 6.5. An information loss functional on $\mathcal{P C}$ satisfying the first three properties of Definition 6.3 restricts to the embedding of $\mathcal{F P}$ in $\mathcal{P C}$ as $\mathcal{H}(\mathcal{J}(S))=$ $\kappa\left(H\left(\Lambda^{\prime}\right)-H(\Lambda)\right)$, where $H(\Lambda)=-\sum_{i} \lambda_{i} \log \lambda_{i}$ is the Shannon entropy, for some constant $\kappa \neq 0$. The induced $\mathcal{H} \circ \mathcal{J}$ is a strong information loss functional.

Proof. This follows directly from Proposition 3.4, since the restriction to $\mathcal{F} \mathcal{P}$ satisfies the properties of an information loss functional on the category of finite probabilities, which must then be of the form $\mathcal{H}(S)=\kappa\left(H\left(\Lambda^{\prime}\right)-H(\Lambda)\right)$, for some $\kappa \neq 0$. The condition of inclusion-exclusion on coproducts is automatically satisfied by the induced information loss functional on $\mathcal{F P}$, since for a product of statistically independent probabilities the Shannon entropy is additive, $H\left(\Lambda \Lambda^{\prime}\right)=H(\Lambda)+H\left(\Lambda^{\prime}\right)$, hence $\mathcal{H}\left(S \amalg S^{\prime}\right)=\kappa\left(H(\Sigma)-H\left(\Lambda \Lambda^{\prime}\right)\right)=\mathcal{H}(S)+\mathcal{H}\left(S^{\prime}\right)-\kappa H(\Sigma)$, see Corollary 3.5 .

Lemma 6.6. An information loss functional on $\mathcal{P C}$ satisfying the first three properties of Definition 6.3 must be of the form $\mathcal{H}(\Phi)=\tilde{H}\left(\Lambda^{\prime} C^{\prime}\right)-\tilde{H}(\Lambda C)$, for $\Phi \in$ $\operatorname{Mor}_{\mathcal{F P}}\left(\Lambda C, \Lambda^{\prime} C^{\prime}\right)$, with $\tilde{H}: \operatorname{Obj}(\mathcal{P C}) \rightarrow \mathbb{R}$ given by $\tilde{H}(\Lambda C):=-\mathcal{H}\left(\hat{1}_{\Lambda C}\right)$ with $\hat{1}_{\Lambda C}$ the unique morphism in $\mathcal{P C}$ between the object $\Lambda C$ and the zero object of $\mathcal{P C}$.

Proof. The argument is the same as in [2] and in the case of $\mathcal{F} \mathcal{P}$ of Proposition 3.4 , namely the composition $\hat{1}_{\Lambda^{\prime} C^{\prime}} \circ \Phi=\hat{1}_{\Lambda C}$ for all $\Phi \in \operatorname{Mor}_{\mathcal{P C}}\left(\Lambda C, \Lambda^{\prime} C^{\prime}\right)$, hence by additivity under composition $\mathcal{H}(\Phi)=\mathcal{H}\left(\hat{1}_{\Lambda C}\right)-\mathcal{H}\left(\hat{1}_{\Lambda^{\prime} C^{\prime}}\right)$. 
Lemma 6.7. Let $\mathcal{H}$ be an information loss functional on $\mathcal{P C}$ satisfying the first three properties of Definition 6.3, and let $\tilde{H}(\Lambda C):=-\mathcal{H}\left(\hat{1}_{\Lambda C}\right)$ be the associated functional of Lemma 6.6. Then $\tilde{H}$ satisfies the extensivity property

$$
\tilde{H}(\Lambda C)=\kappa H(\Lambda)+\sum_{i} \lambda_{i} \tilde{H}\left(C_{i}\right)=\kappa\left(H(\Lambda)+\sum_{i} \lambda_{i} H\left(C_{i}\right)\right)
$$

where $H(\Lambda)$ is the Shannon entropy and $\tilde{H}=\kappa H: \operatorname{Obj}(\mathcal{C}) \rightarrow \mathbb{R}$ given by $\tilde{H}(C)=$ $-\mathcal{H}\left(\hat{1}_{C}\right)$ with $\hat{1}_{C}$ the unique morphism from $C$ to the zero object of $\mathcal{C}$.

Proof. This property follows from the property of extensivity under convex combinations for information loss functionals of Definition 6.3, which combined with Lemma 6.5 gives

$$
\tilde{H}\left(\lambda \Lambda C+(1-\lambda) \Lambda^{\prime} C^{\prime}\right)=\lambda \tilde{H}(\Lambda C)+(1-\lambda) \tilde{H}\left(\Lambda^{\prime} C^{\prime}\right)+\kappa H(\lambda, 1-\lambda) .
$$

One can then inductively show as in Proposition 3.4 that this implies the extensivity property 6.3.

Lemma 6.8. An information loss functional $\mathcal{H}$ on $\mathcal{P C}$ satisfying the first three properties of Definition 6.3 induces a functional $\mathcal{H}: \operatorname{Mor}_{\mathcal{C}}\left(C, C^{\prime}\right) \rightarrow \mathbb{R}$ given by $\mathcal{H}(f)=\tilde{H}\left(C^{\prime}\right)-\tilde{H}(C)$, with $\tilde{H}: \operatorname{Obj}(\mathcal{C}) \rightarrow \mathbb{R}$ as in Lemma 6. ․ This functional $\mathcal{H}: \operatorname{Mor}_{\mathcal{C}}\left(C, C^{\prime}\right) \rightarrow \mathbb{R}$ satisfies the properties

(1) vanishing on isomorphisms: $\mathcal{H}(f)=0$ if $f$ is an isomorphism

(2) additivity on compositions: $\mathcal{H}\left(f \circ f^{\prime}\right)=\mathcal{H}(f)+\mathcal{H}\left(f^{\prime}\right)$

If $\mathcal{H}$ is a strong information loss functional on $\mathcal{P C}$ satisfying also the fourth condition of Definition 6.3 then $\mathcal{H}: \operatorname{Mor}_{\mathcal{C}}\left(C, C^{\prime}\right) \rightarrow \mathbb{R}$ also satisfies

(3) inclusion-exclusion on coproducts: $\mathcal{H}\left(f \amalg_{\mathcal{C}} f^{\prime}\right)=\mathcal{H}(f)+\mathcal{H}\left(f^{\prime}\right)-\mathcal{H}\left(\hat{C}^{\prime \prime}\right)$, for $f \in \operatorname{Mor}_{\mathcal{C}}\left(C, C^{\prime \prime}\right), f^{\prime} \in \operatorname{Mor}_{\mathcal{C}}\left(C^{\prime}, C^{\prime \prime}\right)$, and $\hat{C}^{\prime \prime}$ the unique morphism in $\mathcal{C}$ from the zero object of $\mathcal{C}$ to $C^{\prime \prime}$.

Proof. The properties follow directly from the properties of the information loss functional $\mathcal{H}: \operatorname{Mor}_{\mathcal{P C}}\left(\Lambda C, \Lambda C^{\prime}\right) \rightarrow \mathbb{R}$ applied to objects given by a single $C, C^{\prime}$ in $\operatorname{Obj}(\mathcal{C})$ with probabilities $\Lambda=1$ and $\Lambda^{\prime}=1$.

In the case of categories $\mathcal{C}$ that also have a product, we can consider a more restrictive kind of information loss functionals on $\mathcal{P C}$, by requiring that the induced information measure on $\mathcal{C}$ of Lemma 6.8 also has a compatibility with products.

Definition 6.9. If the category $\mathcal{C}$ has a product $\otimes$, then a functional $\mathcal{H}(f)=\tilde{H}\left(C^{\prime}\right)-$ $\tilde{H}(C)$ on the morphisms of $\mathcal{C}$, satisfying the properties of Lemma 6.8 is multiplicative if it also has the property that $\tilde{H}: \operatorname{Obj}(\mathcal{C}) \rightarrow \mathbb{R}$ satisfies

$$
\tilde{H}\left(C \otimes C^{\prime}\right)=\tilde{H}(C) \cdot \tilde{H}\left(C^{\prime}\right) \text {. }
$$

Note that the function $\tilde{H}: \operatorname{Obj}(\mathcal{C}) \rightarrow \mathbb{R}$ being multiplicative does not imply any multiplicativity property for $\tilde{H}: \operatorname{Obj}(\mathcal{P C}) \rightarrow \mathbb{R}$, both because the product on $\mathcal{C}$ does not extend to a categorical product on $\mathcal{P C}$, as we previously discussed, and 
because the Shannon entropy $H(\Lambda)$ behaves additively on products of statistically independent measures, while $\tilde{H}(C)$ behaves multiplicatively.

\section{INFORMATION LOSS AND PROBABILISTIC GAMMA SPACES}

We show the existence of interesting information loss functionals on the category $\mathcal{P} \square_{*}$ of probabilistic pointed cubical sets. By the results of Lemma 6.5, Lemma 6.6, and Lemma 6.7, it suffices to construct an information loss functional associated to the objects of $\square_{*}$, namely to the pointed cubical sets given by functors $K: \mathcal{C}_{c}^{o p} \rightarrow \mathcal{S}_{*}$, which satisfies the three properties listed in Lemma 6.8.

The first two properties of Lemma 6.8 are satisfied by a functional of the form $H\left(K^{\prime}\right)-H(K)$ (with $K$ and $K^{\prime}$ the source and target of the morphism) where $H$ is any real valued invariant of cubical (or simplicial) sets. For example, the Euler characteristic, the Betti numbers, the chromatic number, etc. However, the third property of Lemma 6.8, the inclusion-exclusion relation on coproducts restricts the possible invariants that can be used to construct information loss functionals to invariants that behave like an Euler characteristic, in the sense that they satisfy an inclusion-exclusion relation.

The Euler characteristic has an especially nice property among inclusion-exclusion invariants, namely it is (up to a multiplicative constant) the only additive homotopy invariant of finite $\mathrm{CW}$ complexes [8]. Moreover, it is the only homotopy invariant that is determined by a local formula, by adding over vertices a rational contribution given by an alternating sum of an averaged number of $i$-cells that contain the vertex, see [24]. It is unclear, however, whether a local characterization of this kind holds for invariants $\tilde{H}$ arising from information loss functionals.

Lemma 7.1. Let $\mathcal{H}$ be a strong information loss functional on $\mathcal{P C}$ with $\mathcal{C}=\mathcal{S}_{*}$ the category of pointed sets. Then the inclusion-exclusion property for coproducts implies that $\tilde{H}(X, \star)$ satisfies the inclusion-exclusion relation

$$
\tilde{H}(A \cup B, \star)=\tilde{H}(A, \star)+\tilde{H}(B, \star)-\tilde{H}(A \cap B, \star)
$$

for pointed subsets $A, B$ of $(X, \star)$. It also satisfies $\tilde{H}(\star)=0$.

Proof. The relation $\mathcal{H}\left(F \amalg F^{\prime}\right)=\mathcal{H}(F)+\mathcal{H}\left(F^{\prime}\right)-\mathcal{H}(\hat{Y})$ combined with $\mathcal{H}\left(F \amalg F^{\prime}\right)=$ $\tilde{H}(Y)-\tilde{H}\left(X \amalg X^{\prime}\right)$ and $\mathcal{H}(F)=\tilde{H}(Y)-\tilde{H}(X), \mathcal{H}\left(F^{\prime}\right)=\tilde{H}(Y)-\tilde{H}\left(X^{\prime}\right)$ gives additivity on coproducts on objects

$$
\tilde{H}\left(X \amalg X^{\prime}\right)=\tilde{H}(X)+\tilde{H}\left(X^{\prime}\right) .
$$

We write $(A, \star)=\amalg_{a \in A \backslash\{\star\}}(\{a, \star\}, \star)$ and $(B, \star)=\amalg_{b \in B \backslash\{\star\}}(\{b, \star\}, \star)$ so that

$$
\begin{gathered}
\tilde{H}(A \cup B, \star)=\sum_{x \in(A \cup B) \backslash\{\star\}} \tilde{H}(\{x, \star\}, \star) \\
=\sum_{a \in A \backslash\{\star\}} \tilde{H}(\{a, \star\}, \star)+\sum_{b \in B \backslash\{\star\}} \tilde{H}(\{b, \star\}, \star)-\sum_{x \in(A \cap B) \backslash\{\star\}} \tilde{H}(\{x, \star\}, \star)
\end{gathered}
$$




$$
=\tilde{H}(A, \star)+\tilde{H}(B, \star)-\tilde{H}(A \cap B, \star) .
$$

In particular $\tilde{H}(\star)=\tilde{H}((\{\star\}, \star) \amalg(\{\star\}, \star))=\tilde{H}(\star)+\tilde{H}(\star)$, hence $\tilde{H}(\star)=0$.

Corollary 7.2. All the possible functionals $\tilde{H}(A, \star)$ satisfying (7.1) are of the form $\tilde{H}(A, \star)=\kappa(\# A-1)$, for some $\kappa \in \mathbb{R}$.

Proof. Consider the pointed sets of the form $(\{x, \star\}, \star)$. Let $\kappa \in \mathbb{R}$ be the value $\tilde{H}(\{x, \star\}, \star)=\kappa$. For $\kappa=0$ one obtains $\tilde{H} \equiv 0$, in which case the functional (6.3) reduces to just the Shannon entropy, while if $\kappa \neq 0$ then

$$
\tilde{H}(A, \star)=\sum_{a \in A \backslash\{\star\}} \tilde{H}(\{a, \star\}, \star)=\kappa \cdot \#(A \backslash\{\star\}) .
$$

If we drop the strong assumption for the information loss functional $\mathcal{H}$ on $\mathcal{P} \mathcal{S}_{*}$ then we obtain many more possibilities besides multiples the counting of points (reduced Euler characteristic) $\tilde{\chi}(X, x)=\# X-1$.

Lemma 7.3. Let $\mathcal{H}$ be an information loss functional $\mathcal{H}$ on $\mathcal{P} \mathcal{S}_{*}$ satisfying the first three properties of Definition 6.3. Then the induced $\tilde{H}: \operatorname{Obj}\left(\mathcal{S}_{*}\right) \rightarrow \mathbb{R}$ as in Lemma 6.7 is a function $\rho(N)$ of the cardinality $N=\# X-1$. Requiring some additional properties on the behavior on products or coproducts in $\mathcal{S}_{*}$ determines a more restrictive class of functions. For example:

- additivity on coproducts: $\rho(N)=\kappa N$

- multiplicativity on products: $\rho: \mathbb{N} \rightarrow \mathbb{R}_{+}^{*}$ a multiplicative semigroup

- both of the previous properties: $\rho(N)=N$

- additivity on products: $\rho(N)=\kappa \log (N)$ or more generally $\rho(N)=\kappa \log (\sigma(N))$ with $\sigma: \mathbb{N} \rightarrow \mathbb{R}_{+}^{*}$ a multiplicative semigroup

- multiplicativity on coproducts: $\rho(N)=\lambda^{N}$.

Proof. The map $\tilde{H}: \operatorname{Obj}\left(\mathcal{S}_{*}\right) \rightarrow \mathbb{R}$ assigns to a pointed set $(X, x)$ an invariant under isomorphisms, hence a function of the cardinality of $X$. For the listed cases, the first is discussed Corollary 7.2. The product in $\mathcal{S}_{*}$ is the smash product $X \wedge Y=$ $X \times Y /(X \times\{y\} \cup\{x\} \times Y)$ with $\#(X \wedge Y)=N \cdot M$ for $N=\# X-1$ and $M=\# Y-1$, hence multiplicative behavior on product implies $\rho(N M)=\rho(N) \rho(M)$, which means $\rho: \mathbb{N} \rightarrow \mathbb{R}_{+}^{*}$ is a multiplicative semigroup. As a multiplicative semigroup $\mathbb{N}$ is freely generated by the primes, hence a semigroup homomorphism $\rho: \mathbb{N} \rightarrow \mathbb{R}_{+}^{*}$ is determined by specifying a generator $t_{p} \in \mathbb{R}_{+}^{*}$ for each prime $p$ in $\mathbb{N}$. Additivity on coproducts and multiplicativity on products imply that $\tilde{H}$ is the reduced Euler characteristic $\tilde{\chi}(X, x)=\# X-1$. If we require additivity on products $\tilde{H}(X \wedge Y)=$ $\tilde{H}(X)+\tilde{H}(Y)$, we have $\rho(N M)=\rho(N)+\rho(M)$, which is satisfied by functions of the form $\rho(N)=\kappa \log (\sigma(N))$ with $\sigma: \mathbb{N} \rightarrow \mathbb{R}_{+}^{*}$ a multiplicative semigroup. Multiplicative behavior on coproducts is satisfied by exponentiation of any invariant that is additive on coproducts hence by functions of the form $\rho(N)=\lambda^{N}$. 
Lemma 7.4. Let $\mathcal{H}$ be a strong information loss functional on $\mathcal{P C}$ with $\mathcal{C}=\square_{*}$ the category of pointed cubical sets with connection. The inclusion-exclusion property for coproducts implies that $\tilde{H}(K)$ satisfies the inclusion-exclusion relation

$$
\tilde{H}\left(K \cup K^{\prime}\right)=\tilde{H}(K)+\tilde{H}\left(K^{\prime}\right)-\tilde{H}\left(K \cap K^{\prime}\right),
$$

for $K, K^{\prime}$ such that $K \cap K^{\prime}$ is also a pointed cubical sets with connection.

Proof. The inclusion-exclusion property for coproducts on morphisms implies, as above, additivity for coproduct of objects,

$$
\tilde{H}\left(K \amalg_{\square_{*}} K^{\prime}\right)=\tilde{H}(K)+\tilde{H}\left(K^{\prime}\right),
$$

where $K \amalg_{\square_{*}} K^{\prime}=K \vee K^{\prime}$. Then using the previous lemma applied to $\left(K \cup K^{\prime}\right)\left(\mathcal{I}^{n}\right)=$ $K_{n} \cup K_{n}^{\prime} \in \mathcal{S}_{*}$ we obtain the stated inclusion-exclusion relation.

The fact that the invariants $\tilde{H}$ that arise from information loss functionals satisfy an inclusion-exclusion relation is reasonable in the context of information theory. Indeed, mutual information satisfies an inclusion-exclusion relation, hence we can regard the expression (6.3), where $\tilde{H}$ satisfies an inclusion-exclusion relation represented by the additivity $(7.2)$ with respect to the coproduct in $\mathcal{C}$, as a generalization of the inclusionexclusion property of a mutual information measure.

Remark 7.5. Under the hypotheses of Lemma 7.4, if we also know that $\tilde{H}(K)$ is a homotopy invariant, then the additivity on coproducts $\tilde{H}\left(K \vee K^{\prime}\right)=\tilde{H}(K)+\tilde{H}\left(K^{\prime}\right)$ (hence the inclusion-exclusion $(7.3))$ together with the property that $\tilde{H}(\{x, \star\}, \star)=\kappa$ imply that $\tilde{H}(K)=\kappa \cdot \tilde{\chi}(K)$ where $\tilde{\chi}(K)=\chi(K)-1$ is the reduced Euler characteristic. In particular, if $\kappa=1$, this implies that $\tilde{H}(K)=\tilde{\chi}(K)$ is also multiplicative under smash products $\tilde{\chi}\left(K \wedge K^{\prime}\right)=\tilde{\chi}(K) \tilde{\chi}\left(K^{\prime}\right)$, as in Definition 6.9.

The characterization above of the reduced Euler characteristic can also be restated as the characterization as the unique $\mathbb{Z}$ valued function on finite pointed CW complexes that satisfies $\tilde{\chi}(\{x, \star\}, \star)=1$ and $\tilde{\chi}(K)=\tilde{\chi}(A)+\tilde{\chi}(B)$ for any cofiber sequence $A \rightarrow K \rightarrow B$, see Theorem 28.85 of [32].

7.1. Information loss functionals and probabilistic Gamma spaces. Here we consider a fixed information loss functional $\mathcal{H}: \mathcal{P} \square_{*} \rightarrow \mathbb{R}$ with sufficiently good properties and we obtain other information loss functionals on $\mathcal{P} \mathcal{S}_{*}$ and $\mathcal{P} \square_{*}$ (with weaker properties) obtained by precomposing with probabilistic $\Gamma$ spaces $F_{\mathcal{P C}}: \mathcal{P} \mathcal{S}_{*} \rightarrow \mathcal{P} \square_{*}$ associated to probabilistic categories $\mathcal{P C}$.

Lemma 7.6. Let $\mathcal{H}: \mathcal{P} \square_{*} \rightarrow \mathbb{R}$ be an information loss functional satisfying the first three properties of Definition 6.3. Let $F_{\mathcal{P C}}: \mathcal{P S}_{*} \rightarrow \mathcal{P} \square_{*}$ be a probabilistic $\Gamma$-space obtained from a probabilistic category $\mathcal{P C}$ as in Lemma 5.8 , and let $\hat{F}_{\mathcal{P C}}: \mathcal{P} \square_{*} \rightarrow \mathcal{P} \square_{*}$ be its extension to an endofunctor of $\mathcal{P}_{*}$. Then the compositions $\mathcal{H} \circ F_{\mathcal{P C}}$ and $\mathcal{H} \circ \hat{F}_{\mathcal{P C}}$ are also information loss functionals, respectively on $\mathcal{P} \mathcal{S}_{*}$ and $\mathcal{P} \square_{*}$, satisfying the first three properties of Definition 6.3. 
Proof. By functoriality $F_{\mathcal{P C}}$ maps compositions of morphisms to compositions, hence the additivity of $\mathcal{H}$ under composition is preserved by precomposing with $F_{\mathcal{P C}}$. Vanishing on isomorphisms is also preserved for the same reason. Extensivity under convex combination is preserved because, as seen in Lemma 5.7, the functor $F_{\mathcal{P C}}$ assigns to an object $\Lambda X=\sum_{i} \lambda_{i}\left(X_{i}, x_{i}\right)$ in $\mathcal{P} \mathcal{S}_{*}$ the object in $\mathcal{P} \square_{*}$ given by the combination $\sum_{i} \lambda_{i} K_{i}$ with $K_{i}=\mathcal{N}_{\mathfrak{C}}\left(\Sigma_{\mathcal{P C}}\left(X_{i}, x_{i}\right)\right)$. Thus, in particular it satisfies

$$
F_{\mathcal{P C}}\left(\lambda \Lambda X+(1-\lambda) \Lambda^{\prime} X^{\prime}\right)=\lambda F_{\mathcal{P C}}(\Lambda X)+(1-\lambda) F_{\mathcal{P C}}\left(\Lambda^{\prime} X^{\prime}\right),
$$

and the extensivity of $\mathcal{H}$ then gives

$\mathcal{H}\left(\lambda F_{\mathcal{P C}}(\Lambda X)+(1-\lambda) F_{\mathcal{P C}}\left(\Lambda^{\prime} X^{\prime}\right)\right)=\lambda \mathcal{H}\left(F_{\mathcal{P C}}(\Lambda X)\right)+(1-\lambda) \mathcal{H}\left(F_{\mathcal{P C}}\left(\Lambda^{\prime} X^{\prime}\right)\right)+\mathcal{H}\left(\hat{1}_{(\lambda, 1-\lambda)}\right)$.

Remark 7.7. The compositions $\mathcal{H} \circ F_{\mathcal{P C}}$ and $\mathcal{H} \circ \hat{F}_{\mathcal{P C}}$ in general do not satisfy the strong condition of Definition 6.3, even if $\mathcal{H}$ is a strong information loss functional on $\mathcal{P} \square_{*}$. This can be seen in the case of $\mathcal{C}=\mathcal{S}_{*}$. The description of the pointed cubical sets $\mathcal{N}_{\mathfrak{C}}\left(\Sigma_{\mathcal{P} \mathcal{S}_{*}}(X, x)\right)$ in Proposition 4.3 and Proposition 4.4 shows that $\mathcal{N}_{\mathfrak{C}}\left(\Sigma_{\mathcal{P S}}((X, x) \vee(Y, y))\right.$ is not additive in $(X, x)$ and $(Y, y)$ hence even if $\mathcal{H}$ satisfies the strong condition of Definition 6.3 the composition $\mathcal{H} \circ F_{\mathcal{P}}$ does not.

Let $\mathcal{H}: \mathcal{P} \square_{*} \rightarrow \mathbb{R}$ be an information loss functional satisfying the first three properties of Definition 6.3, given by a difference of invariants of target and source objects of the form

$$
\tilde{H}(\Lambda K)=H(\Lambda)+\sum_{i} \lambda_{i} H\left(K_{i}\right), \quad \text { with } \quad H(K)=\log \tilde{\chi}(K),
$$

with $H(\Lambda)$ the Shannon entropy and with $\tilde{\chi}(K)$ the reduced Euler characteristic. Clearly this information loss functional does not satisfy the inclusion-exclusion property of the strong condition. It satisfied instead an additivity property on products

$$
H\left(K \wedge K^{\prime}\right)=H(K)+H\left(K^{\prime}\right),
$$

which follows from the multiplicative property of the reduced Euler characteristic. It also satisfies homotopy invariance, since it factors through the reduced Euler characteristic.

Consider an information loss functional as above, with (7.4), so that it satisfies the additivity property $(7.5)$. Consider probabilistic $\Gamma$-spaces $F: \mathcal{P} \mathcal{S}_{*} \rightarrow \mathcal{P} \square_{*}$ of the form

$$
F(\Lambda X)=\Lambda^{\prime} K^{\prime} \wedge F_{\mathcal{P S}}(\Lambda X),
$$

for $\Lambda^{\prime} K^{\prime}$ a given stochastic pointed cubical set in $\mathcal{P} \square_{*}$. These generalize in our probabilistic setting the classical $\Gamma$-spaces $F: \Gamma^{0} \rightarrow \Delta_{*}$ of the form $F(X)=K \wedge F_{\Gamma^{0}}(X)$, with $F_{\Gamma^{0}}: \Gamma^{0} \hookrightarrow \Delta_{*}$, whose associated spectrum, obtained via the Segal construction, 
is the suspension spectrum of the simplicial set $K$. These probabilistic $\Gamma$-spaces represent a product of two statistically independent systems, $\Lambda^{\prime} K^{\prime}$ and $F_{\mathcal{P S}}(\Lambda X)$ and the chosen information measure accordingly splits additively

$$
\tilde{H}(F(\Lambda X))=\tilde{H}\left(\Lambda^{\prime} K^{\prime}\right)+\tilde{H}\left(F_{\mathcal{P} \mathcal{S}_{*}}(\Lambda X)\right) .
$$

Conversely, any probabilistic $\Gamma$-space $F: \mathcal{P} \mathcal{S}_{*} \rightarrow \mathcal{P} \square_{*}$ with the property that $H(F(\Lambda X))=\alpha+H\left(F_{\mathcal{P S}}(\Lambda X)\right)$ for some fixed $\alpha \in \mathbb{R}$ independent of $X$ and $\Lambda$ and for all $\Lambda X \in \mathcal{P} \mathcal{S}_{*}$ should be regarded from the information point of view as equivalent to a product of two statistically independent systems, one of which is the basic $F_{\mathcal{P} \mathcal{S}_{*}}(\Lambda X)$.

\section{Quantum Information and Categories}

The probabilistic category $\mathcal{P C}$ associated to a category $\mathcal{C}$ with zero object and sum can be seen as a wreath product of the category $\mathcal{C}$ and the category $\mathcal{F} \mathcal{P}$ of finite classical probabilities, hence as a probabilistic version of the category $\mathcal{C}$, in the context of classical probability. We consider here a similar approach that associates to a category $\mathcal{C}$ with zero object and sum a probabilistic category $\mathcal{Q C}$ based on quantum rather than classical probability.

8.1. The category of quantum probabilities. We assign to a finite set $X$ a Hilbert space $\mathcal{H}_{X}=\oplus_{x \in X} \mathbb{C}_{x}$ with $\mathbb{C}_{x}$ a one-dimensional space at the site $x \in X$. More generally, we can replace the $\mathbb{C}_{x}$ with copies of a fixed finite dimensional Hilbert space $\mathcal{V}$ of a fixed dimension, which represents the internal degrees of freedom at the site $x \in X$.

Definition 8.1. The category $\mathcal{F} \mathcal{Q}$ of finite quantum probabilities has objects given by pairs pairs $\left(X, \rho_{X}\right)$ of a finite set $X$ and a density matrix $\rho_{X}$ on the finite dimensional Hilbert space $\mathcal{H}_{X}$, that is, a linear operator on $\mathcal{H}_{X}$ satisfying $\rho_{X}^{*}=\rho_{X}, \rho_{X} \geq 0$, and $\operatorname{Tr}\left(\rho_{X}\right)=1$. The morphisms $\operatorname{Mor}_{\mathcal{F} \mathcal{Q}}\left(\left(X, \rho_{X}\right),\left(Y, \rho_{Y}\right)\right)$ are given by quantum channels $\Phi$, that is, completely positive trace preserving maps with $\Phi\left(\rho_{X}\right)=\rho_{Y}$.

Quantum channels $\Phi$ can always be written (non-uniquely) in Kraus form as

$$
\Phi(\rho)=\sum_{i} A_{i} \rho A_{i}^{*}, \quad \text { with } \quad \sum_{i} A_{i}^{*} A_{i}=1 .
$$

One can also represent completely positive trace preserving maps with $\Phi\left(\rho_{X}\right)=\rho_{Y}$ through the associated stochastic Choi matrix $S_{\Phi}$ with

$$
\left(\rho_{Y}\right)_{i j}=\sum_{a, b}\left(S_{\Phi}\right)_{a b}\left(\rho_{X}\right)_{a b}
$$

Kraus representations can be obtained from factorizations $S_{\Phi}=A A^{*}$. 
8.2. Quantum probabilistic categories $\mathcal{Q C}$. As in the case of classical probability, given a category $\mathcal{C}$ with zero object and sum, we construct a probabilistic version $\mathcal{Q C}$ that maintains the same properties, but in this case based on the quantum probabilities of $\mathcal{F} \mathcal{Q}$ rather than on the classical probabilities of $\mathcal{F P}$.

Definition 8.2. The category $\mathcal{Q C}$ has objects given by $\rho C=\left(\left(C_{a}, C_{b}\right), \rho_{a b}\right)_{a b}$, where $\left(C_{a}, C_{b}\right)$ is a finite collection of pairs of objects in $\mathcal{C}$ indexed over a finite set $a, b=$ $1, \ldots, N$ and $\rho=\left(\rho_{a b}\right)$ is an $N \times N$ density matrix. For $\rho C=\left(\left(C_{a}, C_{b}\right), \rho_{a b}\right)$ and $\rho^{\prime} C^{\prime}=\left(\left(C_{i}^{\prime}, C_{j}^{\prime}\right), \rho_{i j}^{\prime}\right)$, the morphisms $\Xi \in \operatorname{Mor}_{\mathcal{Q C}}\left(\rho C, \rho^{\prime} C^{\prime}\right)$ are given by a finite collection

$$
\left.\Xi=\left\{\left(\phi_{a i, r}, \psi_{b j, r}\right)\right\},\left(S_{\Phi_{r}}\right)_{i j}\right\}
$$

where $\sum_{r} S_{\Phi_{r}}=S_{\Phi}$ is the Choi matrix of a quantum channel $\Phi$ with $\Phi(\rho)=\rho^{\prime}$. The composition of morphisms $\Xi^{\prime} \circ \Xi$ is given by the collection

$$
\Xi^{\prime} \circ \Xi=\left\{\left(\phi_{u a, r^{\prime}} \circ \phi_{a i, r}, \psi_{v b, r^{\prime}} \circ \psi_{b j, r}\right),\left(S_{\Phi_{r}}\right)_{i b}\left(S_{\Phi_{r^{\prime}}^{\prime}}\right)_{u v} \underset{u v}{ }\right\}
$$

which satisfies $\sum_{r, r^{\prime}, i, j}\left(S_{\Phi_{r}}\right)_{i j}^{a b}\left(S_{\Phi_{r^{\prime}}}\right)_{u v}^{i j}=\sum_{i, j}\left(S_{\Phi}\right)_{i j}^{a b}\left(S_{\Phi^{\prime}}\right)_{u v}=\left(S_{\Phi^{\prime} \circ \Phi}\right)_{u b}^{a b}$

Remark 8.3. The notation for the objects of $\mathcal{Q C}$ in the form $\rho C=\left(\left(C_{a}, C_{b}\right), \rho_{a b}\right)_{a b}$, for $a, b=1, \ldots, N$, includes the case where $N=1$. In this case the objects are just single objects $C \in \operatorname{Obj}(\mathcal{C})$ with weight $\rho=1$ and morphisms in $\mathcal{Q C}$ between two objects of this form are morphisms in $\mathcal{C}$. This embeds the category $\mathcal{C}$ into its quantum probability version $\mathcal{Q C}$, as in the case of the classical probabilities.

Remark 8.4. As usual in quantum information, one interprets the off-diagonal terms $\rho_{i j}$ of a density matrix $\rho$ as describing the interference between the amplitudes of the $i$-th and $j$-th state, hence a measure of coherence of the mixed state. Thus, the objects $\rho C$ of the category $\mathcal{Q C}$ have an assigned amount of coherence of pairs of objects in $\mathcal{C}$, described by the coefficients $\rho_{i j}$ of a density matrix. The morphisms in $\mathcal{Q C}$ also correspond to pairs of morphisms in $\mathcal{C}$ with assigned coherence, but also transform the density matrix of the source to that of the target through a quantum channel obtained as the combined coherence measures of all the pairs in the collection.

Proposition 8.5. Let $\mathcal{C}$ be a category with zero object and sum. Then the category $\mathcal{Q C}$ of Definition 8.2 also has a zero object and categorical sum. The zero object is given by the pair $(0,1)$ with 0 the zero object of $\mathcal{C}$ with $\rho=1$ and the coproduct is of the form

$$
\rho C \amalg \rho^{\prime} C^{\prime}=\left(C_{i} \amalg_{\mathcal{C}} C_{j}^{\prime}, \rho \otimes \rho^{\prime}\right) .
$$

Proof. The argument is analogous to the case of classical probabilities that we discussed previously. The zero object of $\mathcal{Q C}$ is given by the pair $(0,1)$ with 0 the zero object of $\mathcal{C}$ and $\rho=1$. There is a unique morphism in $\mathcal{Q C}$ from $(0,1)$ to an object $\rho C=\left(\left(C_{i}, C_{j}\right), \rho_{i j}\right)$ given by the unique morphisms $0 \rightarrow C_{i}$ in $\mathcal{C}$ and $\underset{0}{i j}=\rho_{i j}$. 
The unique morphism from an object $\rho C=\left(\left(C_{i}, C_{j}\right), \rho_{i j}\right)$ to the zero object similarly consists of the unique morphisms $C_{i} \rightarrow 0$ in $\mathcal{C}$ and $\Phi_{i j}=\delta_{i j}$, which gives $\sum_{i j} \Phi_{i j} \rho_{i j}=\operatorname{Tr}(\rho)=1$. The universal property of the coproduct is satisfied with maps ${ }^{i j}$

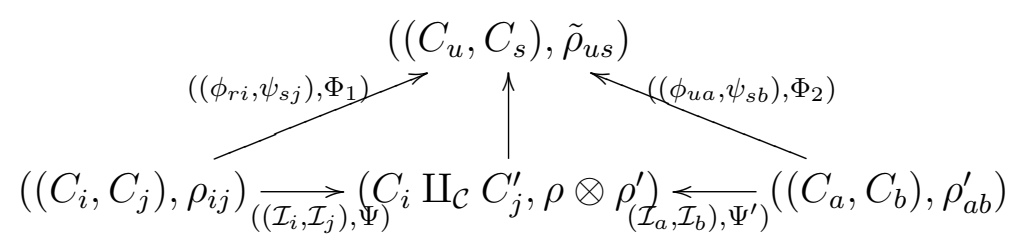

where $\mathcal{I}_{i}: C_{i} \rightarrow C_{i} \amalg_{\mathcal{C}} C_{j}^{\prime}$ are the maps of the universal property of the coproduct in $\mathcal{C}$ and the maps $\Psi \rho=\rho \otimes \rho^{\prime}$ and $\Psi^{\prime} \rho^{\prime}=\rho \otimes \rho^{\prime}$ are given by

$$
\Psi_{\left(i^{\prime} j^{\prime}\right),(a b)}^{i j}=\delta_{i i^{\prime}} \delta_{j j^{\prime}} \rho_{a b}^{\prime} \quad \text { and } \quad \Psi_{(i j),\left(a^{\prime} b^{\prime}\right)}^{a b}=\delta_{a a^{\prime}} \delta_{b b^{\prime}} \rho_{i j}
$$

The map $\rho C \amalg \rho^{\prime} C^{\prime} \rightarrow \tilde{\rho} \tilde{C}$ that makes the diagram commute is then given, at the level of the quantum channels, by

$$
\tilde{\Phi}_{(i j),(a b)}^{u s}=\tilde{\rho}_{u s}^{-1}\left(\Phi_{1}\right)_{i j}^{u s}\left(\Phi_{2}\right)_{a b}^{u s}
$$

when the entry $\tilde{\rho}_{u s} \neq 0$ and

$$
\tilde{\Phi}_{(i j),(a b)}^{u s}=\left(\Phi_{1}\right)_{i j}^{u s} \delta_{a b}+\left(\Phi_{2}\right)_{a b}^{u s} \delta_{i j}
$$

when the matrix entry $\tilde{\rho}_{u s}=0$. Indeed this gives for $\tilde{\rho}_{u s} \neq 0$

$$
\sum_{\left(i^{\prime}, j^{\prime}\right),(a, b)} \tilde{\Phi}_{\left(i^{\prime} j^{\prime}\right),(a b)}^{u s} \Psi_{\left(i^{\prime} j^{\prime}\right),(a b)}=\left(\sum_{i^{\prime}, j^{\prime}}\left(\Phi_{1}\right)_{i^{\prime} j^{\prime}} \delta_{i i^{\prime}} \delta_{j j^{\prime}}\right) \cdot \tilde{\rho}_{u s}^{-1}\left(\sum_{a, b}\left(\Phi_{2}\right)_{a b} \rho_{a b}^{\prime}\right)=\left(\Phi_{1}\right)_{i j s}
$$

and for $\tilde{\rho}_{u s}=0$ it gives

$$
\begin{gathered}
\sum_{i^{\prime}, j^{\prime}, a, b}\left(\left(\Phi_{1}\right)_{i^{\prime} j^{\prime} j^{\prime}} \delta_{a b} \delta_{i i^{\prime}} \delta_{j j^{\prime}} \rho_{a b}^{\prime}+\left(\Phi_{2}\right)_{a b} \delta_{i j} \delta_{i i^{\prime}} \delta_{j j^{\prime}} \rho_{a b}^{\prime}\right) \\
=\left(\sum_{a} \rho_{a a}^{\prime}\right)\left(\Phi_{1}\right)_{i j}^{u s}+\sum_{a b}\left(\Phi_{2}\right)_{a b} \rho_{a b}^{\prime} \delta_{i j}
\end{gathered}
$$

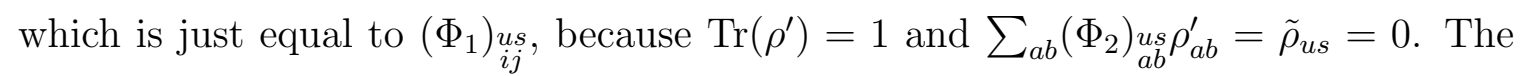
case of composition with $\Psi^{\prime}$ is analogous. At the level of the morphisms, one considers the coproducts $\left(\phi_{u i, r} \amalg_{\mathcal{C}} \phi_{u a, r^{\prime}}, \psi_{s j, r} \amalg_{\mathcal{C}} \psi_{s b, r^{\prime}}\right)$, with $r=1, \ldots, N$ and $r^{\prime}=1, \ldots, M$, in $\mathcal{C}$ weighted with

$$
\begin{gathered}
\tilde{\rho}_{u s}^{-1}\left(\Phi_{1, r}\right)_{i j}^{u s}\left(\Phi_{2, r^{\prime}}\right)_{a s}^{u s}, \quad \text { for } \quad \tilde{\rho}_{u s} \neq 0 \\
\left(\Phi_{1, r}\right)_{i j}^{u s} \frac{\delta_{a b}}{M}+\left(\Phi_{2, r^{\prime}}\right)_{a b}^{u s} \frac{\delta_{i j}}{N}, \quad \text { for } \quad \tilde{\rho}_{u s}=0 .
\end{gathered}
$$

The rest of the argument is analogous to Theorem 2.17 .

Remark 8.6. Note that, as in the case of classical probabilities, the coproduct induced by $(8.2)$ on the category $\mathcal{F} \mathcal{Q}$ of finite quantum probabilities is just the product of independent systems $\rho \amalg_{\mathcal{F} \mathcal{Q}} \rho^{\prime}=\rho \otimes \rho^{\prime}$. 
We can identify a decoherence subcategory of $\mathcal{Q C}$ that corresponds to the case of mixed states with diagonal density matrices (in a fixed basis). This can be described the following category.

Definition 8.7. The decoherence subcategory $\mathbb{P C}$ has objects given by pairs $(C, z)=$ $\left(\left(C_{1}, \ldots, C_{n}\right),\left(z_{1}: \cdots: z_{n}\right)\right)$ with $C_{i} \in \operatorname{Obj}(\mathcal{C})$ and $z=\left(z_{1}: \cdots: z_{n}\right) \in \mathbb{P}^{n-1}(\mathbb{C})$ with morphisms given by a morphism $\Phi: \mathbb{P}^{n-1} \rightarrow \mathbb{P}^{m-1}$ induced by a linear map $\tilde{\Phi}: \mathbb{C}^{n} \rightarrow \mathbb{C}^{m}$ up to scalars with $\Phi z=z^{\prime}$ and a collection $\left\{\left(\tilde{\phi}_{j i, r}: C_{i} \rightarrow C_{j}^{\prime}, \tilde{\Phi}_{r}\right)\right\}$ with $\sum_{r} \tilde{\Phi}_{r}=\tilde{\Phi}$. The coproduct is given by $(C, z) \amalg\left(C^{\prime}, z^{\prime}\right)=\left(\left(C_{i} \amalg C_{j}\right)_{i j}, \alpha_{n, m}\left(z, z^{\prime}\right)\right)$ where $\alpha_{n, m}: \mathbb{P}^{n-1} \times \mathbb{P}^{m-1} \rightarrow \mathbb{P}^{n m-1}$ is the Segre embedding.

In particular, in this case one can interpret the objects $(C, z)$ as a superposition of the objects $C_{i}$ where the probability of observing $C_{i}$ is $\left|z_{i}\right|^{2}$.

8.3. A variant: categories of arrows. A variant on the construction of the categories $\mathcal{Q C}$ considered in the previous section can be obtained by working with arrows of $\mathcal{C}$ instead of pairs of objects in $\mathcal{C}$. We illustrate this version of the construction here. The results in the following sections apply to both the categories $\mathcal{Q C}$ constructed above and the categories $\mathcal{Q} \mathcal{A C}$ constructed here.

First we associate to a category $\mathcal{C}$ with zero object and sum another category $\mathcal{A C}$ with objects the morphisms of $\mathcal{C}$.

Definition 8.8. The category $\mathcal{A C}$ has objects $\phi_{C, C^{\prime}}$ given by elements of $\operatorname{Mor}_{\mathcal{C}}\left(C, C^{\prime}\right)$ for arbitrary $C, C^{\prime} \in \operatorname{Obj}(\mathcal{C})$ and morphisms $L \in \operatorname{Mor}_{\mathcal{A C}}\left(\phi_{C, C^{\prime}}, \phi_{A, A^{\prime}}\right)$ given by pairs $L=\left(L_{1}, L_{2}\right)$ with $L_{1} \in \operatorname{Mor}_{\mathcal{C}}(C, A)$ and $L_{2} \in \operatorname{Mor}_{\mathcal{C}}\left(C^{\prime}, A^{\prime}\right)$ such that the diagram commutes

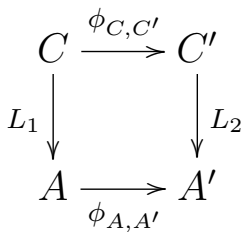

Lemma 8.9. If the category $\mathcal{C}$ has zero object and categorical sum then the category $\mathcal{A C}$ also does. The zero object of $\mathcal{A C}$ is the identity morphism $1_{0}$ of the zero object of $\mathcal{C}$ and the coproduct $\phi_{C, C^{\prime}} \amalg_{\mathcal{A C}} \phi_{A, A^{\prime}}$ is given by the unique morphism $\phi_{C \amalg A, C^{\prime} \amalg A^{\prime}}$ : $C \amalg_{\mathcal{C}} A \rightarrow C^{\prime} \amalg_{\mathcal{C}} A^{\prime}$ determined by the morphisms $\phi_{C, C^{\prime}}$ and $\phi_{A, A^{\prime}}$.

Proof. There is a unique morphism $L=\left(L_{1}, L_{2}\right)$ from any $\phi_{C, C^{\prime}}$ to the zero object $1_{0}$ with $L_{1}$ the unique morphism in $\mathcal{C}$ from $C$ to the zero object and $L_{2}$ the unique morphism in $\mathcal{C}$ from $C^{\prime}$ to the zero object. Similarly, there is a unique morphism from the zero object $1_{0}$ to any $\phi_{C, C^{\prime}}$ with $L_{1}$ the unique morphism in $\mathcal{C}$ from the zero object to $C$ and $L_{2}$ the unique morphism in $\mathcal{C}$ from the zero object to $C^{\prime}$, hence $1_{0}$ is a zero object in $\mathcal{A C}$. Consider the morphisms $L_{C}: C \rightarrow C \amalg_{\mathcal{C}} A$ and $L_{A}: A \rightarrow C \amalg_{\mathcal{C}} A$ in $\mathcal{C}$ that satisfy the universal property of the coproduct in $\mathcal{C}$. Similarly, consider $L_{C^{\prime}}: C^{\prime} \rightarrow C^{\prime} \amalg_{\mathcal{C}} A^{\prime}$ and $L_{A^{\prime}}: A^{\prime} \rightarrow C^{\prime} \amalg_{\mathcal{C}} A^{\prime}$. Given morphisms $\phi_{C, C^{\prime}}$ and $\phi_{A, A^{\prime}}$, by 
the universal property of the coproduct in $\mathcal{C}$ there is a unique morphism from $C \amalg_{\mathcal{C}} A$ to $C^{\prime} \amalg_{\mathcal{C}} A^{\prime}$ such that the diagram commutes

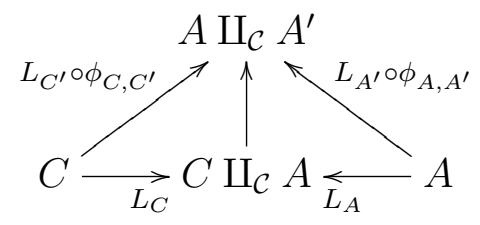

We show that the morphism $\phi_{C, C^{\prime}} \amalg_{\mathcal{A C}} \phi_{A, A^{\prime}}: C \amalg_{\mathcal{C}} A \rightarrow C^{\prime} \amalg_{\mathcal{C}} A^{\prime}$ obtained in this way is the coproduct in $\mathcal{A C}$ by showing that is satisfies the universal property. Suppose given morphisms in $\mathcal{A C}$

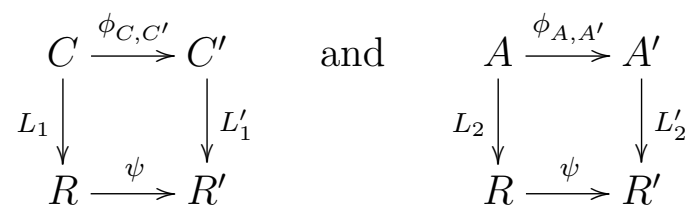

By the universal property of the coproduct in $\mathcal{C}$ there are unique morphisms $L$ : $C \amalg_{\mathcal{C}} A \rightarrow R$ and $L^{\prime}: C^{\prime} \amalg_{\mathcal{C}} A^{\prime} \rightarrow R^{\prime}$ such that $L \circ L_{C}=L_{1}$ and $L \circ L_{A}=L_{2}$ and $L^{\prime} \circ L_{C^{\prime}}=L_{1}^{\prime}$ and $L^{\prime} \circ L_{A^{\prime}}=L_{2}^{\prime}$. The diagram

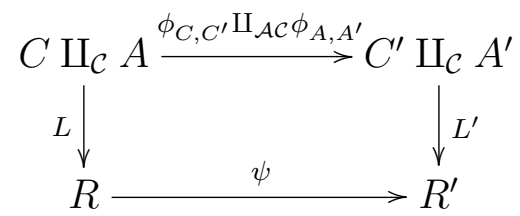

commutes because both $\psi \circ L$ and $L \circ\left(\phi_{C, C^{\prime}} \amalg_{\mathcal{A C}} \phi_{A, A^{\prime}}\right)$ have the property that they give a vertical arrow that makes the following diagram commutative

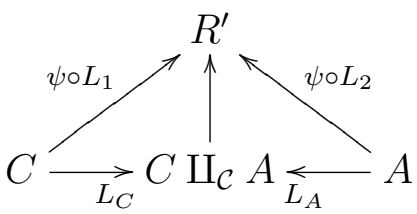

as one can see by replacing $\psi \circ L_{1}=L_{1}^{\prime} \circ \phi_{C, C^{\prime}}$ and $\psi \circ L_{2}=L_{2}^{\prime} \circ \phi_{A, A^{\prime}}$. By the universal property of the coproduct in $\mathcal{C}$ there is a unique morphism with this property, hence $\psi \circ L=L \circ\left(\phi_{C, C^{\prime}} \amalg_{\mathcal{A C}} \phi_{A, A^{\prime}}\right)$. Thus, $\left(L, L^{\prime}\right): \phi_{C, C^{\prime}} \amalg_{\mathcal{A C}} \phi_{A, A^{\prime}} \rightarrow \psi$ constructed in this way is a morphism in $\mathcal{A C}$, and it is the unique morphism such that $\left(L, L^{\prime}\right) \circ\left(L_{C}, L_{A}\right)=\left(L_{1}, L_{2}\right)$ and $\left(L, L^{\prime}\right) \circ\left(L_{C^{\prime}}, L_{A^{\prime}}\right)=\left(L_{1}^{\prime}, L_{2}^{\prime}\right)$. This shows that the coproduct in $\mathcal{A C}$ satisfies the universal property.

Then we associate to the category of arrows $\mathcal{A C}$ a category $\mathcal{Q} \mathcal{A C}$, which can be seen as a wreath product of $\mathcal{A C}$ and the category of finite quantum probabilities $\mathcal{F} \mathcal{Q}$, defined as follows.

Definition 8.10. The category $\mathcal{Q} \mathcal{A C}$ has objects $\rho \phi=\left\{\phi_{i j}, \rho_{i j}\right\}$ given by collections of morphisms $\phi_{i j}: C_{i} \rightarrow C_{j}$ in $\mathcal{C}$, for $i, j=1, \ldots, N$ for any $N \in \mathbb{N}$, together with an $N \times N$ density matrix $\rho=\left(\rho_{i j}\right)$. Morphisms $\operatorname{Mor}_{\mathcal{Q A C}}\left(\rho \phi, \rho^{\prime} \phi^{\prime}\right)$, with $\phi=\left(\phi_{i j}\right)$ and 
$\phi^{\prime}=\left(\phi_{a b}^{\prime}\right)$ are pairs $(L, \Phi)$ of a quantum channel $\Phi(\rho)=\rho^{\prime}$, with Choi matrix $\left(S_{\Phi}\right)_{i j}$

and a finite collection $L=\left\{\left(\underset{a b}{L_{i j}, r},\left(S_{\Phi_{r}}\right)_{i j}\right)\right\}$ of morphisms $\left.\underset{a b}{L_{i j}, r}, \phi_{i j}\right) \rightarrow \phi_{a b}^{\prime}$ in $\mathcal{A C}$ with associated $S_{\Phi_{r}}$ satisfying $\sum_{r}^{a b} S_{\Phi_{r}}=\stackrel{a b}{S_{\Phi}}$.

\section{Quantum Information and Gamma Spaces}

As in the case of classical information discussed earlier, we can construct a $\Gamma$ space $F_{\mathcal{Q C}}: \Gamma^{0} \rightarrow \square_{*}$ associated to a quantum probabilistic category $\mathcal{Q C}$ obtained as in the previous section. We can then consider the associated probabilistic $\Gamma$-space $F_{\mathcal{Q C}}: \mathcal{P} \mathcal{S}_{*} \rightarrow \mathcal{P} \square_{*}$. We consider explicitly the case where the underlying category $\mathcal{C}$ is given by the category of pointed sets $\mathcal{S}_{*}$.

9.1. Summing functors of quantum pointed sets. We refer here to the category $\mathcal{Q C}$ with $\mathcal{C}=\mathcal{S}_{*}$ the category of finite pointed sets as "quantum pointed sets". We consider here the associated category $\Sigma_{\mathcal{Q} \mathcal{S}_{*}}(X)$ of summing functors $\Theta: P(X) \rightarrow$ $\mathcal{Q S}_{*}$, for a pointed set $(X, \star)$, with $P(X)$ the category of pointed subsets $(A, \star)$ with morphisms given by inclusions.

Theorem 9.1. An object $\Theta$ in the category of summing functors $\Sigma_{\mathcal{Q S}}(X)$ is completely specified by the choice of a point $\alpha=\left\{\alpha_{x}\right\}_{x \in X \backslash\{\star\}} \in \mathcal{I}^{N}$, with $\# X=N+1$, and, for each choice of $\alpha$, a set of complex numbers $\theta=\left\{\theta_{x}\right\}_{x \in X \backslash\{\star\}}$ contained in the annuli

$$
\theta_{x} \in \mathcal{A}_{x}=\left\{z \in \mathbb{C}: \alpha_{x}\left(1-\alpha_{x}\right)-\frac{1}{4} \leq|z|^{2} \leq \alpha_{x}\left(1-\alpha_{x}\right)\right\},
$$

or disks $\left\{|z|^{2} \leq \alpha_{x}\left(1-\alpha_{x}\right)\right\}$ if $\alpha_{x}\left(1-\alpha_{x}\right) \leq 1 / 4$. The summing functor then maps $\Theta_{\alpha, \theta}(A)=\rho_{A} C_{A}$ where $\rho_{A} C_{A}$ consists of a collection of $2^{N_{A}} \times 2^{N_{A}}$ pairs of pointed sets of cardinality $N_{A}+1=\# A$ with $\rho=\left(\rho_{i j}\right)$ the $2^{N_{A}} \times 2^{N_{A}}$ density matrix with entries given by the sequences $\left(t_{1}, \ldots, t_{N_{A}}\right)$ with $t_{a} \in\left\{\alpha_{a}, 1-\alpha_{a}, \theta_{a}, \bar{\theta}_{a}\right\}$ for $a \in A \backslash\{\star\}$. The morphisms of $\Sigma_{\mathcal{Q S}}(X)$ are given by the group $\mathcal{U}(2)^{\otimes N}$ of unitary transformations acting by $U_{x} \rho^{(x)} U_{x}^{*}$ on

$$
\rho^{(x)}=\left(\begin{array}{cc}
\alpha_{x} & \theta_{x} \\
\bar{\theta}_{x} & 1-\alpha_{x}
\end{array}\right)
$$

and by collections of isomorphisms of pointed sets.

Proof. Summing functors $\Theta \in \Sigma_{\mathcal{Q} \mathcal{S}_{*}}(X)$ have the properties that $\Theta(\{\star\}, \star)=(\{\star\}, \star)$ the zero object of $\mathcal{Q S}_{*}$ and $\Theta(A \cup B)=\Theta(A) \amalg_{\mathcal{Q S}} \Theta(B)$ for any $A, B \in P(X)$ with $A \cap B=\{\star\}$. We proceed as in the case of Theorem 4.2. The properties of the summing functor implies that it suffices to know the value on sets $\Theta(\{a, \star\})$, since we then obtain

$$
\Theta(A)=\amalg_{a \in A \backslash\{\star\}} \Theta(\{a, \star\})
$$

with the coproduct in $\mathcal{Q S}_{*}$. As in the case of classical probabilities, we consider $\Theta(\{a, \star\})$ as a superposition of the two possible choices of base point $a, \star$ in the set $\{a, \star\}$, except that now, in addition to the superposition we also need to account for 
interference effects. Thus, we assign to $\{a, \star\}$ the object in $\mathcal{Q} \mathcal{S}_{*}$ given by the following set

$$
\Theta(\{a, \star\})= \begin{cases}((\{a, \star\}, \star),(\{a, \star\}, \star)) & \rho_{11}=\alpha_{a} \\ ((\{a, \star\}, \star),(\{a, \star\}, a)) & \rho_{12}=\theta_{a} \\ ((\{a, \star\}, a),(\{a, \star\}, \star)) & \rho_{21}=\bar{\theta}_{a} \\ ((\{a, \star\}, a),(\{a, \star\}, a)) & \rho_{22}=1-\alpha_{a}\end{cases}
$$

This reduces to the classical choice in the diagonal case with $\theta_{a}=0$. This then determines the value $\Theta(A)$ for all $A \in P(X)$ as the a list of $2^{N_{A}} \times 2^{N_{A}}$ pairs of sets of cardinality \#A with associated density matrix $\rho_{A}=\otimes_{a \in A \backslash\{\star\}} \rho^{(a)}$, the $N_{A}$-fold tensor product of the matrices as above

$$
\rho^{(a)}=\left(\begin{array}{cc}
\alpha_{a} & \theta_{a} \\
\bar{\theta}_{a} & 1-\alpha_{a}
\end{array}\right) .
$$

The entries of $\rho_{A}$ can then be identified with the sequences $\left(t_{1}, \ldots, t_{N_{A}}\right)$ with $t_{a} \in$ $\left\{\alpha_{a}, 1-\alpha_{a}, \theta_{a}, \bar{\theta}_{a}\right\}$ for $a \in A \backslash\{\star\}$, where the diagonal entries correspond to those sequences that contain only the letters $\left\{\alpha_{a}, 1-\alpha_{a}\right\}$ as in the classical case. Thus, in order to specify a summing functor $\Theta$ it suffices to assign a choice of values $\left\{\alpha_{x}\right\}_{x \in X \backslash\{\star\}}$ and of $\left\{\theta_{a}\right\}_{x \in X \backslash\{\star\}}$. The only constraint on the choice of the $\alpha_{x}$ comes from the normalization of the trace $\operatorname{Tr}(\rho)=1$, for which, as in the classical case, it suffices to require that all the $\alpha_{x} \in[0,1]$, hence $\left\{\alpha_{x}\right\}_{x \in X \backslash\{\star\}} \in\left|\mathcal{I}^{N}\right|$ with $N=\# X-1$. The constraints on the $\theta_{x}$ come from the requirement that the density matrices satisfy $\rho_{A} \geq 0$. It suffices the ensure that the density matrices $\rho^{(a)}$ of $(9.4)$ have non-negative eigenvalues. The characteristic polynomial $p(\lambda)=\lambda^{2}-\operatorname{Tr}(\rho) \lambda+\operatorname{det}(\rho)=\lambda^{2}-\lambda+\operatorname{det}(\rho)$ has non-negative eigenvalues when the discriminant $\operatorname{Tr}(\rho)^{2}-4 \operatorname{det}(\rho)=1-4 \operatorname{det}(\rho) \geq 0$ and $\operatorname{det}(\rho) \geq 0$. This gives the condition $(9.1)$.

The morphisms in $\Sigma_{\mathcal{Q S}}(X)$ consist of natural transformations of the functors that are isomorphisms on objects. This means isomorphisms $\eta_{A}: \Theta(A) \rightarrow \Theta^{\prime}(A)$ in $\mathcal{Q S}_{*}$ that are compatible with the inclusions $j: A \hookrightarrow A^{\prime}$, with $\eta_{A} \circ \Theta(j)=\Theta^{\prime}(j) \circ$ $\eta_{A}$. An isomorphism $\eta: \rho_{A} X_{A} \rightarrow \rho_{A}^{\prime} X_{A}^{\prime}$ in $\mathcal{Q} \mathcal{S}_{*}$ consists of an invertible quantum channel mapping $\rho_{A}$ to $\rho_{A}^{\prime}$ and a collection of isomorophisms of the pairs of pointed sets in the collections $X_{A}$ and $X_{A}^{\prime}$. The invertible quantum channel is given by a unitary transformation, and the requirement that the isomorphisms are natural transformations of the functors, that is, that they are compatible with the inclusions of subsets, implies that the unitary transformation $\rho_{A}^{\prime}=U_{A} \rho_{A} U_{A}^{*}$ with $U_{A} \in \mathcal{U}\left(2^{N_{A}}\right)$ is a product of unitary transformations of the matrices $\rho^{(a)}$ of (9.4), $U_{A}=U_{a_{1}} \otimes \cdots \otimes$ $U_{a_{N_{A}}}$ with unitaries $U_{a} \in \mathcal{U}(2)$. Note that the relation (9.1) between the off diagonal entry $\theta_{a}$ and the diagonal $\alpha_{a}$ is preserved under the action of $\mathcal{U}(2)$.

Proposition 9.2. The cubical nerve $K=\mathcal{N}_{\mathfrak{C}}\left(\Sigma_{\mathcal{Q S}}(X)\right)$ with $K_{n}=\operatorname{Fun}\left(\mathcal{I}^{n}, \Sigma_{\mathcal{Q S}}(X)\right)$ is given by the action groupoid of $\mathcal{U}(2)^{\otimes N}$ acting on the cubical set

$$
\mathcal{Z}_{N}=\bigcup_{Z \in\left|\mathcal{I}^{N}\right|} \bigcup_{k=0}^{N} \mathcal{I}_{Z}^{k} \times \mathcal{A}_{k}
$$


with $\mathcal{A}_{k}$ a product of $N-k$ annuli (or disks) $\mathcal{A}_{x}$ as in (9.1).

Proof. A functor $\mathcal{I}^{n} \rightarrow \Sigma_{\mathcal{Q S}}(X)$ assigns to each vertex $v \in \mathcal{I}^{n}$ a summing functor $\Theta_{v} \in \Sigma_{\mathcal{Q S}}(X)$, hence a choice of $\left\{\lambda_{x}, \theta_{x}\right\}_{x \in X \backslash\{\star\}}$ satisfying (9.1). Edges of $\mathcal{I}^{n}$ correspond to natural transformations between $\Theta_{v}$ and $\Theta_{v^{\prime}}$ for $\partial(e)=\left\{v, v^{\prime}\right\}$. Let $\mathcal{A}_{\alpha}$ denote as in (9.1) the annulus $\mathcal{A}_{\alpha}=\left\{\alpha(1-\alpha)-1 / 4 \leq|z|^{2} \leq \alpha(1-\alpha)\right\}$, when $\alpha(1-\alpha)>1 / 4$. In the case where $\alpha(1-\alpha) \leq 1 / 4$ we just have the disk $\mathcal{A}_{\alpha}=\left\{|z|^{2} \leq \alpha(1-\alpha)\right\}$. Then the datum of a functor $\mathcal{I}^{n} \rightarrow \Sigma_{\mathcal{Q S}}(X)$ corresponds to assigning for each vertex $v \in \mathcal{I}^{n}$ a union $\cup_{k=0}^{n} \mathbb{P}_{k}(v) \times \mathcal{A}_{k}(v)$ of products of pointed polytopes with $2^{k}$ vertices and products of annuli (or disks) $\mathcal{A}_{k}(v)$, where for a given choice of $\left\{\lambda_{x}, \theta_{x}\right\}_{x \in X \backslash\{\star\}}$ satisfying (9.1), $\mathbb{P}_{k}(v)$ is the polytope given as in 4.13) by the classical probability space $\{0,1\}^{k}$ with probability $\left(t_{1} \ldots t_{k}\right)$ with $t_{i} \in\left\{\alpha_{i}\left(1-\alpha_{i}\right)\right\}$ and $\mathcal{A}_{k}(v)$ is a union of products of annuli $\prod \mathcal{A}_{\alpha_{i}}$, considered with their cubical structure. Arguing as in Proposition 4.3, the functors associated to adjacent vertices, hence the corresponding sequences $t_{1}, \ldots, t_{n}$ in the alphabet $t_{i} \in\left\{\lambda_{x_{i}}^{v}, 1-\alpha_{x_{i}}^{v} \theta_{x_{i}}^{v}, \bar{\theta}_{x_{i}}^{v}\right\}$, are related by a morphism in $\Sigma_{\mathcal{Q} \mathcal{S}_{*}}(X)$. These are unitary transformations in $\mathcal{U}(2)^{\otimes n}$. In particular, since the sequences $s_{1} \ldots s_{n} \in\{0,1\}^{n}$ labeling adjacent vertices of $\mathcal{I}^{n}$ differ at a single digit $s_{k}$, the corresponding sequences $t_{1}, \ldots, t_{n}$ differ in the action of a single $U_{k} \in \mathcal{U}(2)$ relating the density matrices $\rho_{v}^{\left(x_{k}\right)}$ and $\rho_{v^{\prime}}^{\left(x_{k}\right)}$. As in Proposition 4.3 . this reduces the choices of the data $\left\{\alpha_{x}^{v}, \theta_{x}^{v}\right\}$ to a single choice $\left\{\alpha_{x}, \theta_{x}\right\}_{x \in X \backslash\{\star\}}$ at a single vertex, with the assignments at a the other vertices of the cube obtained by applying unitary transformations associated to the edges of the cube. Thus, the cubical nerve $\mathcal{N}_{\mathfrak{C}}\left(\Sigma_{\mathcal{Q S}}(X)\right)$ can be described as the action groupoid of the action of $\mathcal{U}^{\otimes N}$ on the set $\mathcal{Z}_{N}$ of $(9.5)$, which parameterizes the choice of data $\left\{\alpha_{x}, \theta_{x}\right\}_{x \in X \backslash\{\star\}}$.

Lemma 9.3. The simplicial set $F_{\mathcal{Q S}}(X)=\mathcal{N}_{\mathfrak{C}}\left(\Sigma_{\mathcal{Q S}}(X)\right)$ is homotopy equivalent to the Borel homotopy quotient $\mathcal{M}_{G}=E G \times_{G} \mathcal{Z}_{N}$, with $G=\mathcal{U}(2)^{\otimes N}$, for $N=\# X-1$, and $\mathcal{Z}_{N}$ the set $(9.5$.

Proof. Proposition 9.2 shows that the category $\Sigma_{\mathcal{Q S}}(X)$ of summing functors can be identified with the action groupoid of the group of unitary transformations $\mathcal{U}(2)^{\otimes N}$ acting on density matrices of the form $\rho=\otimes_{x} \rho^{(x)}$, or equivalently on the set $\mathcal{Z}_{N}$ of (9.5) that parameterizes them. Thus, the nerve $\mathcal{N} \Sigma_{\mathcal{Q} \mathcal{S}_{*}}(X)$ can be identified with the classifying space $B \mathcal{G}$ of the action groupoid $\mathcal{G}=\mathcal{Z}_{N} \rtimes \mathcal{U}(2)^{\otimes N}$, with $N=\# X-1$. The classifying space $B \mathcal{G}$ of an action groupoid $\mathcal{G}=\mathcal{Z} \rtimes G$ of a Lie group action on a manifold is homotopy equivalent to the Borel construction of the homotopy quotient $\mathcal{Z}_{G}=E G \times_{G} \mathcal{Z}$ (see e.g. Proposition B.11 of [35]).

Corollary 9.4. The geometric realization $\left|\mathcal{N}_{\mathfrak{C}}\left(\Sigma_{\mathcal{Q S}}(X)\right)\right|$ is homotopy equivalent to a union of strata of the form

$$
\mathcal{Z}_{N, \mathcal{P}}^{j} \times B\left(\mathcal{U}(2)^{\otimes j} \otimes(\mathcal{U}(1) \times \mathcal{U}(1))^{\otimes(N-j)}\right)
$$


where $\mathcal{Z}_{N, \mathcal{P}}^{j} \subset\left|\mathcal{N}_{\mathcal{C}} \Sigma_{\mathcal{P S}}(X)\right|$ is a subset of the realization of $F_{\mathcal{P} \mathcal{S}_{*}}(X)$, the value of the $\Gamma$-space of classical probabilities, given by

$$
\mathcal{Z}_{N, \mathcal{P}}^{j}=\bigcup_{Z \in \mathcal{S}_{j}}\left|\mathcal{I}_{Z}^{N}\right|
$$

with $\mathcal{S}_{j}$ the set of sequences $\left\{\alpha_{x}\right\}$ with $j$ entries equal to $1 / 2$, and where $B\left(\mathcal{U}(2)^{\otimes j} \otimes\right.$ $\left.(\mathcal{U}(1) \times \mathcal{U}(1))^{\otimes(N-j)}\right)$ are the classifying spaces of the stabilizers $\mathcal{U}(2)^{\otimes j} \otimes(\mathcal{U}(1) \times$ $\mathcal{U}(1))^{\otimes(N-j)}$ of the strata $\mathcal{Z}_{N}^{j}$ of $\mathcal{Z}_{N}$ with $\mathcal{Z}_{N, \mathcal{P}}^{j}=\mathcal{Z}_{N}^{j} / \mathcal{U}(2)^{\otimes N}$.

Proof. The homotopy quotient $\mathcal{Z}_{G}$ has projection maps

$$
B G \stackrel{\pi_{1}}{\longleftarrow} \mathcal{Z}_{G} \stackrel{\pi_{2}}{\longrightarrow} \mathcal{Z} / G
$$

where the projection $\pi_{1}$ is a fibration over $B G$ with fiber $\mathcal{Z}$, while the preimage of a point $x \in \mathcal{Z} / G$ is a copy of $B G_{x}$ with $G_{x} \subset G$ the isotropy group. In the case of the set $\mathcal{Z}_{N}$ of $(9.5)$ with the action of $G=\mathcal{U}(2)^{\otimes N}$, we can decompose $\mathcal{Z}_{N}$ into strata with associated stabilizers of the action. The top stratum $\mathcal{Z}_{N}^{0}$ consists of $\mathcal{Z}_{N}^{0}=$ $\cup_{Z \in \mathcal{S}_{0} \subset\left|\mathcal{I}^{N}\right|} \cup_{k} \mathcal{I}_{Z}^{k} \times \mathcal{A}_{k}$, where $\mathcal{S}_{0}$ is the set of those sequences $Z=\left\{\alpha_{x}\right\}_{x \in X \backslash\{\star\}}$ where none of the $\alpha_{x}$ is equal to $1 / 2$. The lower strata $\mathcal{Z}_{N}^{j}$ consist of $\mathcal{Z}_{N}^{0}=\cup_{Z \in \mathcal{S}_{j} \subset\left|\mathcal{I}^{N}\right|} \cup_{k}$ $\mathcal{I}_{Z}^{k} \times \mathcal{A}_{k}$ where $\mathcal{S}_{j}$ is the set of sequences $Z=\left\{\alpha_{x}\right\}$ where $j$ of the $\alpha_{x}$ are equal to $1 / 2$. Since in the $\mathcal{U}(2)$ action $\rho^{(x)} \mapsto U_{x} \rho^{(x)} U_{x}^{*}$ we can identify unitaries $U_{x} \in \mathcal{U}(2)$ up to phase factors (diagonal unitaries) $\mathcal{U}(1) \times \mathcal{U}(1)$, the stabilizer of the top stratum is $(\mathcal{U}(1) \times \mathcal{U}(1))^{\otimes N}$. The stratum $\mathcal{Z}_{N}^{j}$ has stabilizer $\mathcal{U}(2)^{\otimes j} \otimes(\mathcal{U}(1) \times \mathcal{U}(1))^{\otimes(N-j)}$. The quotient $\mathcal{Z}_{N} / \mathcal{U}(2)^{\otimes N}$ is correspondingly decomposed into strata, where up to the action of $\mathcal{U}(2)$ we can identify the density matrices $\rho^{(x)}$ with diagonal classical probabilities. This implies that we can identify the quotient $\mathcal{Z}_{N} / \mathcal{U}(2)^{\otimes N}$ with a classical space

$$
\left|\mathcal{N}_{\mathcal{C}} \Sigma_{\mathcal{P S}}(X)\right|=\bigcup_{Z \in\left|\mathcal{I}^{N}\right|}\left|\mathcal{I}_{Z}^{N}\right|
$$

with a stratification by 9.7 . Over each set $\mathcal{Z}_{N, \mathcal{P}}^{j}$ we have a copy of the fiber $B\left(\mathcal{U}(2)^{\otimes j} \otimes(\mathcal{U}(1) \times \mathcal{U}(1))^{\otimes(N-j)}\right.$.

Remark 9.5. The geometric realization of the nerve $\mathcal{N}_{\mathfrak{C}}\left(\Sigma_{\mathcal{Q} \mathcal{S}_{*}}(X)\right)$ is more interesting topologically than the case of classical probabilities, due to the presence of the classifying spaces of unitary groups.

Remark 9.6. We obtain a $\Gamma$-space of quantum pointed sets $F_{\mathcal{Q S}}: \Gamma^{0} \rightarrow \square_{*}$ that assigns to a pointed set $(X, \star)$ the cubical nerve $\mathcal{N}_{\mathfrak{C}}\left(\Sigma_{\mathcal{Q S}}(X)\right)$ of the category of summing functors of Theorem 9.1. This can be extended to an endofunctor $F_{\mathcal{Q S}}$ : $\square_{*} \rightarrow \square_{*}$ and determines an associated homotopy theoretic spectrum, by the Segal construction [30]. 
9.2. The stochastic Gamma spaces. As in the case of classical probabilities, we can associate to a category $\mathcal{Q C}$ a probabilistic $\Gamma$-space $F_{\mathcal{Q C}}: \mathcal{P} \mathcal{S}_{*} \rightarrow \mathcal{P} \square_{*}$. The construction is analogous to the case of $F_{\mathcal{P C}}: \mathcal{P} \mathcal{S}_{*} \rightarrow \mathcal{P} \square_{*}$ that we discussed earlier. By Lemma 5.7 the functor $F_{\mathcal{Q C}}$ maps a probabilistic pointed set $\Lambda X$ to the cubical nerve $\mathcal{N}_{\mathfrak{C}}\left(\mathcal{P} \Sigma_{\mathcal{Q C}}(\Lambda X)\right)$, which can be identified with the probabilistic pointed cubical set $\sum_{i} \lambda_{i} \mathcal{N}_{\mathfrak{C}}\left(\Sigma_{\mathcal{P Q}}\left(X_{i}, x_{i}\right)\right)$.

\section{Gamma SPACEs, SPECTRA, AND GAPPED Systems}

There has been a growing interest recently in the use of homotopy theoretic methods in the modeling of topological phases of matter. In particular, the use of spectra to classify symmetry protected topological phases in terms of generalized cohomology groups associated to a spectrum of invertible physical systems, [21]. This idea is elaborated upon in [15], where a spectrum $\mathrm{GP}_{n}^{\times}$of invertible gapped phases of matter is considered, with homotopy equivalences $\Omega \mathrm{GP}_{n}^{\times} \rightarrow \mathrm{GP}_{n-1}^{\times}$corresponding to realizing a continuous transition between $n$-dimensional systems in the same phase via an invertible $(n-1)$-dimensional interface (invertible defect).

We consider here a similar idea, from the point of view of Segal's $\Gamma$-spaces and we present a construction of $\Gamma$-spaces associated to gapped systems. Our setting here is only a simplified model of the properties one usually requires for gapped systems, see $\S 5.2$ of [38]. In general, in addition to the existence of a gap $\Delta>0$ in the spectrum, one also requires a uniform bound on the degeneracy of the ground state, namely the condition that for all $\left(X, H_{X}\right)$ the ground state degeneracy satisfies $1 \leq \operatorname{dim} \operatorname{Ker}\left(H_{X}\right) \leq m$ with some uniform bound by some fixed $m \in \mathbb{N}$. In the setting we consider here, this condition would not be compatible with the categorical sum. In order to obtain a more sophisticated model for gapped systems where the uniformly bounded degeneracy condition can also be imposed, we need to work with a different categorical setting. This will be investigated elsewhere.

10.1. Gamma spaces of gapped systems. In this setting, instead of considering the category $\mathcal{F} \mathcal{Q}$ of finite quantum probabilities (density matrices) with morphisms given by quantum channels, one considers a category $\mathcal{F} \mathcal{Q}^{\Delta}$ where the objects are systems $\left(X, H_{X}\right)$ with a Hamiltonian $H_{X}$ acting on a HIlbert space $\mathcal{H}_{X}=\oplus_{x \in X} \mathcal{V}_{x}$ with a fixed internal space $\mathcal{V}_{x}$ (which for simplicity we will just take equal to a line $\mathbb{C}_{x}$ ), with the property that $H_{X}^{*}=H_{X}$ and that $H_{X}$ has a gap in the spectrum above the ground level, that is, $0 \in \operatorname{Spec}\left(H_{X}\right)$ and $\operatorname{Spec}\left(H_{X}\right) \subset\{0\} \cup[\Delta, \infty)$. We realize objects in $\mathcal{F} \mathcal{Q}^{\Delta}$ as objects of $\mathcal{F} \mathcal{Q}$ by associating to a pair $\left(X, H_{X}\right)$ the pair $\left(X, \rho_{X}\right)$ in $\mathcal{F} \mathcal{Q}$ with

$$
\rho_{X}=\frac{e^{-\beta H_{X}}}{\operatorname{Tr}\left(e^{-\beta H_{X}}\right)},
$$

where $\beta>0$ is a fixed inverse temperature parameter. Using this identification of objects of $\mathcal{F} \mathcal{Q}^{\Delta}$ with a subset of objects of $\mathcal{F} \mathcal{Q}$, we take the morphisms in $\mathcal{F} \mathcal{Q}^{\Delta}$ to be induced by the morphisms in $\mathcal{F} \mathcal{Q}$. Namely, morphisms in $\operatorname{Mor}_{\mathcal{F} \mathcal{Q}^{\Delta}}\left(\left(X, H_{X}\right),\left(Y, H_{Y}\right)\right)$ are gap preserving quantum channels, $\Phi\left(\rho_{X}\right)=\rho_{Y}$. 
When we apply the previous construction of quantum categories $\mathcal{Q C}$ using the category $\mathcal{F} \mathcal{Q}^{\Delta}$ instead of $\mathcal{F} \mathcal{Q}$, we obtain a subcategory $\mathcal{Q} \mathcal{C}^{\Delta}$ of $\mathcal{Q} \mathcal{C}$, which is described as follows.

Definition 10.1. The gapped quantum category $\mathcal{Q C}^{\Delta}$ has objects that include the zero object of $\mathcal{Q C}$ and objects $\rho C=\left(\left(C_{i}, C_{j}\right), \rho_{i j}\right)$ of $\mathcal{Q C}$ where the density matrix $\rho$ is of the form

$$
\rho=\frac{e^{-\beta H}}{\operatorname{Tr}\left(e^{-\beta H}\right)},
$$

for some (fixed) inverse temperature parameter $\beta>0$, with the property that the Hamiltonian $H$ has spectrum $\operatorname{Spec}(H) \subset\{0\} \cup[\Delta, \infty)$, for a fixed gap $\Delta>0$. The morphisms in $\mathcal{Q C}^{\Delta}$ are induced by the morphisms in $\mathcal{Q C}$.

Lemma 10.2. The coproduct in $\mathcal{Q C}$ induces a coproduct in $\mathcal{Q C}^{\Delta}$.

Proof. The coproduct $\rho C \amalg_{\mathcal{Q C}} \rho^{\prime} C^{\prime}$ of two objects $\rho C, \rho^{\prime} C^{\prime} \in \mathcal{Q C}^{\Delta}$ has density matrix given by the product $\rho \otimes \rho^{\prime}$. We have $\rho=e^{-\beta H} / \operatorname{Tr}\left(e^{-\beta H}\right)$ and $\rho^{\prime}=e^{-\beta H^{\prime}} / \operatorname{Tr}\left(e^{-\beta H^{\prime}}\right)$ where the respective Hamiltonians $H, H^{\prime}$ have spectrum contained in $\{0\} \cup[\Delta, \infty)$. The tensor product $\rho \otimes \rho^{\prime}$ corresponds to the Kronecker sum $H \oplus H^{\prime}=H \otimes 1+1 \otimes H^{\prime}$ of the Hamiltonians. The spectrum of the Kronecker sum $\operatorname{Spec}\left(H \otimes 1+1 \otimes H^{\prime}\right)$ is given by sums of eigenvalues of $H$ and $H^{\prime}$, hence it is still contained in $\{0\} \cup[\Delta, \infty)$, so that the coproduct in $\mathcal{Q C}$ of two objects in $\mathcal{Q C}^{\Delta}$ is still an object in $\mathcal{Q} \mathcal{C}^{\Delta}$.

We focus on the case where $\mathcal{C}=\mathcal{S}_{*}$, the category of finite pointed sets. As in the case of $\mathcal{Q S}_{*}$, we construct the associated $\Gamma$-space by constructing the category $\Sigma_{\mathcal{Q S}_{*}^{\Delta}}(X)$ of summing functors $\Theta: P(X) \rightarrow \mathcal{Q S}_{*}^{\Delta}$.

Proposition 10.3. For sufficiently large $\beta>0$, an object $\Theta$ in $\Sigma_{\mathcal{Q S}_{*}}(X)$ is specified by the choice of a point $\alpha=\left\{\alpha_{x}\right\}_{x \in X \backslash\{\star\}} \in \mathcal{I}_{\beta, \Delta}^{N}$ for an interval $\mathcal{I}_{\beta, \Delta}=\left[a_{\beta, \Delta}, b_{\beta, \Delta}\right] \subset$ $[0,1]$ and with $N=\# X-1$, and a choice of $\left\{\theta_{x}\right\}_{x \in X \backslash\{\star\}} \in T_{r(Z)}^{N}$, where $T^{N}=$ $\left(S^{1}\right)^{N}$ is a torus and the subscript $r(Z)$ indicates that the $k$-th circle has a radius $r=r\left(\alpha_{x}, \beta, \Delta\right)$ uniquely determined by the choice of $\alpha_{x}$ and by the fixed values of $\Delta$ and $\beta$. The morphisms in $\Sigma_{\mathcal{Q S}_{*}}(X)$ are given by unitary transformations in $\mathcal{U}(2)^{\otimes N}$ and by collections of isomorphisms of pointed sets.

Proof. As in the case of $\mathcal{Q S}_{*}^{\Delta}$, we know that the values $\Theta(A)$ are given by coproducts (9.2) in $\mathcal{Q S}_{*}^{\Delta}$, with the terms $\Theta(\{a, \star\})$ as in (9.3). In this case, the density matrix (9.4) associated to $\Theta(\{a, \star\})$ will have to satisfy additional constraints due to the gap condition on the spectrum of the associated Hamiltonian. In the case of a $2 \times 2$ matrix, the condition that the spectrum has a gap of width $\Delta$ above the ground level $\lambda=0$ corresponds to requiring that one of the eigenvalues is zero and the other one is equal to the width of the gap $\Delta$. This means that the spectrum of the corresponding density matrix $\rho=e^{-\beta H} / \operatorname{Tr}\left(e^{-\beta H}\right)$ is given by

$$
\operatorname{Spec}(\rho)=\left\{\frac{e^{-\beta \Delta}}{1+e^{-\beta \Delta}}, \frac{1}{1+e^{-\beta \Delta}}\right\} .
$$


Let $q=1-\alpha(1-\alpha)+|\theta|^{2}$. Then the condition above on the eigenvalues of $(9.4)$ gives

which gives

$$
\frac{1}{2}\left(1-q^{1 / 2}\right)=\frac{e^{-\beta \Delta}}{1+e^{-\beta \Delta}}, \quad \frac{1}{2}\left(1+q^{1 / 2}\right)=\frac{1}{1+e^{-\beta \Delta}},
$$

This then gives the relation

$$
q^{1 / 2}=\frac{1-e^{-\beta \Delta}}{1+e^{-\beta \Delta}}
$$

$$
|\theta|^{2}=\left(\frac{1-e^{-\beta \Delta}}{1+e^{-\beta \Delta}}\right)^{2}-1+\alpha(1-\alpha)=\frac{-4 e^{-\beta \Delta}}{\left(1+e^{-\beta \Delta}\right)^{2}}+\alpha(1-\alpha),
$$

where $0<4 e^{-\beta \Delta} /\left(1+e^{-\beta \Delta}\right)^{2} \leq 1$. There is an interval of values $0<e^{-\beta \Delta} \leq u_{\beta, \Delta}$ with $u_{\beta, \Delta}<1$ such that the discriminant of $\frac{-4 e^{-\beta \Delta}}{\left(1+e^{-\beta \Delta}\right)^{2}}+\alpha(1-\alpha)=0$, seen as an equation in $\alpha$, is non-negative. Then the right-hand-side of $(10.2)$ is non-negative for $\alpha$ in the interval $\left[a_{\beta, \Delta}, b_{\beta, \Delta}\right]$ between the two roots. For a fixed value of the gap $\Delta$, it is always possible to choose an inverse temperature $\beta>0$ sufficiently large so that the condition $e^{-\beta \Delta} \leq u_{\beta, \Delta}$ is satisfied. For such a choice of $\beta$, one then obtains solutions of $(10.2)$ given by any choice of $\alpha \in\left[a_{\beta, \Delta}, b_{\beta, \Delta}\right]$ and a circle of values of $\theta$ with radius $r=r(\alpha, \Delta, \beta)$ fixed by the relation 110.2$)$ (and depending on $\alpha$ and on $\Delta$ and $\beta$ ). The morphisms in in $\Sigma_{\mathcal{Q S}_{*}^{*}}(X)$ are given by unitary transformations in $\mathcal{U}(2)^{\otimes N}$ acting by $U_{x} \rho^{(x)} U_{x}^{*}$ on the density matrices $\rho^{(x)}$ as in Theorem 9.1, and by collections of isomorphisms between the pointed sets in the two objects. Since the locus determined by the relation 10.2 is specified by the condition on the spectrum of the matrices $\rho^{(x)}$, it is preserved by unitary transformations.

Proposition 10.4. The nerve $\mathcal{N}_{\mathfrak{C}}\left(\Sigma_{\mathcal{Q S}_{*}}(X)\right)$ is the action groupoid of the $\mathcal{U}(2)^{\otimes N}$ action on the cubical set

$$
\mathcal{Z}_{N, \Delta}=\bigcup_{Z \in\left|\mathcal{I}_{\beta, \Delta}^{N}\right|} \bigcup_{k=0}^{N} \mathcal{I}_{Z}^{k} \times T_{r(Z)}^{N-k}
$$

The geometric realization $\left|\mathcal{N}_{\mathfrak{C}}\left(\Sigma_{\mathcal{Q S}_{*}^{\Delta}}(X)\right)\right|$ is homotopy equivalent to a union of strata of the form (9.6) where the $\mathcal{S}_{j} \subset\left|\mathcal{I}_{\beta, \Delta}^{N}\right|$ consists of all the sequences $\left\{\alpha_{x}\right\} \in\left[a_{\beta, \Delta}, b_{\beta, \Delta}\right]^{N}$ where $j$ of the terms are equal to $1 / 2$.

Proof. The argument is analogous to Proposition 9.2 and Corollary 9.4. The nerve $\mathcal{N}_{\mathfrak{C}}\left(\Sigma_{\mathcal{Q} \mathcal{S}_{*}}(X)\right)$ is constructed as in the case of $\mathcal{Q} \mathcal{S}_{*}$, except that in this case the annuli and disks are replaced by circles $\theta_{x} \in S_{r(\alpha, \Delta, \beta)}^{1}$ of radius determined by 10.2). We write $T_{r(Z)}^{N-k}$ for the product of these $N-k$ circles, where we write $r(Z)$ for this dependence of the radii on the $\alpha_{x}$, leaving the dependence on $\beta$ and $\Delta$ implicit. For fixed $\Delta$, we are choosing $\beta>0$ large enough as in Proposition 10.3 , so that for $\alpha$ in the subinterval $\left[a_{\beta, \Delta}, b_{\beta, \Delta}\right] \subset[0,1]$ the estimate $\frac{-4 e^{-\beta \Delta}}{\left(1+e^{-\beta \Delta}\right)^{2}}+\alpha(1-\alpha) \geq 0$ holds. The interval $\left[a_{\beta, \Delta}, b_{\beta, \Delta}\right]$ contains the point $\alpha=1 / 2$ as one can verify directly. 
Remark 10.5. The $\Gamma$-space $F_{\mathcal{Q S}_{*}^{\star}}: \Gamma^{0} \rightarrow \square_{*}$ obtained in this way can be extended to an endofunctor $F_{\mathcal{Q S}_{*}^{*}}: \square_{*} \rightarrow \square_{*}$ and determined an associated connective spectrum by the Segal construction of [30]. This provides then a construction of a homotopy theoretic spectrum associated to a category of gapped systems with a fixed gap $\Delta$.

Remark 10.6. As in the cases of $\mathcal{P} \mathcal{S}_{*}$ and of $\mathcal{Q} \mathcal{S}_{*}$ it is also possible to extend the $\Gamma$-space $F_{\mathcal{Q S}_{*}^{\Delta}}: \Gamma^{0} \rightarrow \square_{*}$ to a probabilistic $\Gamma$-space $F_{\mathcal{Q S}}: \mathcal{P} \mathcal{S}_{*} \rightarrow \mathcal{P} \square_{*}$.

10.2. Gamma spaces and gapped phases. We consider here a different construction of a $\Gamma$-space related to gapped system, where instead of fixing the gap and restricting the category $\mathcal{F} \mathcal{Q}$ to a subcategory $\mathcal{F} \mathcal{Q}^{\Delta}$ of gapped systems and gap preserving quantum channels, we consider all the objects of $\mathcal{F} \mathcal{Q}$, so that there is no fixed gap, but we change the morphisms so that we regard all the quantum channels that preserve a gap $\Delta>0$ as isomorphisms. We can do this in the form of a localization of the category $\mathcal{F} \mathcal{Q}$ at a collection of morphisms $\mathcal{T}_{\Delta}$.

More precisely, the set of morphisms $\mathcal{T}_{\Delta}$ consists of all morphisms in $\mathcal{Q S}_{*}$ where both source and target are objects in $\mathcal{Q S}_{*}^{\Delta}$. The localization $\mathcal{Q} \mathcal{S}_{*}\left[\mathcal{T}_{\Delta}^{-1}\right]$ is obtained as a quotient of the path category $\mathcal{P}\left(\mathcal{Q S}_{*}, \mathcal{T}_{\Delta}^{-1}\right)$. The path category has the same objects as $\mathcal{Q S}_{*}$ and morphisms given by arbitrary concatenations $\Psi_{1} \cdots \Psi_{N}$ where the $\Psi_{i}$ are either morphisms in $\mathcal{Q S}_{*}$ or formal inverses of morphisms in $\mathcal{T}_{\Delta}$, with the target of $\Psi_{i}$ equal to the source of $\Psi_{i+1}$. The equivalence relation on $\mathcal{P}\left(\mathcal{Q S}_{*}, \mathcal{T}_{\Delta}^{-1}\right)$ identifies the empty string at a given object with the identity morphism, a string $\Psi_{1} \Psi_{2}$ where both $\Psi_{i}$ are morphisms in $\mathcal{Q S}_{*}$ with the morphism $\Psi_{2} \circ \Psi_{1}$ and a string $\Phi^{-1} \Phi$ or $\Phi \Phi^{-1}$, with $\Phi \in \mathcal{T}_{\Delta}$ and $\Phi^{-1}$ its formal inverse, with the identity morphism on the source, respectively target, of $\Phi$. This determines a category

$$
\mathcal{Q S}_{*}\left[\mathcal{T}_{\Delta}^{-1}\right]=\mathcal{P}\left(\mathcal{Q S} \mathcal{S}_{*}, \mathcal{T}_{\Delta}^{-1}\right) / \sim
$$

with a localization functor $\mathcal{Q S}_{*} \rightarrow \mathcal{Q} \mathcal{S}_{*}\left[\mathcal{T}_{\Delta}^{-1}\right]$ which maps morphisms in $\mathcal{S}_{\Delta}$ to isomorphisms. We refer the reader to the overview in $\S 2$ of [14].

The category $\mathcal{Q S}_{*}\left[\mathcal{T}_{\Delta}^{-1}\right]$ has zero object and categorical sum inherited from $\mathcal{Q S}_{*}$. Thus, we can consider the $\Gamma$-space $F_{\mathcal{Q S}}\left[\mathcal{T}_{\Delta}^{-1}\right]: \Gamma^{0} \rightarrow \square_{*}$ associated to $\mathcal{Q} \mathcal{S}_{*}\left[\mathcal{T}_{\Delta}^{-1}\right]$.

Proposition 10.7. The summing functors $\Theta: P(X) \rightarrow \mathcal{Q S}_{*}\left[\mathcal{T}_{\Delta}^{-1}\right]$ are specifed by data $\left\{\alpha_{x}\right\}_{x \in X \backslash\{\star\}} \in\left|\mathcal{I}^{N}\right|$ and $\left\{\theta_{x}\right\} \in \mathcal{A}_{x}$ as in the case of $\mathcal{Q S}_{*}$. For sufficiently large $\beta>0$, the morphisms in $\Sigma_{\mathcal{Q} \mathcal{S}_{*}\left[\mathcal{T}_{\Delta}^{-1}\right]}(X)$ are given by unitary transformations in $\mathcal{U}(2)^{\otimes N}$ and by quantum channels $\Phi^{(x)}$ with $\Phi^{(x)} \rho^{(x)}=\rho^{\prime(x)}$ whenever both $\rho^{(x)}$ and $\rho^{\prime(x)}$ have entries satisfying the relation 10.2 .

Proof. The characterization of objects in $\Sigma_{\mathcal{Q} \mathcal{S}_{*}\left[\mathcal{T}_{\Delta}^{-1}\right]}(X)$ is as in the case of the category $\mathcal{Q} \mathcal{S}_{*}$. Morphisms in the category $\Sigma_{\mathcal{Q} \mathcal{S}_{*}\left[\mathcal{T}_{\Delta}^{-1}\right]}(X)$ of summing functors are isomorphisms in $\mathcal{Q} \mathcal{S}_{*}\left[\mathcal{T}_{\Delta}^{-1}\right]$ compatible with the inclusions of subsets in $P(X)$. These isomorphisms are generated by unitary transformations $\mathcal{U}(2)^{\otimes N}$ and by morphisms in $\mathcal{T}_{\Delta}$, and by isomorphisms of pointed sets. A morphism in $\mathcal{T}_{\Delta}$ compatible with the inclusions can 
be expressed in terms of quantum channels relating the density matrices $\rho^{(x)}$ of the source and target object where both source and target are in $\mathcal{Q S}_{*}^{\Delta}$.

Corollary 10.8. The nerve $\mathcal{N}_{\mathfrak{C}}\left(\Sigma_{\mathcal{Q} \mathcal{S}_{*}\left[\mathcal{T}_{\Delta}^{-1}\right]}(X)\right)$ is given by the homotopy quotient of the equivalence relation on the set $\mathcal{Z}_{N}$ of $(9.5)$ generated by quantum channels that preserve the $\Delta$-gap in the spectrum.

Proof. By the same argument used in the case of the category $\mathcal{Q S}_{*}$, we identify the cubical nerve with the groupoid of the equivalence relation on $\mathcal{Z}_{N}$ given by unitary equivalence implemented by the group $\mathcal{U}(2)^{\otimes N}$ together with the action of the gap preserving quantum channels in $\mathcal{T}_{\Delta}$.

Remark 10.9. Equivalent objects in $\mathcal{N}_{\mathfrak{C}}\left(\Sigma_{\mathcal{Q S}_{*}\left[\mathcal{T}_{\Delta}^{-1}\right]}(X)\right)$ under unitaries in $\mathcal{U}(2)^{\otimes N}$ and quantum channels in $\mathcal{T}_{\Delta}$ corresponds to system in the same topological phase, in the sense that they are mapped to one another in such a way that the gap $\Delta$ is preserved.

The spectra associated to the $\Gamma$-spaces $\mathcal{F}_{\mathcal{Q S}_{*}^{*}}: \Gamma^{0} \rightarrow \square_{*}$ and $\mathcal{F}_{\mathcal{Q S}_{*}\left[\mathcal{T}_{\Delta}^{-1}\right]}: \Gamma^{0} \rightarrow \square_{*}$ are obtained by considering the corresponding extensions to endofunctors of $\square_{*}$,

$$
F_{\mathcal{C}}(K)=\int^{n} K_{n} \wedge F_{\mathcal{C}}(\{0, \ldots, n\})
$$

for $\mathcal{C}$ equal to either $\mathcal{Q S}_{*}^{\Delta}$ or $\mathcal{Q} \mathcal{S}_{*}\left[\mathcal{T}_{\Delta}^{-1}\right]$. In the first case, the cubical set $\mathcal{F}_{\mathcal{Q S}_{*}}(K)$, for an $n$-dimensional cubical set $K$, describes the homotopy quotient of the unitary equivalence relation on systems on the $n$-dimensional $K$ with gap $\Delta$, while in the second case it describes the homotopy quotient of the equivalence relation by gap preserving quantum channels on all systems on the $n$-dimensional sets. The structure maps $S^{1} \wedge F_{\mathcal{C}}\left(S^{n}\right) \rightarrow F_{\mathcal{C}}\left(S^{n+1}\right)$ relate these equivalences of systems in different dimensions via a suspension operation.

Acknowledgment. The author is extremely grateful to Tobias Fritz for many useful discussions and for providing many comments and suggestions. She also thanks Paolo Aluffi, Tom Leinster, and Jack Morava for helpful comments. The author is partially supported by NSF grant DMS-1707882, and by NSERC Discovery Grant RGPIN-2018-04937 and Accelerator Supplement grant RGPAS-2018-522593, and by the Perimeter Institute for Theoretical Physics. 


\section{REFERENCES}

[1] R. Antolini, Geometric realisations of cubical sets with connections, and classifying spaces of categories, Appl. Categ. Structures 10 (2002), no. 5, 481-494.

[2] J.C. Baez, T. Fritz, T. Leinster, A characterization of entropy in terms of information loss, Entropy 13 (2011) no. 11, 1945-1957.

[3] J.C. Baez, T. Fritz A Bayesian characterization of relative entropy, Theory Appl. Categ. 29 (2014) No. 16, 422-457.

[4] A.K. Bousfield, E.M. Friedlander, Homotopy theory of $\Gamma$-spaces, spectra, and bisimplicial sets, in "Geometric applications of homotopy theory II", Lecture Notes in Math. 658 (1978) 80-130.

[5] R. Brown, P.J. Higgins, On the algebra of cubes, J. Pure Appl. Algebra 21 (1981) 233-260.

[6] G. Carlsson, Topology and data, Bull. Amer. Math. Soc. (N.S.) 46 (2009), no. 2, 255-308.

[7] C. Curto, What can topology tell us about the neural code? Bull. Amer. Math. Soc. (N.S.) 54 (2017), no. 1, 63-78.

[8] V.A. Efremovich, Yu.B. Rudyak, On the concept of the Euler characteristic, Uspehi Mat. Nauk 31 (1976) 5(191), 239-240

[9] D.K. Faddeev, On the concept of entropy of a finite probabilistic scheme, Uspehi Mat. Nauk (N.S.) 11 (1956), no. 1(67), 227-231.

[10] R. Fenn, C. Rourke, B. Sanderson, Trunks and classifying spaces, Applied Categorical Structures, Vol.3 (1995), N.4, 321-356.

[11] U. Franz, What is stochastic independence? in "Non-commutativity, infinite-dimensionality and probability at the crossroads", pp. 254-274, QP-PQ: Quantum Probab. White Noise Anal., 16, World Scientific, 2002.

[12] G. Friedman, An elementary illustrated introduction to simplicial sets, Rocky Mountain J. Math. 42 (2012), no. 2, 353-423.

[13] T. Fritz, A presentation of the category of stochastic matrices, arXiv:0902.2554

[14] T. Fritz, Categories of Fractions Revisited, arXiv:0803.2587

[15] D. Gaiotto, T. Johnson-Freyd, Symmetry protected topological phases and generalized cohomology, arXiv:1712.07950 [hep-th]

[16] U. Grenander, Probabilities on Algebraic Structures, Dover, 2008.

[17] M. Hovey, Model categories, American Mathematical Society, 2007.

[18] J.F. Jardine, Cubical homotopy theory: a beginning, preprint, 2002. https://www.newton.ac.uk/files/preprints/ni02030.pdf

[19] D.M. Kan, Abstract homotopy. I, Proc. Nat. Acad. Sci. U.S.A. 41 (1955), 1092-1096.

[20] A.I. Khinchin, Mathematical Foundations of Information Theory, Dover, 1957.

[21] A. Kitaev, On the classification of short-range entangled states, talk at Simons Center, 2013, http://scgp.stonybrook.edu/video_portal/video.php?id=2010

[22] T. Leinster, The categorical origins of entropy, lecture at "Topological and Geometric Structures of Information", CIRM, Luminy, 2017.

[23] T. Leinster, Monoidal Categories with Projections, in n-Category Café, August 2016 https://golem.ph.utexas.edu/category/2016/08/monoidal_categories_with_proje.html

[24] N. Levitt, The Euler characteristic is the unique locally determined numerical homotopy invariant of finite complexes, Discrete Comput. Geom. 7 (1992), no. 1, 59-67.

[25] M. Lydakis, Smash products and $\Gamma$-spaces, Math. Proc. Cam. Phil. Soc. 126 (1999) 311-328.

[26] Yu.I. Manin, Neural codes and homotopy types: mathematical models of place field recognition, Mosc. Math. J. 15 (2015) no. 4, 741-748.

[27] M. Marcolli, D. Tsao, Geometry of Neuroscience, book in preparation.

[28] J.P. May, Simplicial Objects in Algebraic Topology, University of Chicago Press, 1967.

[29] S. Schwede, Stable homotopical algebra and $\Gamma$-spaces, Math. Proc. Phil. Soc. Vol.126 (1999) 329-356.

[30] G. Segal, Categories and cohomology theories, Topology, Vol.13 (1974) 293-312. 
[31] R.M. Shortt, Combinatorial methods in the study of marginal problems over separable spacee, J. Math. Analysis and Appl. 97 (1983) 462-479.

[32] J. Strom, Modern Classical Homotopy Theory, American Mathematical Society, 2011.

[33] R.W. Thomason, Symmetric monoidal categories model all connective spectra, Theory and Applications of Categories, Vol.1 (1995) N.5, 78-118.

[34] N.N. Vorobev, Consistent families of measures and their extensions, Theory of Probability and its Applications, VII (1962) N.2, 147-163.

[35] J. Watts, Symplectic quotients and representability: the circle action case, arXiv:1610.01547v2

[36] C.Z. Xiong, Minimalist approach to the classification of symmetry protected topological phases, arXiv: $1701.00004 \mathrm{v} 2$

[37] L.A. Zadeh, Fuzzy sets, Information and Control, Vol.8 (1965) N.3, 338-353.

[38] Bei Zeng, Xie Chen, Duan-Lu Zhou, Xiao-Gang Wen, Quantum Information Meets Quantum Matter, arXiv:1508.02595v4.

California Institute of Technology, USA

Perimeter Institute for Theoretical Physics, Canada

University of Toronto, CANADA

E-mail address: matilde@caltech.edu

E-mail address: matilde@math.utoronto.ca

E-mail address: mmarcolli@perimeterinstitute.ca 